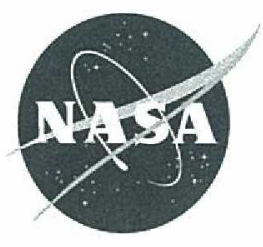

\title{
Accurate Measurements of the Dielectric Constant of Seawater at L Band
}

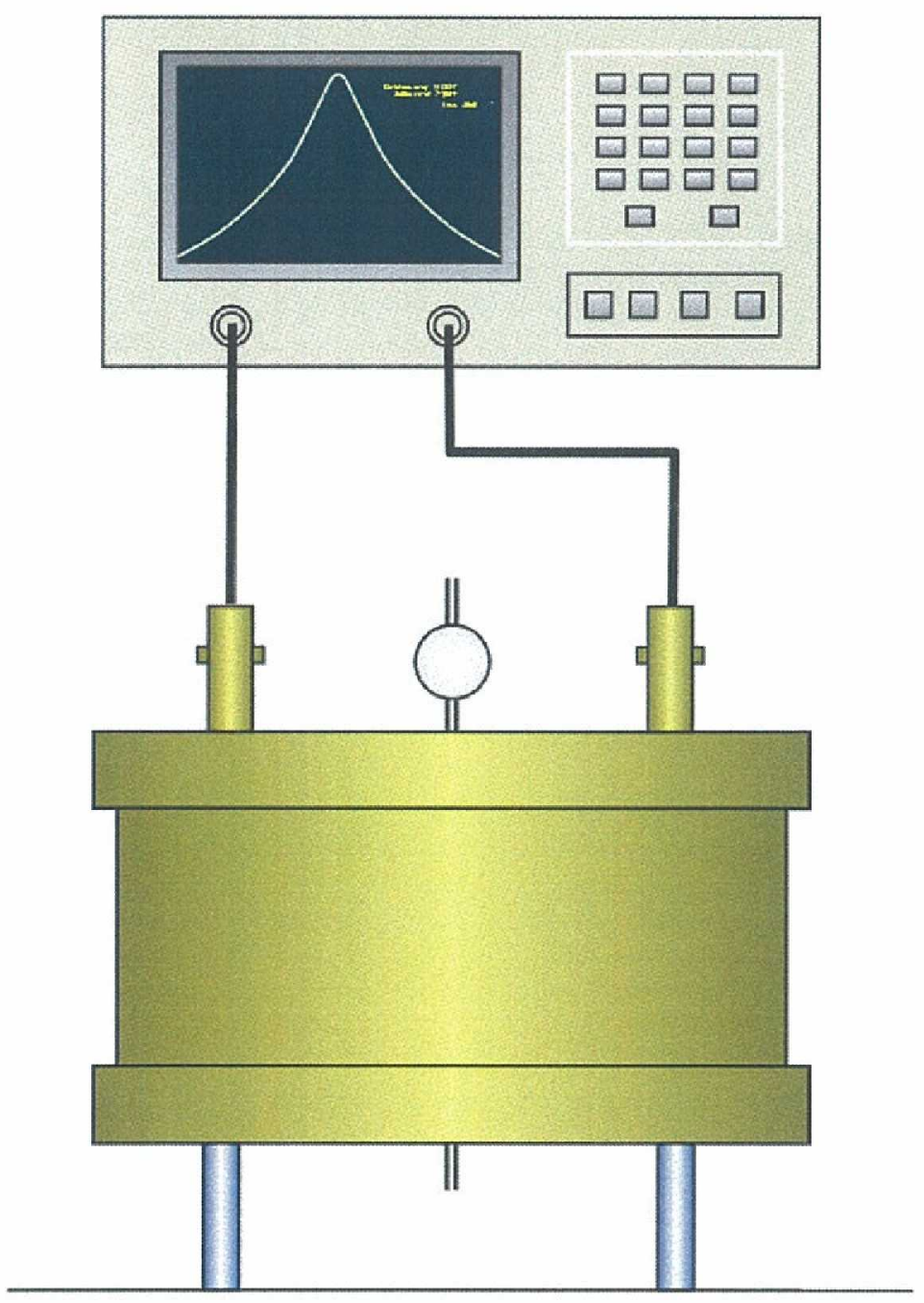


The NASA STI Program Office ... in Profile

Since its founding, NASA has been dedicated to the advancement of aeronautics and space science. The NASA Scientific and Technical Information (STI) Program Office plays a key part in helping NASA maintain this important role.

The NASA STI Program Office is operated by Langley Research Center, the lead center for NASA's scientific and technical information. The NASA STI Program Office provides access to the NASA STI Database, the largest collection of aeronautical and space science STI in the world. The Program Office is also NASA's institutional mechanism for disseminating the results of its research and development activities. These results are published by NASA in the NASA STI Report Series, which includes the following report types:

- TECHNICAL PUBLICATION. Reports of completed research or a major significant phase of research that present the results of NASA programs and include extensive data or theoretical analysis. Includes compilations of significant scientific and technical data and information deemed to be of continuing reference value. NASA's counterpart of peer-reviewed formal professional papers but has less stringent limitations on manuscript length and extent of graphic presentations.

- TECHNICAL MEMORANDUM. Scientific and technical findings that are preliminary or of specialized interest, e.g., quick release reports, working papers, and bibliographies that contain minimal annotation. Does not contain extensive analysis.

- CONTRACTOR REPORT. Scientific and technical findings by NASA-sponsored contractors and grantees.
- CONFERENCE PUBLICATION. Collected papers from scientific and technical conferences, symposia, seminars, or other meetings sponsored or cosponsored by NASA.

- SPECIAL PUBLICATION. Scientific, technical, or historical information from NASA programs, projects, and mission, often concerned with subjects having substantial public interest.

- TECHNICAL TRANSLATION. English-language translations of foreign scientific and technical material pertinent to NASA's mission.

Specialized services that complement the STI Program Office's diverse offerings include creating custom thesauri, building customized databases, organizing and publishing research results ... . even providing videos.

For more information about the NASA STI Program Office, see the following:

- Access the NASA STI Program Home Page at http://www.sti.nasa.gov/STI-homepage.html

- E-mail your question via the Internet to help@sti.nasa.gov

- Fax your question to the NASA Access Help Desk at (443) $757-5803$

- Telephone the NASA Access Help Desk at (443) 757-5802

- Write to: NASA Access Help Desk NASA Center for AeroSpace Information 7115 Standard Drive Hanover, MD 21076 


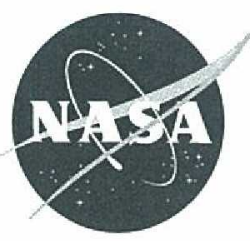

\section{Accurate Measurements of the Dielectric Constant of Seawater at $L$ Band}

\section{Final Report}

Roger H. Lang, Principal Investigator

Department of Electrical and Computer Engineering

The George Washington University Washington, DC

Cuneyt Utku

NASA Goddard Space Flight Center Greenbelt, MD

Yalcin Tarkocin

Tritek Solutions, Annandale, VA

David M. Le Vine, Co-Investigator

NASA Goddard Space Flight Center Greenbelt, $M D$

Prepared under NASA Grant NNG-05GO48G

August 1, 2005 to August 31, 2009

Prepared for Dr. Eric Lindstrom

NASA Headquarters, Code SMD

Washington, DC 20546

National Aeronautics and

Space Administration

Goddard Space Flight Center

Greenbelt, Maryland 20771 
Trade names and trademarks are used in this report for identification only.

Their usage does not constitute an official endorsement, either expressed or implied, by the National Aeronautics and Space Administration.

Available from:

NASA Center for AeroSpace Information

7115 Standard Drive

Hanover, MD 21076-1320
National Technical Information Service 5285 Port Royal Road Springfield, VA 22161 


\section{Table of Contents}

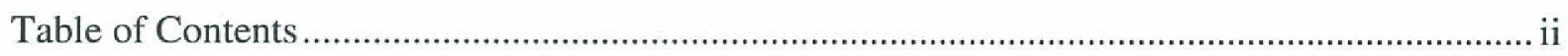

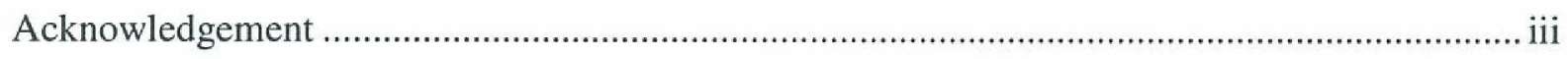

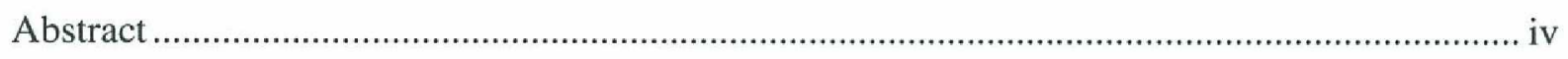

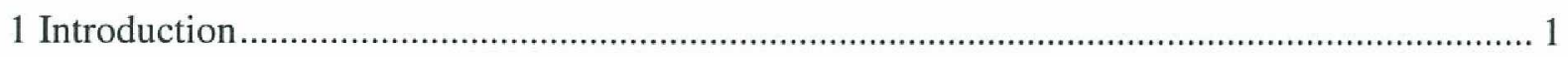

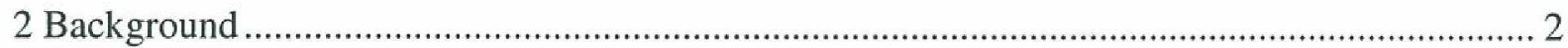

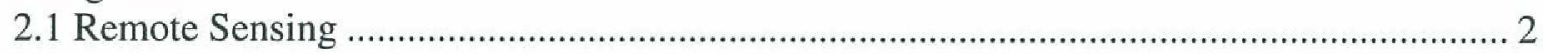

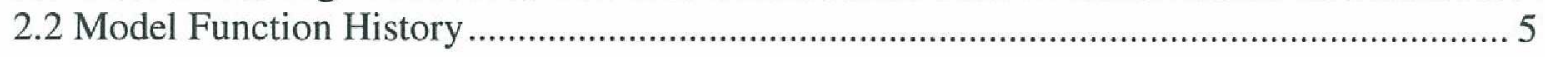

3 Measurement of the Dielectric Constant of Seawater............................................................. 6

3.1 General Approach ....................................................................................................... 7

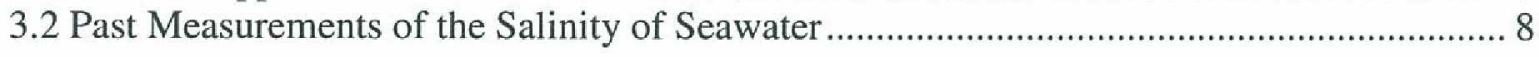

4 Measurements at The George Washington University ............................................................ 10

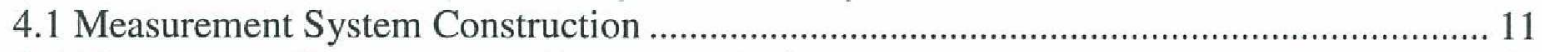

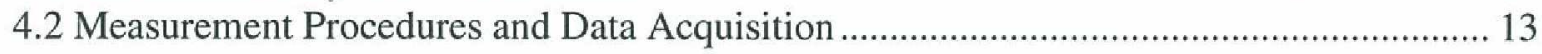

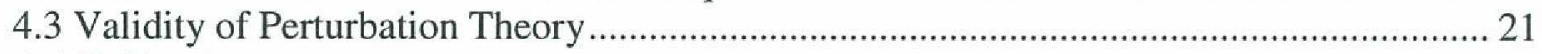

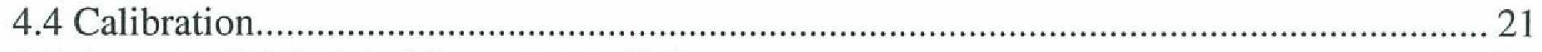

4.5 Seawater Dielectric Measurement Data....................................................................... 25

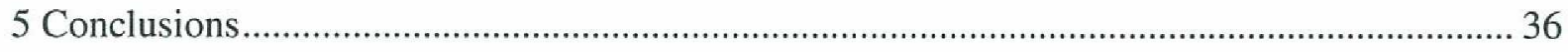

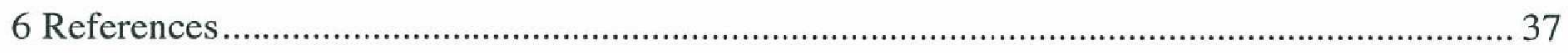

Appendix A Sensitivity of Salinity to Variations in the Model Function .................................. 41

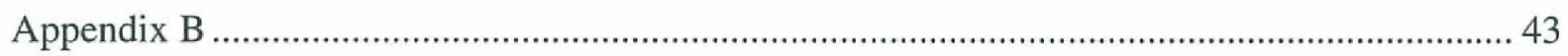

Derivation of the Perturbation Equations for the Measurement of ....................................... 43

Dielectric Constants ...................................................................................................... 43

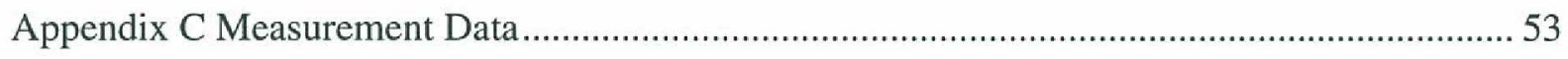

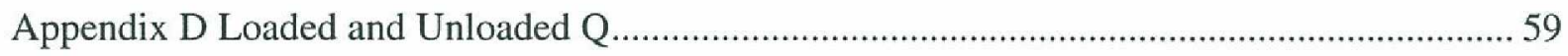




\section{Acknowledgement}

The authors would like to thank Professor Jerzy Krupka of the Warsaw University of Technology in Warsaw, Poland for helping with the initial design of the cavity and Drs. James Baker-Jarvis and Dr. Michael Janezic of National Institute of Standards and Technology (NIST) for the advice that they have given. We would like to thank Professor Thomas Manuccia who advised Jared Janiczek in the construction of the control and automation systems for his senior project at The George Washington University (GW). In addition, we would like to thank Hirad Ghaemi, Shuyi Wang, Yi Jin and Shuo Gu for helping with measurements and data analysis. Finally, we would like to thank Dr. Allan Schneider for his detailed reading of the report. 


\begin{abstract}
This report describes measurements of the dielectric constant of seawater at a frequency of 1.413 $\mathrm{GHz}$ that is at the center of the L-Band radiometric protected frequency spectrum. Aquarius will be sensing the sea surface salinity from space in this band. The objective of the project is to refine the model function for the dielectric constant as a function of salinity and temperature so that remote sensing measurements can be made with the accuracy needed to meet the measurement goals ( $0.2 \mathrm{psu})$ of the Aquarius mission.

The measurements were made, using a microwave cavity operated in the transmission configuration. The cavity's temperature was accurately regulated to $0.02^{\circ} \mathrm{C}$ by immersing it in a temperature controlled bath of distilled water and ethanol glycol. Seawater had been purchased from Ocean Scientific International Limited (OSIL) at salinities of 30, 35 and 38 psu. Measurements of these seawater samples were then made over a range of temperatures, from $10^{\circ} \mathrm{C}$ to $35^{\circ} \mathrm{C}$ in $5^{\circ} \mathrm{C}$ intervals. Repeated measurements were made at each temperature and salinity. Mean values and standard deviations were then computed. Total error budgets indicated that the real and imaginary parts of the dielectric constant had a relative accuracy of about $1 \%$. Comparisons with the model function of Klein and Swift showed that the real part of the dielectric constant was about $1.5 \%$ below their values while the imaginary part, on the average, was about $2 \%$ below the Klein and Swift results. The differences in the imaginary parts between the two results increased with rising temperature and salinity.
\end{abstract}




\section{Introduction}

This report describes $\mathrm{L}$ band measurements of the dielectric constant of seawater using a resonant cavity approach. The measurements have been made in support of the space-borne Aquarius mission with its goal of measuring sea surface salinity (SSS) to an accuracy of 0.2 psu. Because of the small variation in sea surface salinity over the open ocean, highly accurate L band radiometric measurements are required to sense these variations. Sensitivity calculations show that in order to achieve the required accuracy of 0.2 psu for sea surface salinity measurements, the brightness temperature measured by a satellite radiometer must be accurate to $0.1^{\circ} \mathrm{K}$. This requirement, in turn, places an accuracy requirement of $0.2 \%$ on the dielectric constant measurements used in the retrieval (i.e., the construction of the model function, $\varepsilon(T, S)$ ). It is not clear that existing model functions such as Klein and Swift (1977) satisfy these accuracy requirements. The Klein and Swift model function, for example, is based, in part, on the dielectric measurement data of Ho and Hall (1973) and Ho et al (1974). These measurements were made over thirty years ago without the advantages of modern test equipment. In addition, they did not adequately cover the range of temperatures and salinity of interest to Aquarius mission.

Salinity measurements made before 1978 were expressed in parts per thousand (\%o) while after 1978 the measurements were expressed in practical salinity units (psu). The model function of Klein and Swift was based on measurements made in parts per thousand. The new measurements, presented in this report, were made in practical salinity units. Comparison of the data presented in this report with the Klein and Swift model function will not be a problem, however. Lewis and Perkins (1981) point out that the two scales differ by less than .001 in the salinity range, $25<\mathrm{S}<38$ for salinities computed from chlorinity using the Knudsen relationship (Forch, et al 1902). Since the Ho et al (1974) measurements were made in parts per thousand using the Knudsen relationship, measurements made in parts per thousand and in practical salinity units can be used interchangeably with only negligible error.

The dielectric constant measurements of seawater described in this report have been made at $1.413 \mathrm{GHz}$. This is approximately the center frequency of the quiet zone (radiometric protected band) from $1.400 \mathrm{GHz}$ to $1.427 \mathrm{GHz}$ that Aquarius will utilize when making its measurements. The measurements have been made at temperatures of $10^{\circ} \mathrm{C}$ to $35^{\circ} \mathrm{C}$ in $5^{\circ}$ increments with salinity values of 30,35 and 38 psu. Although the range of the measurement set should be extended, these measurements represent values characteristic of open ocean conditions - the primary objective of the Aquarius mission.

In this report a time dependence of $\exp (-i \omega t)$ will be assumed. This assumed time dependence for Maxwell's time harmonic equations leads to the expression of the complex relative dielectric constant as $\varepsilon=\varepsilon^{\prime}+i \varepsilon^{\prime \prime}$ where $\varepsilon^{\prime}$ and $\varepsilon^{\prime \prime}$ are the real and imaginary parts of the relative dielectric constant respectively. For lossless or lossy media, $\varepsilon^{\prime \prime}$ will be greater or equal to zero $\left(\varepsilon^{\prime \prime} \geq 0\right)$.

The report is structured as follows: in Section 2, sensitivity requirements for the dielectric constant measurements of seawater are derived based on the measurement accuracy goal of 0.2 psu. In the same section, a review of past measurement data for this frequency band will be 
made. In addition, the use of out of band data to estimate in-band dielectric constants using model functions will be presented. In Section 3, a review of the available methods for the accurate measurement of the dielectric constant of lossy liquids is given, along with a detailed discussion of how past measurements have been made. In Section 4 the resonant cavity approach used by The George Washington University (GW) group is described along with the measurement data and the method used for calibration of the cavity. Finally in Section 5, conclusions and future work are discussed.

\section{Background}

In the following two subsections, there is presented a brief discussion of how the brightness temperature - measured over a half space of seawater - varies with temperature and salinity. In this context, the sensitivity of salinity to errors in the complex dielectric constant of seawater is then explored. This analysis is followed by a survey of seawater model function development over the last 40 years.

\subsection{Remote Sensing}

In remote sensing of sea surface salinity, the observed brightness temperature is directly related to the dielectric constant of the sea surface. The dielectric constant of the seawater is then related to the salinity, $S$, and the temperature, $T$, of the seawater by the model function, $\varepsilon(T, S)$. These relationships are illustrated in eq(1) for the case of a radiometer looking directly down, normal to a flat sea surface (the simplest case). Here the brightness temperature, $T_{B}$, is given by

$$
T_{B}=\left(1-|R|^{2}\right) T
$$

where $R$ is the Fresnel reflection of the surface, that is,

$$
R=(1-\sqrt{\varepsilon}) /(1+\sqrt{\varepsilon}) .
$$

The relative dielectric constant of the seawater, $\varepsilon$, is given in terms of the model function:

$$
\varepsilon=\varepsilon(T, S)
$$

The dielectric constant contains the electrical properties of the medium and it is here that the dependence on salinity appears.

As an illustration, eq(1a) is shown in Figure 1 using the model function developed by Klein and Swift (1977). The radiometer output, $T_{B}$, is plotted on the vertical axis versus the seawater temperature, $T$, on the abscissa with the seawater salinity, $S$, as a parameter. The Klein and Swift model function has been the common choice for $\varepsilon(T, S)$ in recent remote sensing studies (Le Vine et al 1998, Goodberlet et al 1997, Lagerloef 1995). In the remote sensing paradigm, we measure $T_{B}$ and obtain $T$ from an ancillary source. The salinity is then found from Figure 1, at the intersection of $T_{B}$ and the seawater temperature $T$. Given the correct values of $T_{B}$ and $T$, the accuracy of the inversion is limited by the accuracy of the model function, $\varepsilon(T, S)$. 


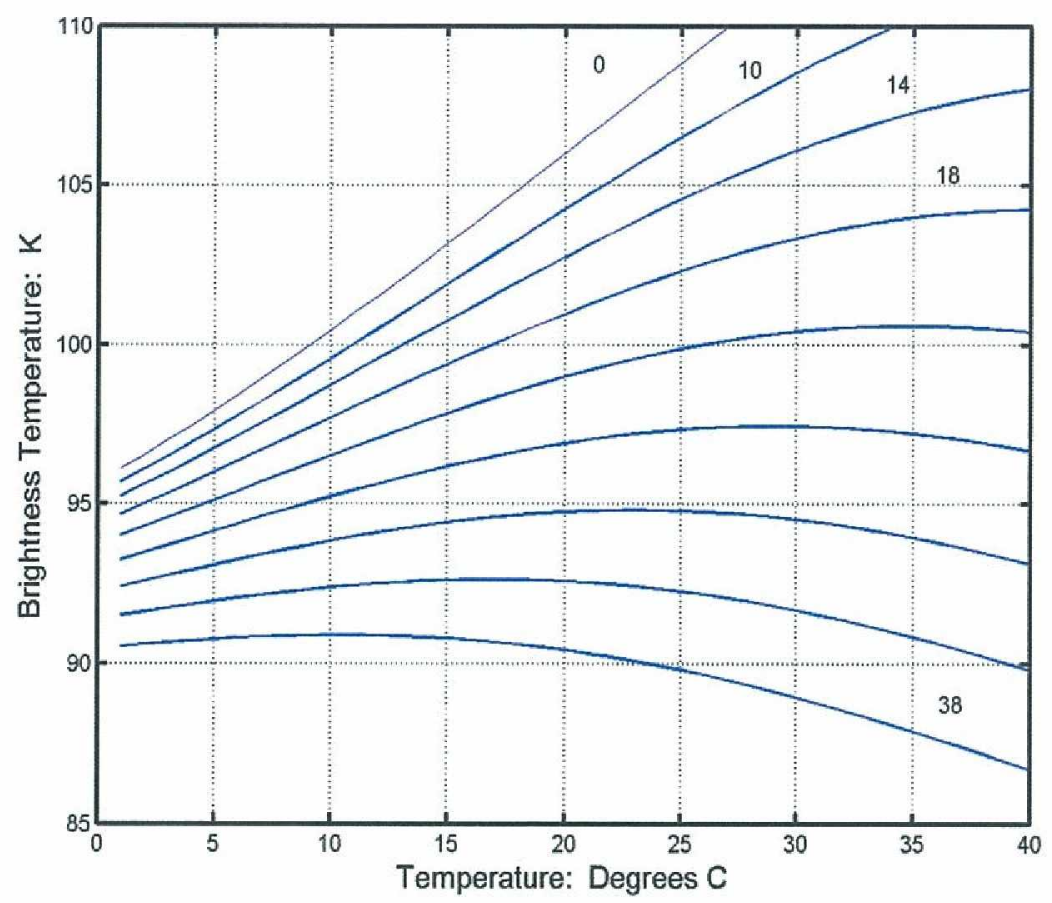

Figure 1 Brightness Temperature vs. Temperature with Salinity as a parameter

To understand the sensitivity of the seawater salinity inversion to errors in the model function, we examine the inversion in more detail. Let us assume that the exact model function is denoted by $\varepsilon(T, S)$ and the model function constructed from measurements is given by $\varepsilon_{m}(T, S)$. The error in the model function is: $\delta \varepsilon=\varepsilon-\varepsilon_{n n}$.

In performing the inversion, it is assumed that the brightness temperature, $T_{B}$, and the sea surface temperature, T, are known exactly. The model function is then used to find the salinity, $S$. To examine the sensitivity of the algorithm to changes in the model function, we determine the change in salinity, $\delta S$, for a given change in the model function, $\delta \varepsilon$. It should be noted that the dielectric constant is complex and thus $\delta \varepsilon$ is complex. The results of this calculation are shown in Figure 2. Here we have assumed that the measured model function is the Klein and Swift model; we have also assumed that the magnitude of $\varepsilon\left(|\varepsilon|=\sqrt{\varepsilon^{\prime 2}+\varepsilon^{\prime \prime 2}}\right)$ is large (a good approximation for seawater at $1.413 \mathrm{GHz}$ ).

Figure 2(a) shows the impact of a 0.2 change in the real and the imaginary parts of the model function on the change in the salinity. It illustrates that the error in retrieved salinity can be larger than $0.2 \mathrm{psu}$ (the goal for the Aquarius mission) and is larger in cold water than in warm water. This should not be surprising since an examination of Figure 1 shows that the constant salinity curves come closer together (decreased radiometric sensitivity) as the temperature decreases. This fact has been pointed out by Dinnat et al (2003). Figures 2(b) and 2(c) show the same curves as in Figure 2(a) except now the change in dielectric constant is completely in the 
(a)

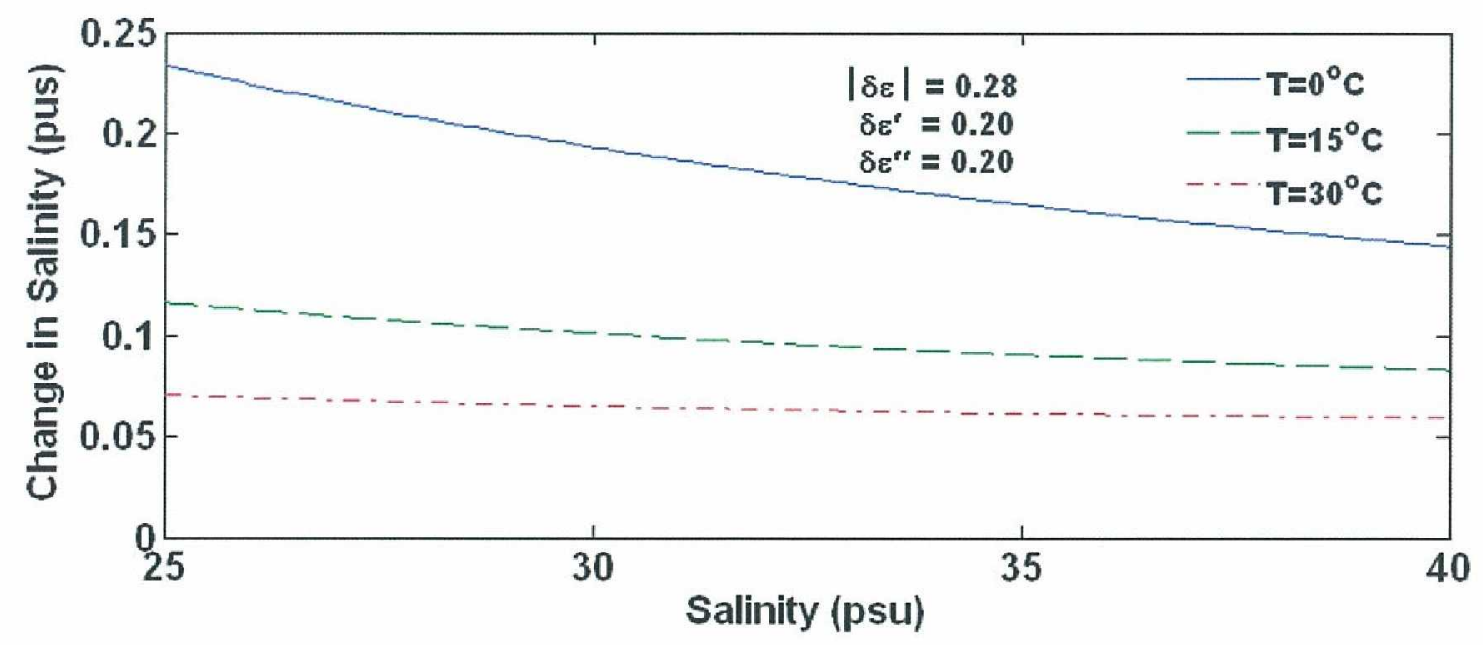

(b)

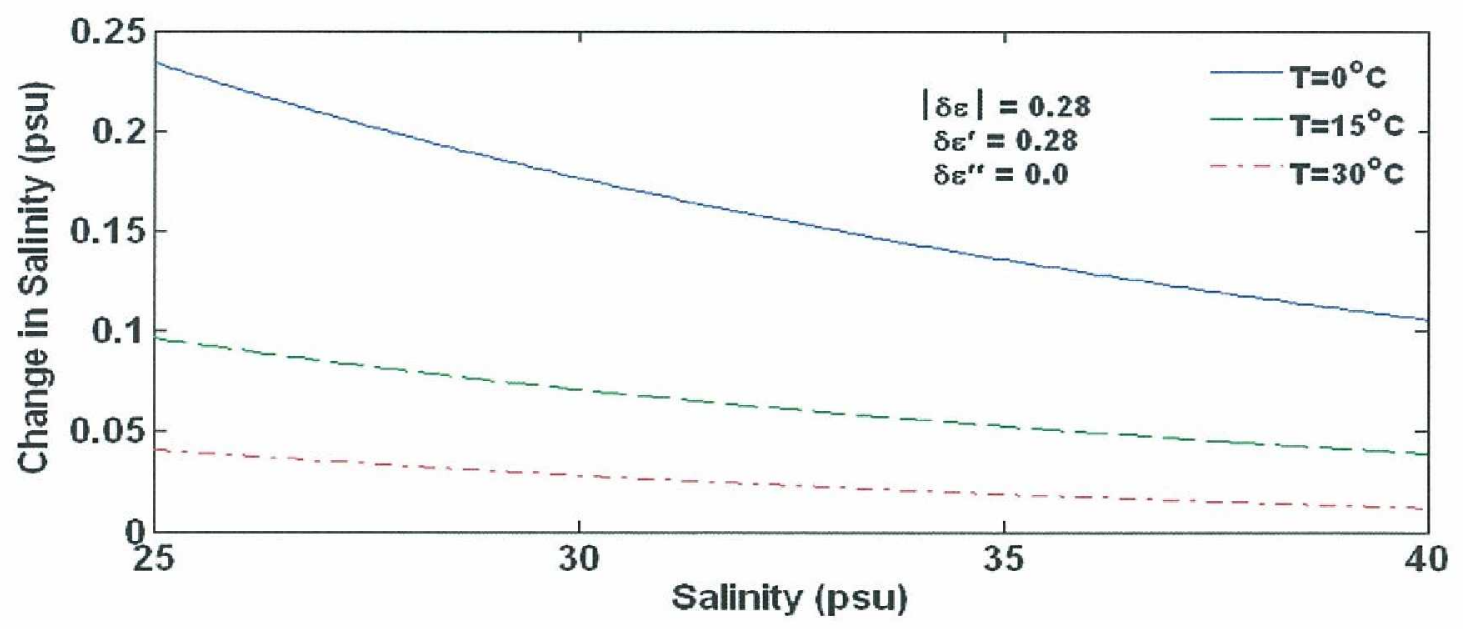

(c)

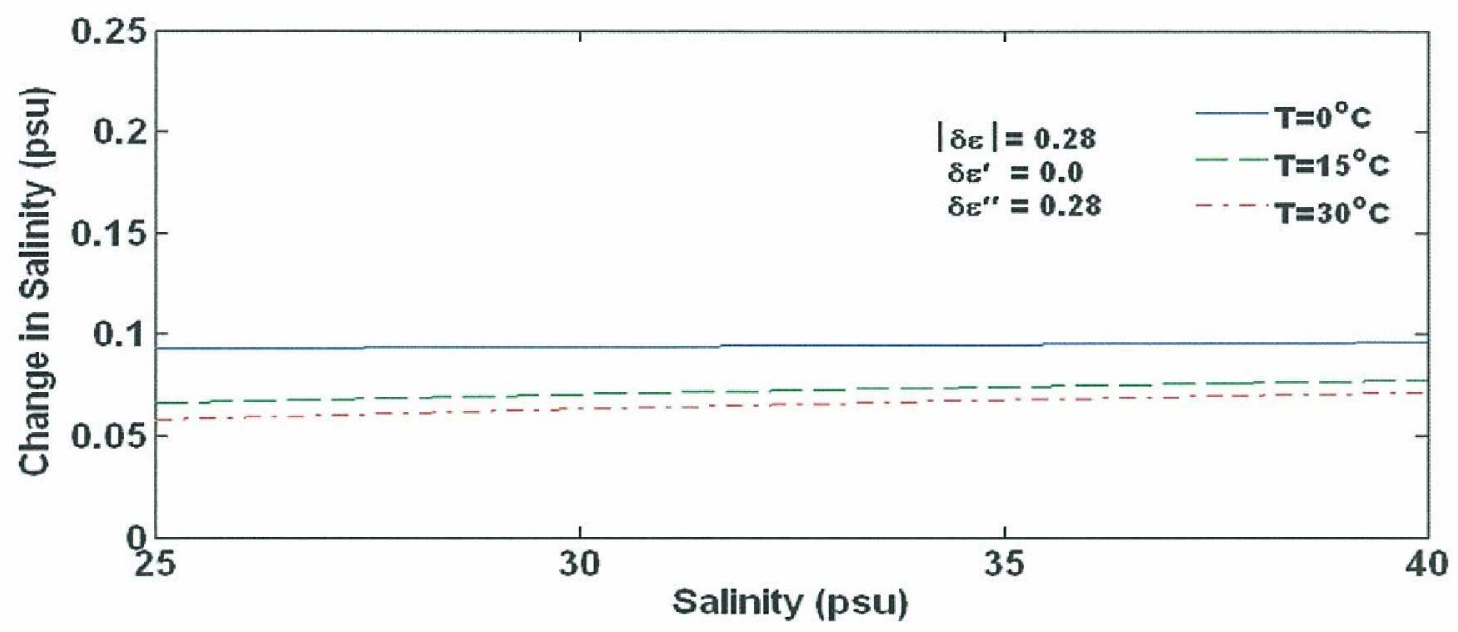

Figure 2. Change in Salinity versus Salinity for several temperatures. In (a), (b) and (c) all errors have the same magnitude but different real and imaginary parts. 
real part (b) or in the imaginary part (c). The interesting feature is the effect of changing only the imaginary part is much smaller than changing the real part. This says that the real part must be measured more accurately than the imaginary part. The details of the sensitivity calculation, used to produce these curves, are given in Appendix A.

\subsection{Model Function History}

The model function used in Figure 1 was developed more than 25 years ago by Klein and Swift (1977). They used measurements in the frequency range $1-3 \mathrm{GHz}$ that were made by Ho and Hall (1973) and Ho et al (1974). These measurements were used to refine the existing parameterization for the dependence of $\varepsilon$ on $T$ and $S$ at L- band. This parameterization was based on the Debye model for the dielectric constant proposed by Stogryn (1971). The result was a set of regression curves for these coefficients as a function of $T$ and $S$ that have served the remote sensing community well since 1977 and have been used in much of the research demonstrating the potential for passive remote sensing of salinity (Blume et al 1978, Goodberlet et al 1997, Le Vine et al 1998). Much of this work, however, has been on salinity fields with large gradients, such as those occurring in the coastal environment where measurement accuracy of 1 psu is adequate.

The goal of modern remote sensing of the global salinity field from space is almost an order of magnitude more ambitious. For example, the goal of Aquarius is 0.2 psu (Koblinsky et al 2003) and the goal of SMOS is 0.1 psu (Sivestrin et al 2001). Achieving such accuracy requires careful control of all elements of the remote sensing system. This control would include, for example, a well designed radiometer that gives accurate values of $T_{B}$, gives good sources of ancillary data such as Sea Surface Temperature and also gives an accurate accounting of environmental factors such as emission and attenuation along the propagation path. Modern measurements (Stogryn et al 1995, Ellison et al 1998, Meissner and Wentz 2004, Blanch and Agusca 2004; Lang et al $2008 \mathrm{a}, \mathrm{b})$ suggest that refinements are also needed in the model function. They point out that certain aspects of the Ho data may be in error and, as a result, the Klein and Swift model function may need modification. The recent measurements of Blanch and Aguasca (2004) indicate differences when compared to the Klein and Swift (1977) model function. If valid, these differences will result in errors larger than the 0.1-0.2 psu goal of Aquarius and SMOS. Stogryn et al (1995) has suggested an alternate parameterization of the dependence of conductivity on salinity and temperature. At L-band, the influence of salinity is primarily through its effect on conductivity. The modern definition of salinity, in fact, is based on the measurement of conductivity (Lewis, 1980).

Modern measurements have also been made at higher frequencies (Stogryn 1995, Ellison et al. 1998, Meissner and Wentz 2004). These measurements also suggest that changes in the model function may be needed. Figure 3 shows a comparison of the Ellison et al model function with the Klein and Swift model function. The two, based on data in different frequency ranges, employ regression methods to fit the salinity and temperature dependence. At first glance, the agreement is good (because both employ the Debye model). The scale in Figure 3, however, is very large and the differences that appear modest in Figure 3 are far too large to achieve an accuracy of 0.2 psu.

Another example is shown in Figure 12 of section 4.5.2, where measurements made at GW are 

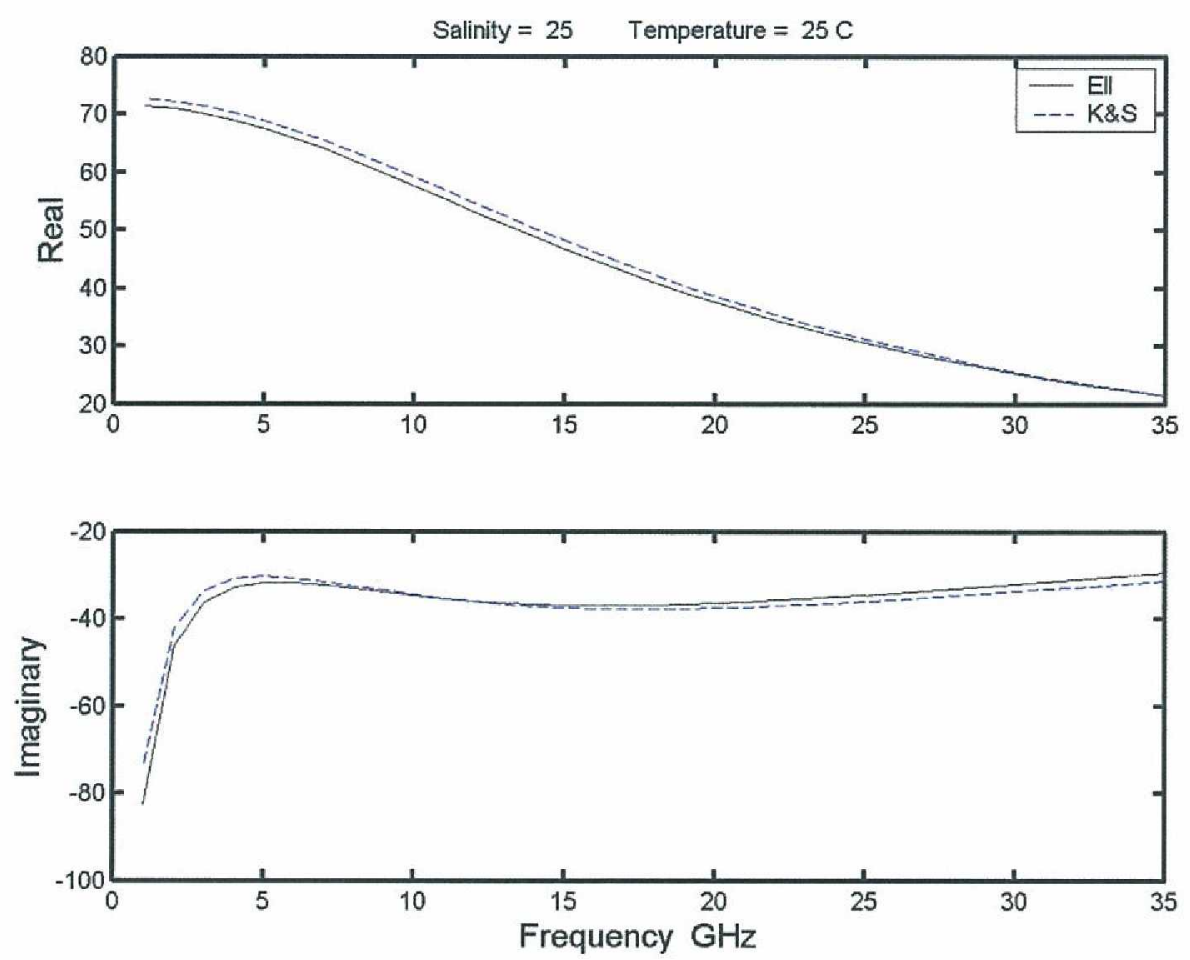

Figure 3. Seawater Permittivity from Ellison and Klein and Swift

plotted together with the Klein and switt model for $s=3 U$ psu. Although the trend with temperature is in agreement with the Klein and Swift model, there is clearly an offset (bias), especially apparent in the real part of the dielectric constant. The bias is significant for a remote sensing goal of 0.2 psu. Similar behavior is seen when comparing the Blanch and Aguasca measurements with the Klein and Swift model function.

The goal of the research work reported here is to make accurate seawater measurements for a range of temperatures and salinities characteristic of the open ocean at a frequency of $1.413 \mathrm{GHz}$ that is in the center of the Aquarius frequency band. Each measurement at a specific temperature and salinity is repeated several times; the mean and variance are also reported. Before describing the measurements in section 4, additional details of the background and measurement technique are discussed in Section 3. Finally, our conclusions are given in section 5 along with suggestions for future work.

\section{Measurement of the Dielectric Constant of Seawater}

In this section, various methods for making measurements of the dielectric constant of seawater are described. These descriptions are then followed by a discussion of the results of past measurements of seawater. In Section 3.1, a review of the general approaches to the measurement process is given along with a discussion of main measurement devices presently in use. In Section 3.2, the measurement of the seawater dielectric constant by non GW investigators in the frequency band 1400 to $1430 \mathrm{MHz}$ is discussed. Measurements of the dielectric constant of seawater at other microwave frequencies will also be reviewed. 


\subsection{General Approach}

The need to know the dielectric constant of solids and liquids at microwave frequencies developed with the growth of radar during World War II. The results of this rapid progress have been documented by Montgomery (1947) in his chapter on dielectric measurements. Six generic measurement methods have evolved:

- free space reflection and transmission measurements

- waveguide transmission and reflection measurements

- reflection and transmission resonant cavity measurements

These measurement methods are still in use today. Improvements in microwave instruments and components however, make today's measurement results much more accurate .

The dielectric constant of liquids has been measured by all six methods, although the need to confine liquids puts some constraints on the physical configuration. The first method is the free space reflection technique that has been employed by Saxton and Lane (1952) and more recently by Blue (1980). The method has found use at mm wavelengths. Buckmaster (1990) modified the waveguide transmission approach of Nicolson and Ross (1970) to make dielectric constant measurements of high loss liquids below $10 \mathrm{GHz}$. Jenkins et al (1990) have employed a TEM coaxial transmission line shorted at one end to make measurements of the dielectric constant of several alcohols by the reflection technique. This is a modern version of a reflection technique developed by Roberts and von Hippel (1946). Gregory and Clark (2001, 2009) have used coaxial transmission and reflection cells to make very accurate measurements of alcohols from 0.1 to $5.0 \mathrm{GHz}$.

In all of the methods except the resonant cavity approach, the frequency can be swept to cover a range of frequencies. The resonant cavity method, on the other hand, is usually a single frequency measurement. This method, however, is considered the most accurate of the techniques (Baker-Jarvis, et al 1998, Baker-Jarvis, et al 2001). There are two types of cavity resonators: reflection and transmission. The reflection cavity only has one port and $\mathrm{S}_{11}$ is measured; the transmission cavity has two ports and $\mathrm{S}_{21}$ is measured. The reflection cavity approach is the method that has been used by Ho and Hall (1973) and Ho et al (1974) to measure the dielectric constant of seawater. Dielectric resonators can also be used to measure seawater; however, their Q may not be high enough (Krupka, 1988).

Another important advantage of the resonant cavity approach, when making highly accurate measurements, is that the seawater can be isolated within the cavity via a glass tube. In waveguide transmission and reflection cells, the seawater is in direct contact with the metal walls of the cell. Because of the corrosive character of seawater, its interaction with the metal walls may lead to serious problems. Stogryn (1995) reported difficulties with repeatability due to this problem. To minimize the effects, Stogryn gold-plated the waveguide and washed the walls with hydrochloric acid after each measurement. He also mentioned that if the seawater were to remain in the transmission cell too long, its salinity would change. 


\subsection{Past Measurements of the Salinity of Seawater}

Over the years, there have been relatively few microwave and mm wave measurements of the seawater dielectric constant. Saxton and Lane (1952) conducted one of the earliest investigations. They measured the dielectric constant of an $\mathrm{NaCl}$ solution that had properties close to those of seawater (Ho et al 1974) for frequencies between 9.35 and $48.2 \mathrm{GHz}$ by the surface reflection technique. The first measurements of actual seawater were made by Ho and Hall (1973) using a reflection cavity technique at $2.65 \mathrm{GHz}$. This was followed by Ho et al (1974) who reported on seawater measurements at $1.43 \mathrm{GHz}$ - again using a reflection cavity technique. Blue (1980) made measurements at $97,103.8,135.6$ and $183.3 \mathrm{GHz}$ using the free space reflection method. Stogryn (1995) made measurements of seawater from $7 \mathrm{GHz}$ to $12 \mathrm{GHz}$ by using a waveguide transmission cell. Ellison (1998) made measurements from 30 to $105 \mathrm{GHz}$ employing a coaxial transmission cell at the lower frequencies and a free space transmission cell at the higher frequencies. In 2004 Blanch and Aguasca measured the seawater dielectric constant over a range of $\mathrm{L}$ band frequencies using a microline transmission cell. In the same year Meissner and Wentz (2004) used SSMI satellite measurements to determine, indirectly, the sea surface dielectric constant at the SSMI frequencies. Most recently, Lang et al (2008a,b) reported transmission cavity measurements made at $1.413 \mathrm{GHz}$.

For the measurement of ocean salinity, we are concerned mainly with measurements made in the window of 1.400 to $1.430 \mathrm{GHz}$. These include the measurements of Ho et al (1974), Blanch and Aguasca (2004) and Lang et al (2008a,b). The other works, however, contain a great deal of useful information on measurement techniques. Stogryn et al (1995) in particular conducted very accurate measurements by driving down the fluctuations with repeated measurements.

\subsubsection{Measurements made by Ho et al (1974)}

Ho et al (1974) employed the resonant cavity approach to measure the dielectric constant of seawater at $1.43 \mathrm{GHz}$. They used the resonant cavity approach because of its known accuracy and because the permittivity was needed at only one frequency. They built a cylindrical cavity out of copper that was then excited in the $\mathrm{TM}_{010}$ mode. The liquid was then introduced into the cavity through a coaxial glass tube with a very small inner diameter. The resulting microwave signal was coupled into the cavity by a single loop (reflection cavity approach). The reflection coefficient was then measured at the resonant frequency and at some off-resonance frequency such as the frequency of the half power point.

Under the assumption that the amount of seawater introduced into the cavity is small, a perturbation theory (Chen et al 2004) can be applied to obtain the following formulas:

$$
\varepsilon^{\prime}-1=2 C \frac{\Delta f}{f}, \quad \varepsilon^{\prime \prime}=C \Delta(1 / Q)
$$

where

$$
\Delta f=f_{0}-f, \quad \Delta(1 / Q)=\frac{1}{Q}-\frac{1}{Q_{0}}
$$


Here $\varepsilon^{\prime}$ and $\varepsilon^{\prime \prime}$ are the real and the imaginary parts of the dielectric constant of the sample respectively. The quantities $f_{0}$ and $Q_{0}$ are, respectively, the resonant frequency of the cavity and its Q-factor when there is no sample in the cavity. The same quantities without the subscripts are the values of the resonant frequency and $\mathrm{Q}$ with a sample in the cavity. A derivation of these formulas is presented in Appendix B that accounts for cavity wall loss and gives a means of computing the error made when using eq(2).

The quantity $C$ appearing in eq(2a) is called the calibration constant. It can be evaluated by two methods: relative and absolute (Ellison 1996). When using the relative technique, another liquid of known dielectric constant is measured in the cavity. Since $\varepsilon^{\prime}$ and $\varepsilon^{\prime \prime}$ are known and $\Delta f$ and $\Delta(1 / Q)$ are measured, the constant $C$ can be determined from eq(2a). The quantity $C$ can also be obtained from the expressions for the electromagnetic fields in the cavity. This is known as the absolute calibration technique. It becomes increasingly more accurate as the cavity is modeled in more detail. From perturbation theory (Chen et al 2004) it is shown that the calibration coefficient appearing in eq(2a) is inversely proportional to the volume of the tube holding the sample. For example, a $1 \%$ error in tube volume results in a measured dielectric constant having a one percent error.

Ho et al (1974) used both the relative and absolute methods in making their measurements of seawater. They employed the relative technique to make the actual seawater measurements. They used distilled water as a reference. At that time, however, there was relatively little distilled water dielectric data available near their measurement frequency. As a result, they used their cavity to measure the dielectric constant of distilled water (absolute method) and used expressions for the electromagnetic fields in the cavity to obtain an expression for the calibration coefficient. They then carefully measured the volume of the sample in the cavity by two techniques. Their findings for the dielectric constant of distilled water have not been checked by using another measurement approach at $1.43 \mathrm{GHz}$.

Ho et al (1974) claimed an accuracy of $0.2 \%$ for their measurement of $\varepsilon^{\prime}$ and $0.4 \%$ for their measurement of $\varepsilon^{\prime \prime}$ for seawater at $1.43 \mathrm{GHz}$. This is an average accuracy for values obtained for temperatures from $5^{\circ} \mathrm{C}$ to $30^{\circ} \mathrm{C}$ and for salinities from $5 \%$ to $35 \%_{0}^{* *}$. But these results assume that the dielectric constant of the reference liquid (distilled water) was known exactly. However, we have no way of knowing the accuracy of their measurement of distilled water by the absolute method (modeling the cavity accurately). They tried to compare their dielectric measurements of distilled water with available data but the available data was very sparse. In addition, their seawater measurements do not reduce to the distilled water dielectric values when these are extrapolated to zero salinity. Ho et al 1974 claimed that this was the effect of organic material in the seawater. Ellison (1996), however, has shown experimental results indicating that organic material in seawater has little effect on the dielectric constant. Another potential source of error is microwave heating of the seawater in the cavity. Swift (2006) mentioned that heating effects may have introduced some additional errors in the imaginary part of the dielectric

\footnotetext{
** Salinity measurements before 1978 will be reported in parts per thousand (\%o) while the measurements after 1978 will be given in psu. As pointed out in the introduction, the numbers can be used interchangeably in the range of salinities and temperatures we are considering.
} 
constant when the salinity and temperature are high. High salinity values (30\%o to 40\%o) are of the most interest in open ocean salinity measurements.

More recently Stogryn et al. (1995) made measurements of seawater from 7 to $14 \mathrm{GHz}$ by using a temperature controlled transmission waveguide of variable length. They measured seawater with a salinity range from 0 to $38 \mathrm{psu}$ with temperatures from $0^{\circ} \mathrm{C}$ to $30^{\circ} \mathrm{C}$. They proposed a Debye permittivity model with a double relaxation time to better fit the data at higher frequencies. In the process of determining the Debye parameters they combined their data ( 7 to $14 \mathrm{GHz}$ ) with the Ho and Hall (1973) data at $2.65 \mathrm{GHz}$ and with the Ho et al (1974) data at 1.43 GHz. They found the deviation from the Debye model for this data set was several percent. When they eliminated the Ho data, the error reduced to approximately $0.8 \%$. They conjectured that the Ho data contained systematic errors and speculated that these errors could be due to microwave heating of the sample within the quartz tube. They pointed out that this was a possibility because of the extremely small diameter of the tube.

In summary, although the Ho et al (1974) measurements were carried out very carefully, their accuracy is uncertain, and since the Klein and Swift model function is based in part on Ho's measurements, the Klein and Swift model function could be in error as well. The need for measurements with well documented accuracy to support modern remote sensing - as those measurements being performed by SMOS and about to be performed by Aquarius - has led a need for new measurements. These include the measurements in support of SMOS by Blanch and Aguasca (2004) described below.

\subsubsection{The measurements of Blanch and Aguasca (2004)}

Blanch and Aguasca (2004) have made measurements of the dielectric constant of seawater using a transmission cell. They constructed a transmission cell with a stripline structure through which seawater flows. Thermometers at the beginning and the end of the cell sensed the water temperature in the cell. A large quantity of seawater initially at $0^{\circ} \mathrm{C}$ was gradually heated as it flowed through the transmission cell. During this process, S-parameter measurements were continuously taken. The results obtained are close to the Klein and Swift model function for the real part of the permittivity. They are somewhat greater than the Klein and Swift model for the imaginary part by $1 \%$ to $2 \%$. The authors did not give many experimental details such as the accuracy of the temperature measurements and error bounds for their measured values. The goal of the GW measurements was to provide data with the accuracy needed to achieve the remote sensing goals of Aquarius and equally important to document the accuracy with error bars.

\section{Measurements at The George Washington University}

The measurement program at GW that has been supported by this grant began about 4 years ago with the goal of focusing specifically on the dielectric constant of seawater at frequencies relevant to the remote sensing of salinity,i.e., frequencies in the radio astronomy window at Lband centered at $1.413 \mathrm{GHz}$ ). A microwave cavity measurement system at $1.413 \mathrm{GHz}$ was constructed to measure the dielectric constant of seawater over values of temperature and salinity

characteristic of the open ocean. The emphasis focused on making the measurements as accurate as possible and on documenting error bars for the measurements and calibration. 


\subsection{Measurement System Construction}

After detailed consideration of many techniques available, it was decided that a resonant microwave cavity would yield the most accurate measurements given the fact that dielectric measurements were needed for only one narrow frequency band (1400 to $1427 \mathrm{MHz}$ ). Although measurements of alcohols by Gregory and Clark (2009) were made with high accuracy, the contact of the salt water with the metal walls for transmission and reflection cells could lead to problems. As pointed out previously, Stogryn et al (1995) reported difficulties in repeatability due to these problems. The use of a resonant cavity is similar to the method employed by Ho et al (1974) with one exception. Ho et al (1974) chose a reflection cavity having only one coupling loop to measure $S_{11}$. We have chosen a cavity of the transmission type that has two coupling loops and measures $S_{21}$. The reason for choosing a cavity of the transmission type is that muchless power is coupled into the cavity (Lang et al 2003). The heating problems that may have been observed in Ho's measurements will therefore not be a problem. In addition, transmission measurements are inherently more accurate than those of the reflection type. Ho et al (1974) used a cavity that resonated at $1430 \mathrm{MHz}$. This frequency is slightly outside of the quiet band. We have chosen a resonance frequency of $1413 \mathrm{MHz}$ that is in the center of the quiet band. If one uses the Klein and Swift model function to calculate dielectric constants, the difference in dielectric values for the two frequencies is less than $0.1 \%$.

A simplified drawing of the transmission resonant cavity setup is shown in Figure 4 . In the figure, a network analyzer (NA) is connected to a resonant cavity that is configured to measure

$\mathrm{S}_{21}$ over a band of frequencies about the cavity resonant frequency, $f_{o}$. The subscript, zero, indicates that the small glass sample tube shown in Figure 4 is empty. The blue curve generated

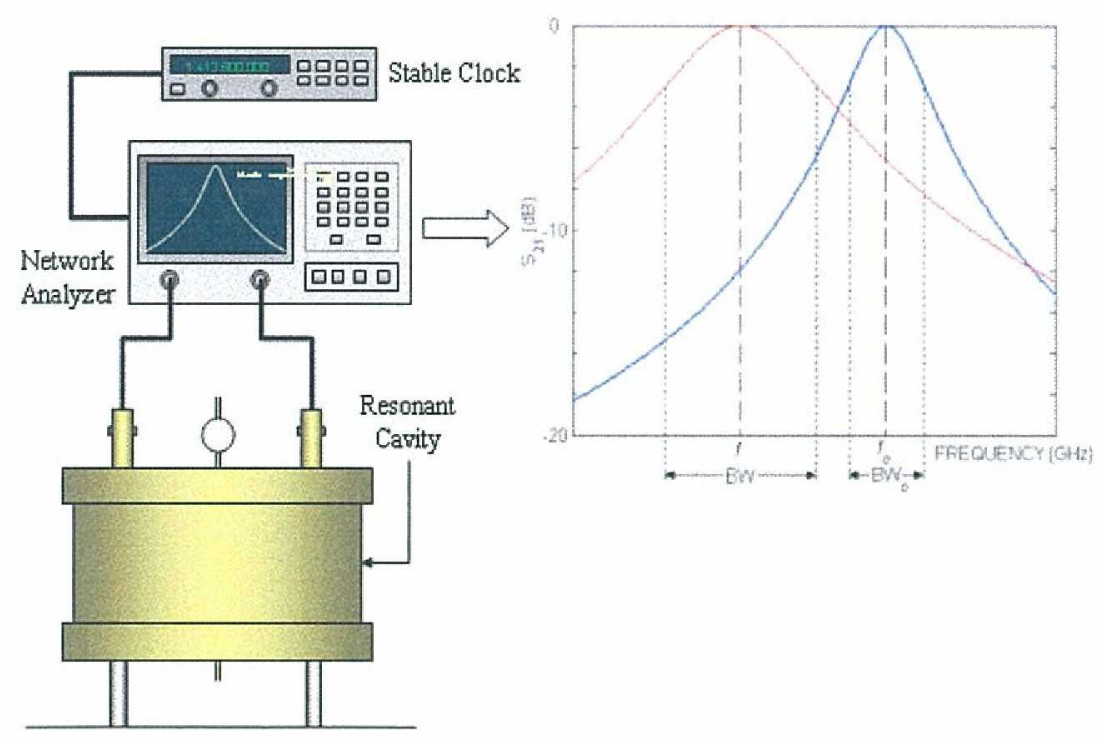

Figure 4. Resonant Microwave Cavity with Network Analyzer 
on the network analyzer screen and shown to the right of the NA in Figure 4 has a $3 \mathrm{db}$ bandwidth denoted by $\mathrm{BW}_{0}$. The $\mathrm{Q}$ of the cavity can be obtained from the $3 \mathrm{db}$ bandwidth by using the formula: $Q_{o}=f_{o} / \mathrm{BW}_{\mathrm{o}}$. Seawater is then put into the glass sample tube and the red curve is generated. Here the new resonant frequency is $f$ and the $Q=\mathrm{f} / \mathrm{BW}$. These quantities can be used in eqs (2a) and (2b) to determine the real and imaginary parts of the dielectric constant of the liquid in the sample tube. A small reservoir above the sample tube is used to bring the sample to the correct temperature before entering the tube.

The complete experimental setup is shown in Figure 5. In the figure, the resonant cavity is shown immersed in a liquid whose temperature is kept constant by the circulator shown on the left. The glass superstructure over the cavity is used as a conduit for nitrogen $\left(\mathrm{N}_{2}\right)$ to apply pressure on the seawater to force it into the sample tube. Also shown in this figure are the NA and the frequency stabilizer (counter). Detailed photographs of selected parts of the experimental apparatus are shown in Figure 6: the cavity with its glass superstructure appears in Figure $6 \mathrm{a}$, the inside view of the top cover of the cavity with its microwave coupling loops are shown in Figure $6 \mathrm{~b}$ and the glass sample tube that is placed along the axis of the cylindrical cavity and glass reservoir used to hold the seawater before entering the cavity are shown in Figure 6c.

The cavity that has been machined out of naval brass, has a Q of approximately 8000 . Brass has been used instead of copper since it can be machined more easily to greater precision. The cavity can be plated if higher conductivity walls are needed, but that has not been the case up to now. The seawater has been introduced into the cavity via a fused quartz tube of outer diameter of $3 \mathrm{~mm}$. The inner diameter (ID) of the sample tube was chosen to be $0.1 \mathrm{~mm}$. This is very small but is required if a reasonably large Q (about 4,000) is to be maintained when the seawater enters the cavity. This diameter also insures that the accuracy of the perturbation formulas given in equations (2) are within $0.1 \%$ as pointed out by Ho et al [1974] and demonstrated in Appendix B. Because the quartz tube ID is so small, the seawater sample will not usually enter the tube unless pressure is applied. To provide a means for the sample to enter the tube under pressure, a socket has been attached to the tube (see Figure 6c). Firing the quartz socket to quartz tube has proven to be a difficult task since heating the tube tends to close the small hole. The problem has been solved by forcing oxygen through the tube under pressure while the tube is fired to the socket. The top of the socket is attached to a reservoir to hold the sample. The reservoir has a small glass tube within it to hold a thermometer. This thermometer is used to measure the temperature of the sample. The reservoir is connected to a supply of dry industrial $\mathrm{N}_{2}$. Typically 2 atmospheres of $\mathrm{N}_{2}$ were used to push the sample through the tube once it reached the temperature of the surrounding liquid. The stable clock is used to keep the NA frequency correct to $1 \mathrm{~Hz}$.

The cavity operates in the $\mathrm{TM}_{010}$ mode. This means that the maximum electric field occurs along the axis of the cavity. A potential problem results when the glass tube exits the cavity at the top and the bottom. The glass has a low dielectric constant of approximately 4 , however, the dielectric constant of the seawater sample is many times higher. Ho and Hall (1973) were concerned about this problem. Using a perturbation calculation derived by Estin and Bussey (1960) they showed that the error introduced was less than $0.1 \%$ for a cavity at $2.65 \mathrm{GHz}$. Similar calculations at $1.4 \mathrm{GHz}$ again showed the effect caused an error of less than $0.1 \%$. 


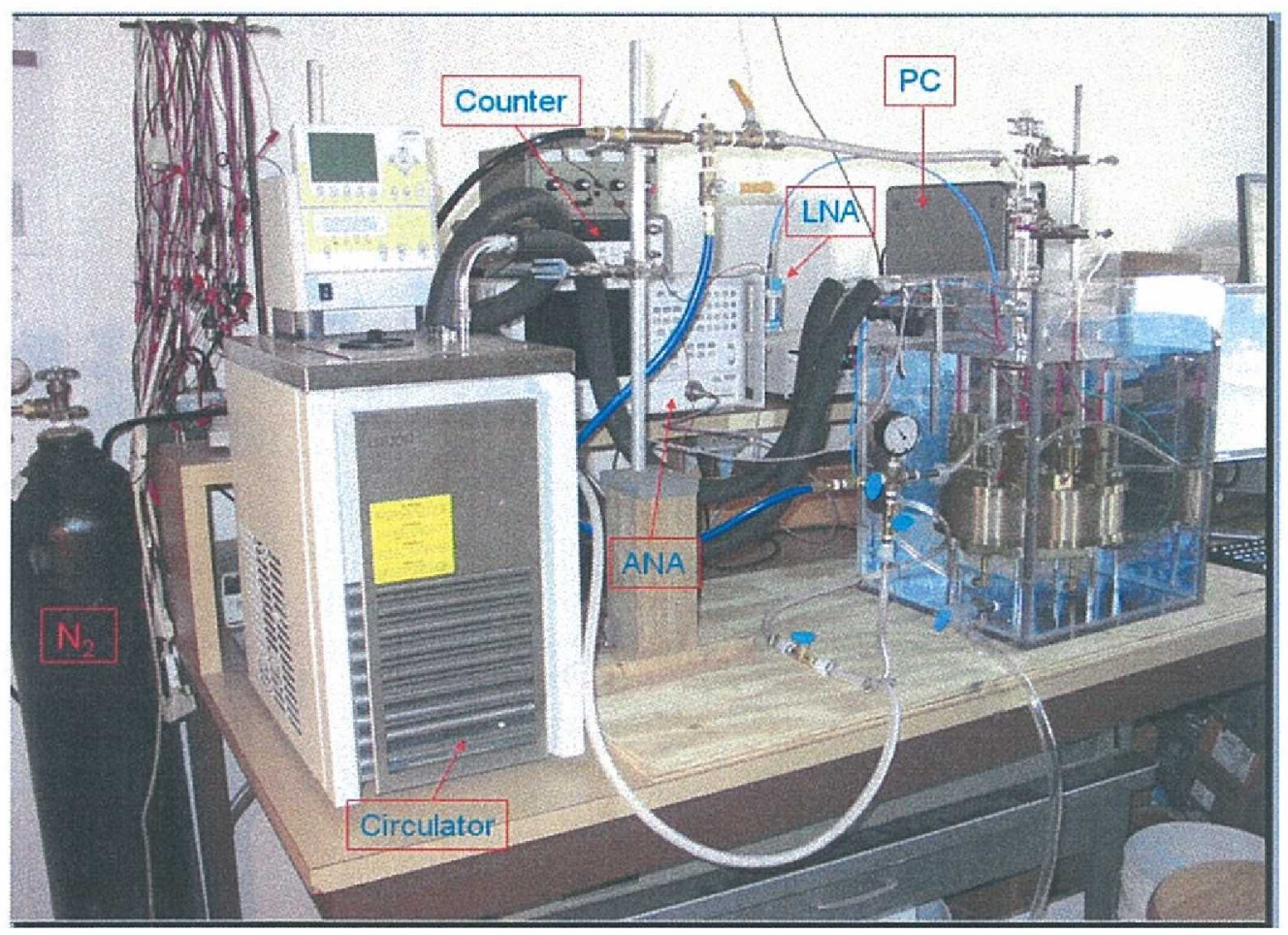

Figure 5. Experimental setup

Another consideration is the electrical conductivity between the cavity covers and the cylinder. This is a concern since the $\mathrm{TM}_{010}$ mode has currents that go up and down the walls and onto the covers. To make a proper connection, silver paste was used along the rim where the walls meet the covers. In addition, each cover was screwed down with 16 screws that were torqued to 13 in-lbs. Tests showed that increasing the pressure did not change the resonant frequency of the cavity.The cavity was kept at a stable temperature by immersing it in a bath of water and ethylene glycol. A tank was constructed of $1 / 2$ inch Lexan to hold the cavity and water/ethylene glycol

\subsection{Measurement Procedures and Data Acquisition}

The basic microwave measurement device was an HP network analyzer (Model 8722D) operated in the transmission mode (measurement of $\mathrm{S}_{21}$ ). A pair of isolators (17dB backward isolation and $0.5 \mathrm{db}$ forward attenuation), one at the input to the cavity and another at the output of the cavity were used to reduce the pulling effect of the external circuit on the cavity resonant frequency. For some measurements, $5 \mathrm{~dB}$ attenuators were used in front of the isolators to reduce the return loss of the isolators. For a few measurements, a $17 \mathrm{~dB}$ RF low noise amplifier (LNA) was used to increase the signal to noise ratio of the output signal. The amplifier was later eliminated since gain variations due to room temperature changes caused $S_{21}$ to vary. This setup is shown in Figure 7. In Appendix C.1, tables show which measurement configurations were used for different measurements. 

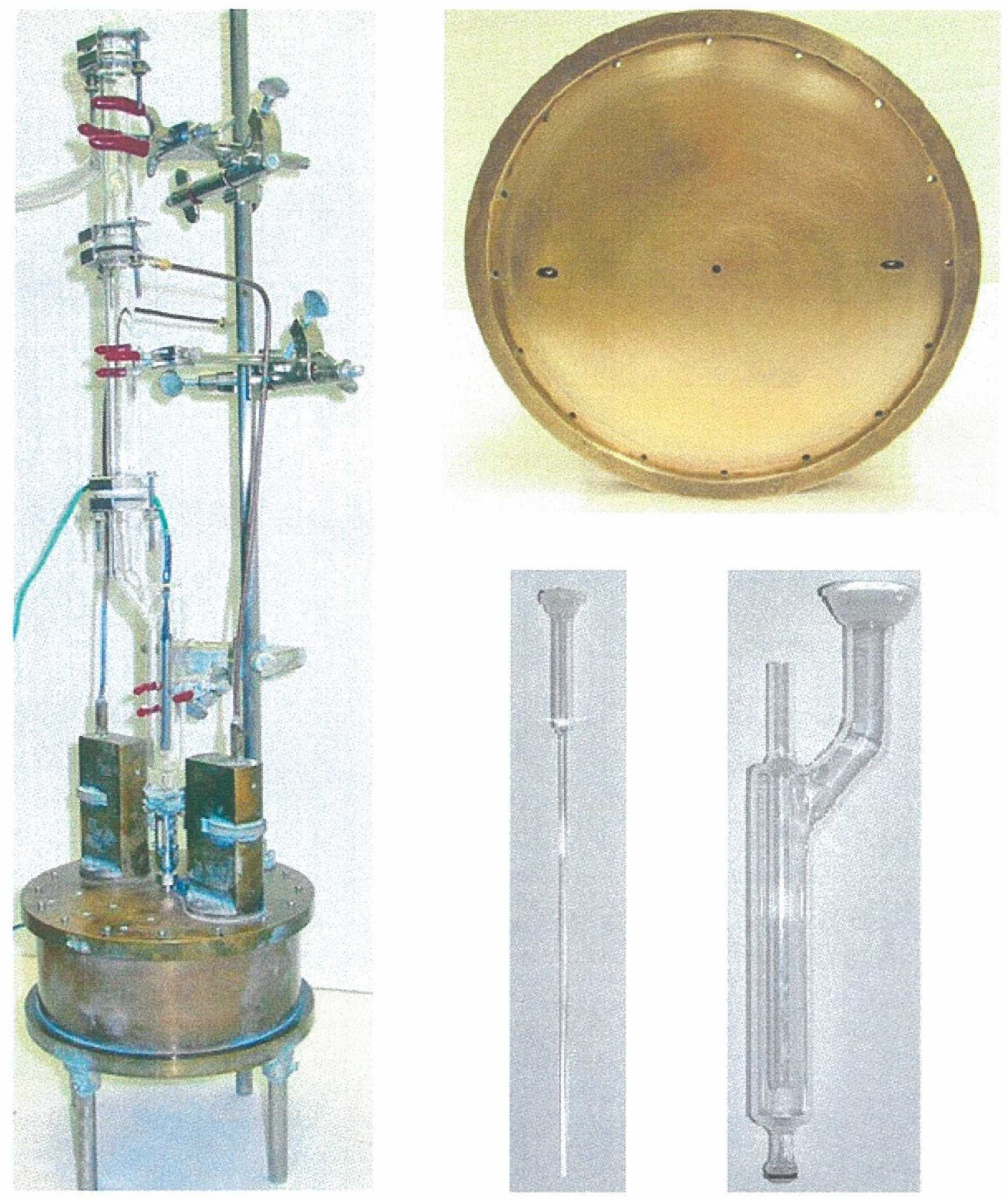

Figure 6 (a) -cavity with glass reservoir superstructure; (b) - cover of resonator showing location of coupling loops; (c) left side - thin quartz tube that holds seawater inside cavity together with quartz coupling socket; (c) right side - reservoir with interior glass tube to hold thermometer. 
The microwave power is coupled in and out of the cavity via coupling loops. These loops can be raised or lowered to decrease or increase the coupling into the cavity. Normally, a cavity in the transmission mode is operated with very weak coupling. Under these conditions, the unloaded Q of the cavity is approximately equal to the loaded Q. It is the loaded Q that is measured and the unloaded $\mathrm{Q}$ that is used in eq (2a) to find the imaginary part of the dielectric constant. This topic is discussed in more detail in Appendix D. There, it is shown that the unloaded Q does not necessarily have to be very close to the loaded $Q$ in order to be used in eq(2a). In the appendix, the $\Delta(1 / Q)$ used in eq $(2 \mathrm{a})$ is calculated for the unloaded and loaded cases. For all measurements made in this report, the two are virtually the same even though in some cases, the loaded and unloaded Q differ substantially.

The NA was set to a center frequency of $1413 \mathrm{MHz}$ with a span of $50 \mathrm{KHz}$. As measurements spanned the temperature range from $10^{\circ} \mathrm{C}$ to $35^{\circ} \mathrm{C}$, the resonant frequency varied about $700 \mathrm{KHz}$. As a result, as the temperature changed, the center frequency of the sweep had to be adjusted slightly to keep the resonant curve on the NA screen. The analyzer was set for 8 averages, 1601 points, an IF bandwidth of $100 \mathrm{~Hz}$ and a power output of $-5 \mathrm{dBm}$. This is the maximum output for this NA model. At the beginning of each measurement, a thru-calibration was done between points $\mathbf{a}$ and $\mathbf{b}$ as shown in Figure 7.

Temperature was measured using three thermistors purchased from US Sensor with an accuracy of $0.005^{\circ} \mathrm{C}$. A rectangular block of naval brass was mounted to the top and bottom of the cavity. A thermistor was tightly fitted into a cylindrical hole in each block. The third thermistor was inserted in the small glass tube inside the reservoir to measure the temperature of the seawater sample. In Figure 6(a), the thermometer is shown inserted into the reservoir tube.

Measurements were made to determine how long it would take distilled water, at room temperature, to reach the temperature of the surrounding fluid after it was introduced into the reservoir. To do this, the third thermistor was placed inside the reservoir tube with some alcohol so that the thermistor made thermal contact with the glass. Because of the length of the thermistor ( 3 inches), a large amount of distilled water had to be used to completely cover the length of the thermistor. The measurements showed that, for all temperatures in the range $\left(10^{\circ} \mathrm{C}<\mathrm{T}<35^{\circ} \mathrm{C}\right) 20$ minutes was more then enough time for the distilled water to come to equilibrium with the surrounding liquid, These measurements were taken using distilled water rather than seawater because calibrated seawater is quite expensive (approximately $\$ 150$ for a small ampoule) and the amount of seawater needed was quite large. After the initial tests with distilled water, the third thermistor was used to measure the room temperature. Inside the fixed temperature bath, repeated measurements showed that the temperature difference between the thermister on top of the cavity and the one on the bottom differed by less than $0.005^{\circ} \mathrm{C}$. Toward the end of the measurement period, the lead to the top-mounted thermister broke off flush with the thermister head making it difficult to repair. The measurements proceeded with only two thermisters: one mounted on the bottom of the cavity and one placed to measure the room temperature.

After the NA thru-calibration is completed, the Visual Basic program is started. Its measurement sequence is as follows: the NA performs 8 sweeps across 1601 frequency points. An 8 sweep average of $S_{21}$ is computed for each frequency point. By using the internal functions of the NA. 


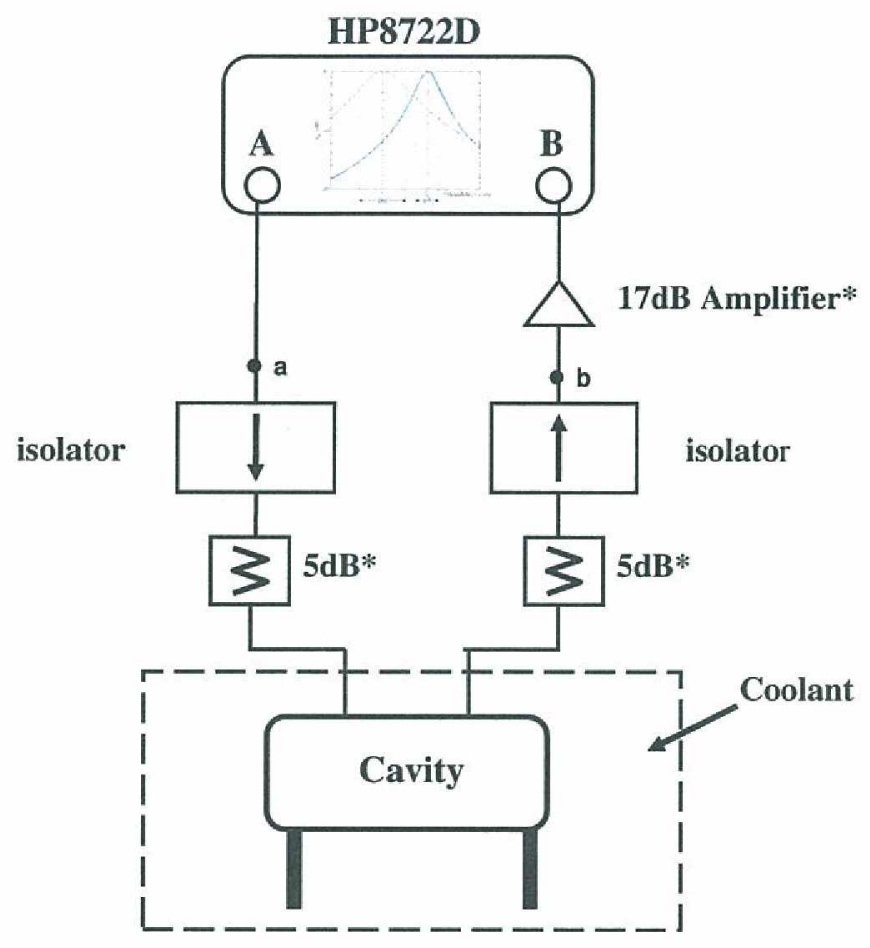

Figure 7. Measurement Setup

the center frequency, the $3 \mathrm{db}$ bandwidth and the $\mathrm{Q}$ of the resonant curve are computed. These values are only computed if the resonant curve, including the $3 \mathrm{db}$ points, are on the NA screen. Once these calculations are complete, the Visual Basic program downloads the data into a single column of an Excel spread sheet. First, the start and stop time of the measurements are downloaded, then the three temperature measurements at the start time and the three temperatures at the stop time are downloaded. This is followed by the center frequency, $3 \mathrm{~dB}$ bandwidth, the $\mathrm{Q}$ and the magnitude of $\mathrm{S}_{21}$ in $\mathrm{dB}$ at the resonance frequency. Following these quantities, the 1601 values of the magnitude of $\mathrm{S}_{21}$ in $\mathrm{dB}$ at each frequency are downloaded. These 1601 points can be used to independently determine the resonant frequency and bandwidth of the resonant curve, if desired.

The generation of one column of data represents one measurement cycle. The cycle takes a little less than 3 minutes to complete. Once it is completed, the Visual Basic program starts the next cycle. It is downloaded into the second column of the spread sheet. The process keeps on going until it is stopped.

A typical seawater experiment consists of many cycles. First, the cavity is brought to the desired temperature and allowed to stabilize. Next, 5 to 10 empty measurements, i.e., no seawater in the tube, are made. The seawater is then put into the glassware by opening the glass cap at the top of the glass structure (see Figure 6(a)) and inserting a small nylon tube down to the top of the 
cavity. The nylon tube is used so that the walls of the glassware won't be contaminated with seawater. Only the tube and the very bottom of the glassware need to be washed after the experiment is finished. The seawater is inserted into the tube by using a syringe with a $50 \mu \mathrm{m}$ filter on it. The glass cap is replaced, and the seawater is allowed to stand about 20 minutes until it reaches the temperature of the surrounding liquid. Because of the viscous nature of seawater, it usually does not enter the tube. To start the measurements, $\mathrm{N}_{2}$ ( at a pressure of about 2 atmospheres) is used to push the seawater into the sample tube. The pressure is kept constant until all the seawater leaves the reservoir and the tube is empty. Next the pressure is released and distilled water is added to clean the cavity. Once the distilled water is added, the pressure is turned back on. Enough distilled water is added so as to clean the glass surface that has been in contact with the seawater. This cleaning procedure can take from 15 to 30 minutes depending on how much distilled water is added. The last step is a set of empty tube measurements to confirm that the cavity has indeed returned to its empty state. The whole procedure to measure the seawater at one temperature and one salinity can take from 1 to 3 hours. It should be pointed out that, after the tube is cleaned with distilled water, the resonant frequency does not usually return to exactly the same value it had when the experiment was started a few hours earlier. The resonant frequency of the cavity exhibits a slow drift over time. This drift is discussed in Appendix C.3.

An Excel worksheet has been coded to plot measured variables as a function of time. Some data from an experiment performed on May 19, 2008 to measure the dielectric constant of seawater having a salinity of $34.999 \mathrm{psu}$ and temperature of $25^{\circ} \mathrm{C}$ is shown in Figures 8 and 9. In Figure 8 , the resonant frequency and the $\mathrm{Q}$ are shown as a function of time. Each diamond on the plot represents the value of the plotted variable in a particular column of the spread sheet. The time scale is expressed in terms of the number of measurement cycles. Thus if each experiment takes approximately 3 minutes, the value 10 on the time scale represents 30 minutes. The total time shown for the experiment represents about $3 \frac{1}{2}$ hours $(3 \times 70=210$ minutes $)$. In Figure 9 , the cavity temperature and the room temperature are shown. We see that over the total time required for the measurements $\left(3 \frac{1}{2}\right.$ hours), the cavity temperature remained stable at $25.04 \pm 0.015^{\circ} \mathrm{C}$ while the room temperature varied. The wave like character of the cavity temperature graph reflects the cyclic switching of the circulator between cooling and heating.

Consider the measurements of Figure 8 again. At the beginning, the sample tube in the cavity is empty. An atmosphere or two of $\mathrm{N}_{2}$ is applied to ensure that the sample tube is empty at the start of the experiment. As a result, the Q remains about 7800 for the first 18 cycles. At approximately the $10^{\text {th }}$ or $11^{\text {th }}$ cycle, the seawater is added to the reservoir, however it does not enter the tube immediately since it is too viscous. After waiting 7 to 8 cycles for the seawater to reach $25^{\circ} \mathrm{C}$ (about 20 minutes), a pressure of 2 atmospheres of $\mathrm{N}_{2}$ is applied. It pushes the seawater into the tube and the $\mathrm{Q}$ falls to about 4600. Now 6 measurement cycles occur before all the seawater is pushed through the tube. At this time, the pressure is released and distilled water is added. The $\mathrm{Q}$ levels out at slightly less than the free space $\mathrm{Q}$ since distilled water has a very low loss. After about 12 cycles the distilled water is completely expelled from the reservoir and the tube. The $\mathrm{Q}$ returns to the empty state. If it does not, a second washing with distilled water is made. 

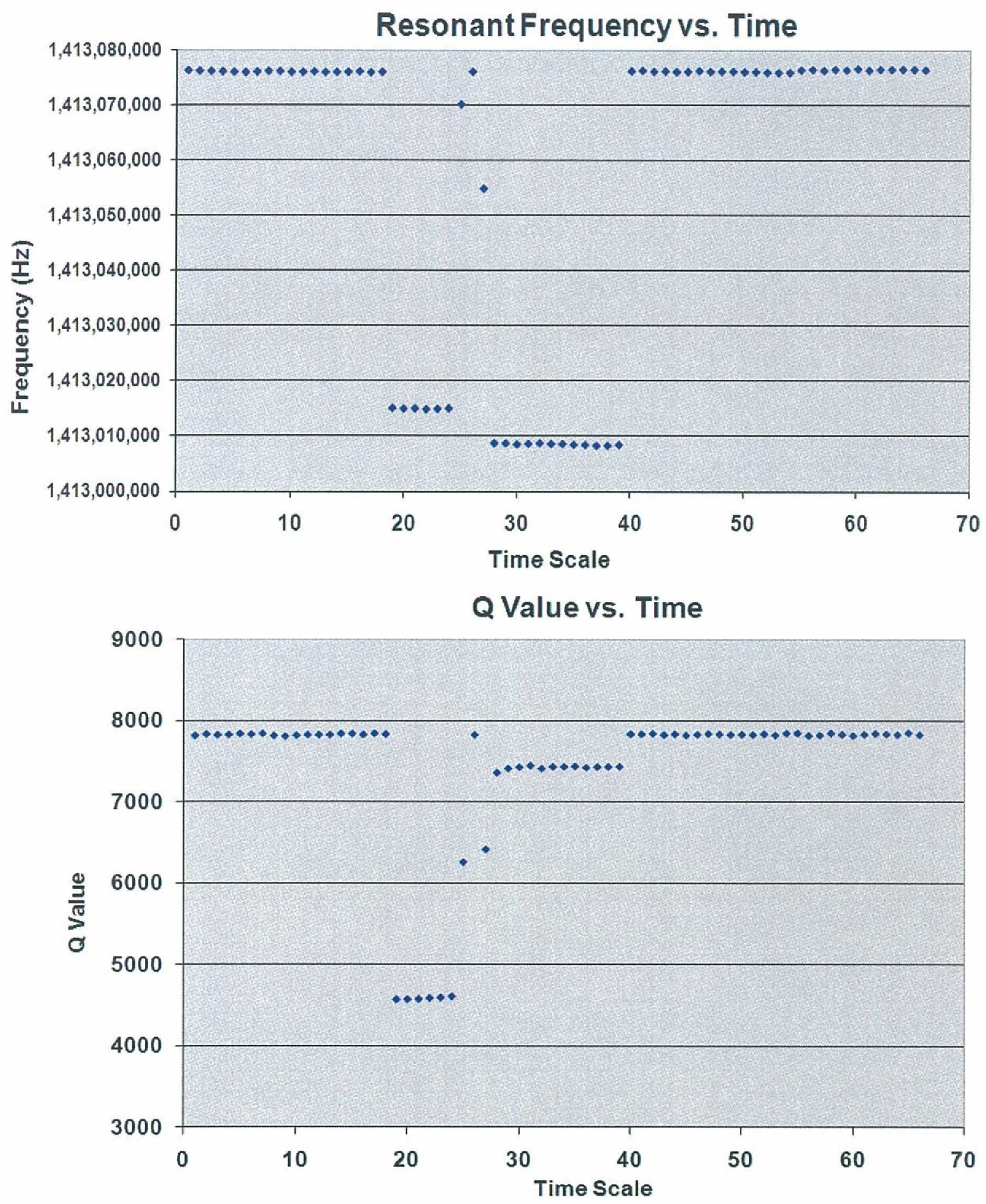

Figure 8. Measurements of the resonant frequency and $\mathrm{Q}$ as a function of time. 

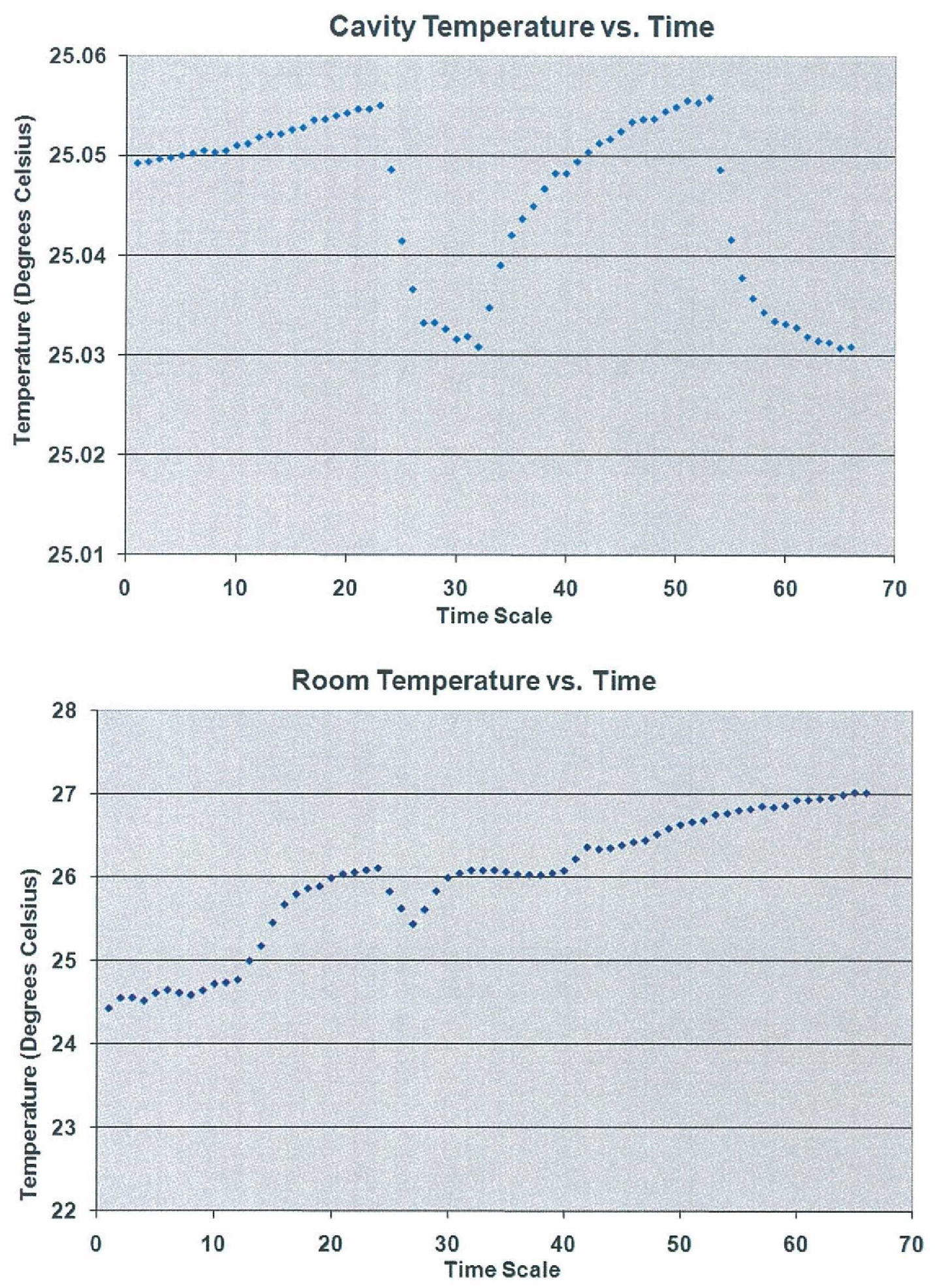

Figure 9. Measurements of cavity and room temperature as a function of time. 
To compute the real part of the dielectric constant of seawater, we will need the change in the resonant frequency when the tube is empty and when it has seawater in it. The change in $1 / \mathrm{Q}$ will be needed to compute the imaginary part of the dielectric constant. Three points are selected from the empty measurements right before the transition to the seawater state. These are recorded as sample 1, 2 and 3 in Table 1 under Empty Measurements. Three points (measurement cycles) are selected from the 6 cycles when the seawater is in the tube and entered into Table 1 under Seawater Measurements. The cycles closest to the transition point are usually chosen. Finally three points from the empty portion - after the distilled water has been expelled from the tube - are chosen and entered into the table under Ending Empty Measurements. In all three cases, the three points chosen are contiguous points. The average of the three samples is computed in each case. These averages are the values that will be used in the calculation. The standard deviation is also found. This provides an important check. If it is too large, other points will be chosen and if this is not satisfactory the measurement will be discarded.

\section{EXPERIMENTAL DATA}

\section{EMPTY MEASUREMENTS}

$\begin{array}{lccccc}\text { May 19,08 } & \text { Res. Freq }(\mathbf{H z}) & \mathbf{Q} & \text { Cavity T } & \text { Room T } & \text { Time } \\ \text { Sample 1 } & 1,413,076,041 & 7,827 & 25.05 & 25.68 & 11: 47: 10 \\ \text { Sample 2 } & 1,413,075,866 & 7,843 & 25.05 & 25.80 & 11: 49: 59 \\ \text { Sample 3 } & 1,413,075,964 & 7,832 & 25.06 & 25.88 & 11: 52: 47 \\ \text { Average } & 1,413,075,957 & 7,834 & 25.06 & 25.79 & 11: 49: 59 \\ \text { Stdev } & 88 & 8 & 0.00 & 0.10 & \end{array}$

SEAWATER MEASUREMENTS (34.997psu)

$\begin{array}{lccccc}\text { May 19,08 } & \text { Res. Freq } \mathbf{~ ( H z ) ~} & \mathbf{Q} & \text { Cavity T } & \text { Room T } & \text { Time } \\ \text { Sample 1 } & 1,413,015,075 & 4,581 & 25.05 & 26.03 & 12: 01: 14 \\ \text { Sample 2 } & 1,413,014,899 & 4,593 & 25.05 & 26.06 & 12: 04: 03 \\ \text { Sample 3 } & 1,413,014,980 & 4,599 & 25.06 & 26.09 & 12: 06: 51 \\ \text { Average } & 1,413,014,985 & 4,591 & 25.05 & 26.06 & 12: 04: 03 \\ \text { Stdev } & 88 & 9 & 0.00 & 0.03 & \end{array}$

\begin{tabular}{lcccccc}
\multicolumn{6}{c}{ ENDING EMPTY MEASUREMENTS ( Once Distilled Water Cleaning) } \\
May 19,08 & Res. Freq (Hz) & Q & Cavity T & Room T & Time \\
Sample 1 & $1,413,076,114$ & 7,833 & 25.05 & 26.09 & $12: 54: 38$ \\
Sample 2 & $1,413,076,208$ & 7,834 & 25.05 & 26.22 & $12: 57: 27$ \\
Sample 3 & $1,413,076,020$ & 7,840 & 25.05 & 26.36 & $13: 00: 16$ \\
Average & $1,413,076,114$ & 7,835 & 25.05 & 26.23 & $12: 57: 27$ \\
Stdev & 93 & 4 & 0.00 & 0.14 &
\end{tabular}

Table 1. Experiment data from a measurement made on May 19, 2008 of seawater having salinity of 34.999 psu at a temperature of $25^{\circ} \mathrm{C}$. 


\subsection{Validity of Perturbation Theory}

The perturbation equations presented in eqs(2a) and (2b) have existed in the literature since resonant cavity measurements were first used to measure dielectric constants more than fifty years ago. In Chen et al (2004), the usual derivation of the formulas is given. This derivation has several deficiencies. First, it assumes that the empty cavity loss can be neglected, and second, it doesn't indicate how to compute an error term. In appendix B, a derivation of the perturbation equations is presented that accounts for the empty cavity losses. It also gives first order error terms that can be computed.

The perturbation equations derived in Appendix B are identical to those given in eqs.(2a) and (2b). They are:

$$
\varepsilon^{\prime}-1=2 C \frac{\Delta f}{f} \quad \varepsilon^{\prime \prime}=C \Delta(1 / Q)
$$

The only difference between the above equations and those given in eqs(2a) and (2b) is the calibration constant $C$. In Appendix B, $C$ is given as a product

$$
\mathrm{C}=\chi C_{0}
$$

where $C_{0}$ is a constant of the system while $\chi$ depends on the sample properties and the temperature. The constant $C_{0}$ is the ratio of the average energy in the empty cavity to the average energy in the empty tube. It is a constant of the system; it only changes slightly as the temperature changes and the cavity expands or contracts a slight amount. The multiplicative factor $\chi$, on the other hand, is an energy storage factor which changes with the type of liquid being measured and the temperature. If the perturbation is small enough then $\chi$ is close to 1 and the calibration coefficient is close to $C_{0}$, a constant of the system. In Appendix B, an expression for $\chi$ is given in terms of the fields in the cavity when it is empty and when it has a liquid in it. By solving the boundary value problem for the fields in the cavity under both these conditions, the energy factor $\chi$ can be computed. It is seen that as the inner tube radius becomes smaller (smaller sample size) the factor $\chi$ goes to 1 .

\subsection{Calibration}

Before seawater dielectric measurements can be made, the calibration coefficient, $C$, must be known. From eq(3), it is seen that $C$ depends on the dielectric constant of the sample itself unless $\chi \approx 1$. Therefore, to make the formulas useable, it must be required that $\chi \approx 1$. As has been pointed out previously, $\chi$ can be made close to 1 by making the tube radius small enough. More discussion on this point occurs later in this section.

As was mentioned earlier in the report, the value of $C$ can be determined by two calibration methods: absolute and relative calibration. For both methods the assumption is made that $C \approx$ $C_{0}$. For the absolute calibration method $C_{0}$ is calculated from its definition that is given in Appendix B. This requires detailed electromagnetic modeling of the cavity and accurate knowledge of the sample tube ID. The relative approach uses another reference liquid whose dielectric constant is accurately known a priori. The method is quite accurate since it is less 
dependent on any imperfections in the cavity and it does not require accurate knowledge of the sample tube ID. This method, however, depends on an accurate knowledge of the reference liquid's dielectric constant. Since accurate measurements of methanol's dielectric constant for the temperature range and the approximate frequency of interest are available, the relative approach has been used in these measurements.

\subsubsection{Calibration Liquids}

To use the relative calibration technique, the complex dielectric constant of some liquid must be accurately known. Consequently, the total accuracy of our measurements will depend on the accuracy of the seawater measurements, as well as, on the accuracy of the calibration coefficient. The accuracy of the calibration coefficient, in turn, depends on the accurate knowledge of the dielectric constant of the reference liquid. As will be demonstrated, it is also necessary to have a liquid whose dielectric constant is not too different from the dielectric constant of seawater.

Two easily obtainable liquids that meet these criteria are methanol and distilled water. Gregory and Clark (2009) have made accurate measurements of methanol at 20 individual frequencies from 0.1 to $5.0 \mathrm{GHz}$ for a temperature range from $10^{\circ} \mathrm{C}$ to $50^{\circ} \mathrm{C}$ in increments of 5 degrees. Their measurements have an accuracy of approximately $0.3 \%$ in most cases of interest to us. The measurements have been made with coaxial transmission lines of different lengths and a coaxial reflection device. In all cases each result has been checked using two measurement devices. The other liquid is distilled water. Kaatze (1989) has made measurements of distilled water over a frequency range from $1.1 \mathrm{GHz}$ to $57 \mathrm{GHz}$ for various temperatures in our range by using a microwave double beam interferometer. From these values he has constructed a model function. He claims an accuracy of better than $1 \%$ for the real part and $3 \%$ for the imaginary part in the frequency range from $1.1 \mathrm{GHz}$ to $5.3 \mathrm{GHz}$. Since the accuracy of the methanol measurements is better than the distilled water measurements, we will use methanol as our calibration liquid. Distilled water, however, does have an advantage since its dielectric constant is closer to the dielectric constant of seawater.

\subsubsection{Variation in Calibration Coefficient with Liquid Type}

In this subsection, we develop the method for the use of a reference liquid to calibrate the sample equations and the dependence of this procedure on the choice of the calibration liquid. The calibration coefficient, $C$, appearing in eq(4) is a product of $C_{0}$ and $\chi$, a factor that depends on the dielectric constant of the liquid being measured. In relating the calibration measurements to the seawater measurements, there are two systems of equations to consider: the sample equations and the reference equations. The sample equations are:

$$
\varepsilon_{s}^{\prime}-1=2 C_{s} \frac{\Delta f_{s}}{f_{s}} \quad \varepsilon_{s}^{\prime \prime}=C_{s} \Delta\left(1 / Q_{s}\right)
$$

and the equations for the reference liquid are:

$$
\varepsilon_{r}^{\prime}-1=2 C_{r} \frac{\Delta f_{r}}{f_{r}} \quad \varepsilon_{r}^{\prime \prime}=C_{r} \Delta\left(1 / Q_{r}\right) .
$$




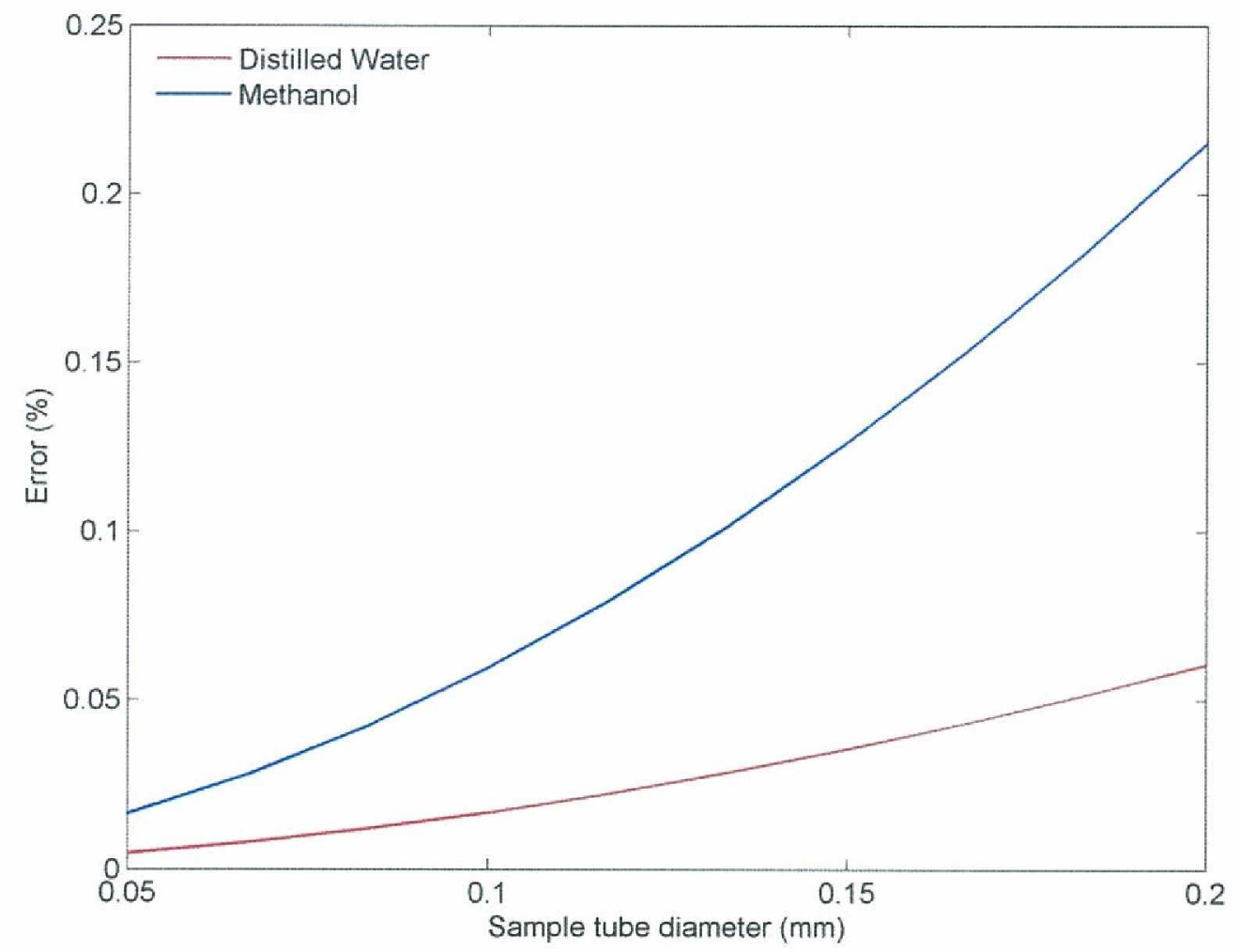

Figure 10. Plot of Error $(\%)=100 \times(\chi-1)$ versus the inner diameter of the sample tube.

By employing eq(4), the calibration constants are each related to $C_{0}$ as follows:

$$
C_{s}=\chi_{s} C_{0} \quad C_{r}=\chi_{r} C_{0}
$$

Combining these the two equations above gives

$$
C_{s}=\left(\chi_{s} / \chi_{r}\right) C_{r}
$$

To determine the calibration coefficient, $C_{s}$, for use in the sample measurement equations, the following procedure is followed: first, use either the known values of $\varepsilon_{r}^{\prime}$ or $\varepsilon_{r}^{\prime \prime}$ in eq(6) to determine $C_{r}$ and second, assuming $\chi_{s} / \chi_{r}$ is known, compute $C_{s}$ from eq(8). The ratio of the $\chi$ 's is not known but it can be made close to 1 . In practice, this is done by making the inner tube diameter small enough and choosing a reference liquid with a dielectric constant close to that of the sample. An approximate expression for $\chi$ is given in Appendix B. Plots of this ratio as a function of tube radius with methanol as the reference liquid at $20^{\circ} \mathrm{C}\left(\varepsilon_{r}=27.96+i 11.20\right)$ and with distilled water as a reference liquid at $20^{\circ} \mathrm{C}\left(\varepsilon_{r}=79.75+i 5.83\right)$ are shown in Figure 10. The sample dielectric constant has been assigned a large value $\left(\varepsilon_{s}=100+i 100\right)$ to assume the worst possible case. For real seawater samples, the corresponding error will be slightly smaller. 
An examination of Figure 10 shows that for a tube with an inner diameter of $0.1 \mathrm{~mm}$, the error in approximating $C_{s}$ by $C_{r}$ for methanol is about $0.06 \%$ which is negligible. From this point on, we will refer to a single calibration coefficient $C$, determined from measurements of methanol as reference liquid.

It should be pointed out that if $\chi_{s} / \chi_{r}$ is not chosen close to 1 , then the measurement procedure will be a function of the dielectric constant being measured making the value of $\chi_{s} / \chi_{r}$ different for each value of $\varepsilon_{s}$ - not a good situation. Note from Figure 10 that when distilled water is used, a larger tube radius can be employed for same error. Normally distilled water would have been chosen for a calibration liquid but values of its dielectric constant at the frequency and temperatures of interest are not known with the same accuracy as for methanol.

\subsubsection{Calibration Measurements Using Methanol}

Multiple measurements of methanol were made over the period from January 26, 2008 to May 13, 2008 to determine a calibration coefficient for tube \#12. Table C.1-1 in Appendix C.1 shows the microwave network (see Figure 7) that was used for each measurement. The table indicates that isolators were always employed. For the data taken in April and May of 2008, 5 dB attenuators were also used. These microwave devices were used to isolate the cavity from its external environment. The strategy was not completely successful in stabilizing the long term frequency drift, possibly due to the fact that the temperatures of the attenuators and the isolators also changed as the room temperature varied.

The measurements of resonant frequency and $\mathrm{Q}$ for the empty cavity and for the cavity with methanol in it are shown in Table 2. The methanol used was purchased in early January, 2008 from Fisher Scientific. Its product number was A935-4 and it was rated as having low water content. In Table 2, the difference frequency, $\Delta f_{r}$, and the difference $\Delta\left(1 / Q_{r}\right)$ are computed for use in eq(6). The calibration coefficient, $C$ can be calculated using either the equation determining $\varepsilon_{r}^{\prime}$ or the equation determining $\varepsilon_{r}^{\prime \prime}$ in eq(6). We will use both equations and obtain two values of $C$. They are referred to in the Table 2 as C1 (determined from the $\varepsilon_{r}^{\prime}$ equation) and $\mathrm{C} 2$ (determined from the $\varepsilon_{r}^{\prime \prime}$ equation). The closeness of $\mathrm{C} 1$ and $\mathrm{C} 2$ will be used as a measure of the self consistency of the measurement.

Before calculating $\mathrm{C} 1$ and $\mathrm{C} 2, \varepsilon_{r}^{\prime}$ and $\varepsilon_{r}^{\prime \prime}$ must be determined from the tables of Gregory and Clarke (2009). Since the tables do not provide the dielectric constant for a frequency of 1.413 $\mathrm{GHz}$, interpolation will be used. This process is performed by sixth degree polynomial interpolation, and the results are given in the caption to Figure 11. Now that the complex dielectric constant of methanol at $20^{\circ} \mathrm{C}$ has been determined, the formulas in eq(6) can be used to determine $\mathrm{C} 1$ and $\mathrm{C} 2$. The results of all the methanol measurements that have been made are listed in Table 2. From these experimental results, the values of $\mathrm{C} 1$ and $\mathrm{C} 2$ are also computed and listed in the table.

Rather than use all the data in Table 2 to determine $C$, the percent difference between $\mathrm{C} 1$ and $\mathrm{C} 2$ will be used to eliminate some data points as being too inconsistent. The quantity $100 \times(\mathrm{C} 1 / \mathrm{C} 2-1)$ 


\begin{tabular}{|c|c|c|c|c|c|c|c|c|c|}
\hline Date & Empty $f_{0}(\mathrm{~Hz})$ & $\begin{array}{c}\text { Methanol } \\
\mathrm{f}_{\mathrm{r}}(\mathrm{Hz})\end{array}$ & $\mathrm{f}_{\mathrm{r}}(\mathrm{Hz})$ & \begin{tabular}{|c|} 
Empty \\
$\mathrm{Q}_{0}$ \\
\end{tabular} & \begin{tabular}{|c|} 
Methanol \\
$\mathbf{Q}_{\mathrm{r}}$ \\
\end{tabular} & $\Delta\left(1 / Q_{r}\right)$ & C1 & 2 & \begin{tabular}{|c|}
$|\mathrm{C} 1 / \mathrm{C} 2-1|$ \\
$\times 100$ \\
\end{tabular} \\
\hline $01 / 26 / 08$ & $113,279,303$ & $1,413,255,197$ & 4,107 & 7,741 & 6,979 & $1.4094 \mathrm{E}-05$ & 790,280 & 794,664 & $0.552 \%$ \\
\hline$/ 08$ & 78,552 & $1,413,254,151$ & & 7,752 & 81 & $1.4241 \mathrm{E}-05$ & 780 & & $729 \%$ \\
\hline $01 / 26 / 08$ & $413,278,164$ & $413,254,037$ & 127 & 7,766 & & 1.421 & 7 & & $.243 \%$ \\
\hline & & 56 & & 79 & & 05 & & & $03 \%$ \\
\hline & & 35 & & & & & & |792,553 & $88 \%$ \\
\hline & 1,4 & 1,4 & & & & -05 & 793,846 & 828,648 & $.200 \%$ \\
\hline & 77 & 1,41 & & 7,675 & & -05 & 781,850 & 789,864 & $1.015 \%$ \\
\hline & 42 & 1,41 & & 7,658 & & $=05$ & 742 & 796,473 & $6.774 \%$ \\
\hline $02 /$ & 615 & 1,41 & & 7,632 & 6,899 & -05 & 786 & 804,696 & $2.244 \%$ \\
\hline $04 / 10 / 08$ & 48 & 1,41 & & 7,375 & 6,673 & E-05 & 787,588 & 785 & $0.281 \%$ \\
\hline $04 / 19 / 08$ & 681 & 1,41 & 23,202 & 7,883 & 7,103 & E-05 & 821 & 628 & $2.168 \%$ \\
\hline $04 / 24 / 08$ & 2,694 & 1,41 & 23 & 7,870 & 86 & $=-05$ & 812 & 796,529 & $1.983 \%$ \\
\hline $04 / 24 / 08$ &, 573 &, 256 & 23,317 & 7,865 & 7,080 & $\mathrm{E}-05$ & 816,994 & 794,529 & $2.827 \%$ \\
\hline $04 / 25 / 08$ & 693 & 1,086 & 23,607 & 7,869 & 7,080 & 1.41 & 806 , & 790 & $2.099 \%$ \\
\hline $04 / 29 / 08$ & 919 & 506 & 24, & 7,633 & 91 & E-05 & 780 & & $1.706 \%$ \\
\hline & & 1,4 & 24 & 7,569 & 8 & E-05 & 787 & 793 & $0.714 \%$ \\
\hline & & 1,4 & & 7,573 & 3 & 1.42 & 785 & 783,255 & 0.2 \\
\hline & & & 23 & 7,846 & 8 & 1.40 & 797 & 797 & 0.0 \\
\hline &, 702 & 333 & & 7,852 & 7,074 & 1.40 & 798 & 799 & $0.218 \%$ \\
\hline verage & $1,413,237,763$ & $1,413,213,672$ & 24,091 & 7,719 & 6,962 & E-05 & & 795,088 & \\
\hline
\end{tabular}

Table 2. Chart of measured methanol data and calculated values of $\mathrm{C} 1$ and $\mathrm{C} 2$ for each data point. All measured data with $100 \times(\mathrm{C} 1 / \mathrm{C} 2-1)<1.5 \%$ are marked in yellow.

\begin{tabular}{|c|c|}
\hline \multicolumn{2}{|c|}{ Calibration Analysis, C1 } \\
\hline Average & 788,249 \\
\hline Stdev & 5996 \\
\hline Error (\%) & $0.76 \%$ \\
\hline
\end{tabular}

\begin{tabular}{|c|c|}
\hline \multicolumn{2}{|c|}{ Calibration Analysis, C2 } \\
\hline Average & 791208 \\
\hline Stdev & 5333 \\
\hline Error (\%) & $0.67 \%$ \\
\hline
\end{tabular}

Table 3. The charts above give the average and standard deviation of $\mathrm{C} 1$ (Table 3a) and of C2 (Table $3 \mathrm{~b}$ ) over measurements that have a consistency of better than $1.5 \%$. The error $(\%)$ $=($ Stdev/Average $) \times 100$.

is computed in the last column. Only those experiments having $C$ 's that differ by less than $1.5 \%$ will be used in the calculation of the average $C$ and its standard deviation. These experiments are shown in yellow in Table 2. The average values of $\mathrm{C} 1$ and $\mathrm{C} 2$ over measurement values that have a consistency of at least $1.5 \%$ are given in Table 3 . Also shown in the tables is the standard deviation over the consistent measurement points. The value of $\langle C l\rangle$ will be used as $C$ i.e., $C=$ $<C l>$.

\subsection{Seawater Dielectric Measurement Data}

In this section, the measurement of seawater samples will be discussed. The section first addresses where the seawater samples were obtained. Following this, the measurement configuration and times the measurements were taken are discussed. Next, the actual data with 


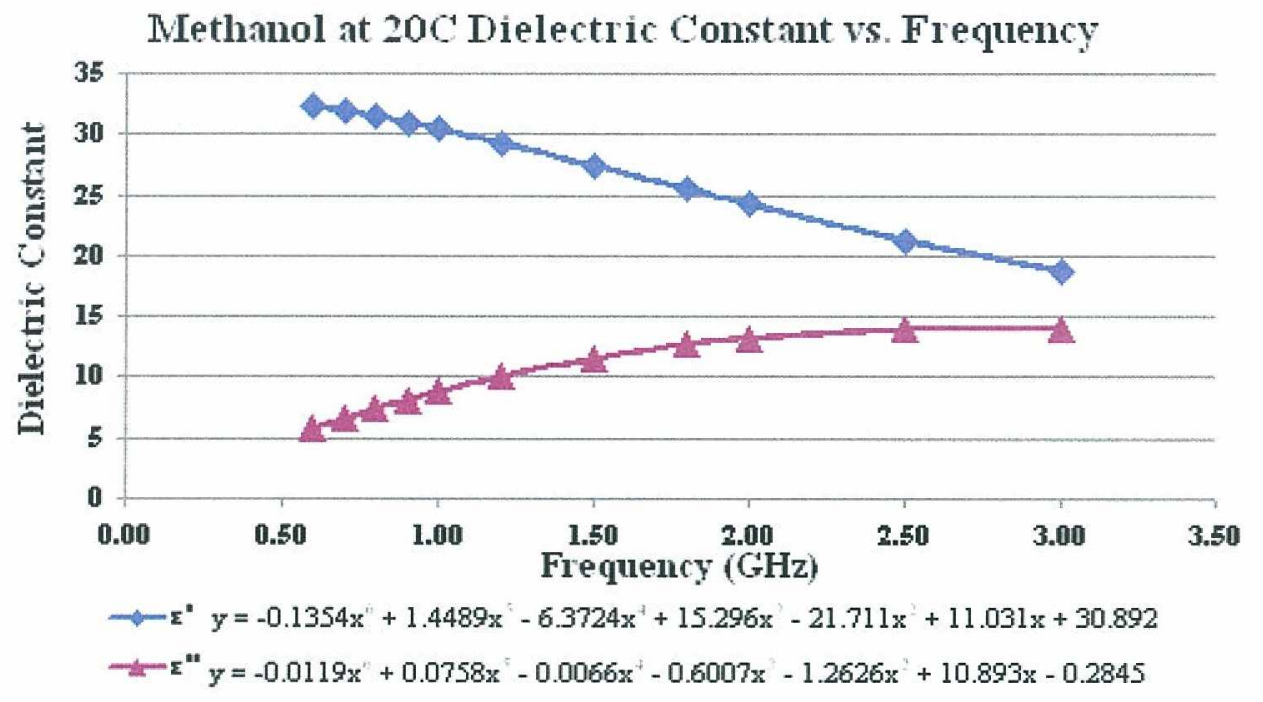

Figure 11. Polynomial fits to the Gregory and Clarke data for methanol at $20^{\circ} \mathrm{C}$. At a frequency of $1.413 \mathrm{GHz}$ the interpolated values are: $\varepsilon_{r}^{\prime}=27.96$ and $\varepsilon_{r}^{\prime \prime}=11.20$.

its means and variances are presented. This is followed by an analysis of the errors.

\subsubsection{Seawater Samples}

The seawater was purchased from Ocean Scientific International Limited (OSIL) located in Havart, Hampshire in the United Kingdom. They sell IAPSO Standard Seawater. The standard seawater as approved by the International Association for Physical Sciences of the Ocean (IAPSO) is the only transfer standard for Practical Salinity Scale that is recognized by the major oceanographic bodies.

The samples are prepared from surface seawater collected from the North Atlantic and then pumped through filters $(0.2 \mathrm{~mm})$ into a PVC lined tank and thoroughly mixed. Once purified by filtration and UV radiation, distilled water is added to adjust the salinity and the water is sealed in pre-washed glass bottles (capacity $200 \mathrm{~cm}^{3}$ ). The ratio of electrical conductivity of each batch of seawater to the conductivity of a defined standard potassium chloride $(\mathrm{KCl})$ solution is used to measure the salinity in psu. This is done by using a high precision salinometer.

The seawater is sold in several standard packages. GW purchased the linearity pack. It consists of 10 bottles ( $200 \mathrm{ml}$ each) of seawater having the following salinities: 4 bottles of $35 \mathrm{psu}, 2$ bottles of $38 \mathrm{psu}, 2$ bottles of $30 \mathrm{psu}$ and 2 bottles of $10 \mathrm{psu}$. Two seawater linearity packs have been purchased: one in November of 2002 and another in March, 2008. OSIL says that the seawater has negligible change in salinity in a shelf life of 3 years. Bacon et al (2007) have shown that the salinity changes by less than .001 in 282 weeks. Only seawater purchased in November, 2002 has been used in the experiments reported here. 


\subsubsection{Seawater Measurements}

The seawater dielectric constant measurements reported here have been made over the period from December, 2007 to June, 2008. Seawater having salinities of 30.002, 34.997 and 38.274 psu was measured. The seawater having salinity of 30.002 psu was measured during the period from December, 2007 to February, 2008; the 34.997 psu seawater was measured from May to June 2008 and the seawater of salinity 38.274 psu was measured from March to May, 2008.

During this time, only one quartz tube (\#12) was employed and thus the methanol calibration measurements can be applied to all measurements. As noted previously, calibration measurements were made from January, 2008 to May, 2008. As more calibration data became available, the calibration coefficient was recomputed. As a result, measurement data appearing in Lang et al (2008a, 2008b) may differ slightly from the results given in this report. In addition, other seawater measurements have also been added to the data set since the publication of those papers. Although the curves look slightly different than the ones appearing in the conference papers, the conclusions published are essentially the same.

The configuration of the microwave network connecting the NA to the cavity has been changed during the experiments. The network configuration for each seawater measurement made is shown in Appendix C. 1 in Tables C.1-2 for seawater of salinity of 30.002 psu, in Table C.1-3 for seawater of salinity of 34.997 and in Table C.1-4 for seawater of salinity of 38.274 psu.

The measurements of 30 psu seawater started in December of 2007. At that time, two isolators were employed at the input and output of the cavity. Previous experimentation had shown that the long term stability of the resonant frequency measurements was less influenced by external temperature changes when isolators were used. A $17 \mathrm{~dB}$ amplifier was also employed to increase the signal-to-noise ratio. After the measurements were made in February, 2008 it was decided to eliminate the amplifier. Since the amplifier was in the calibration loop, changes in amplifier gain caused by room temperature changes, affected the NA calibration. The change in calibration did not affect the cavity resonant frequency or the Q. The resulting measurements made while the amplifier had been in the circuit were accurate.

Measurements made during March and April of 2008 employed only the isolators. Before the May measurements began attenuators were added in front of the isolators to reduce their return loss. The 34.997 psu measurements employed isolators and attenuators since they were made in May and June, 2008. The 38.274 psu measurements were made in March and in May, 2008. The March measurements used only isolators while the May measurements used isolators and attenuators.

The individual seawater measurements are given in Appendix C.2 in 3 tables labeled Table C.2-1 for the 30.002 psu data, Table C.2-2 for the 34.997 psu data and Table C.2-3 for the 38.274 data. In these tables the date for each measurement is given along with the change in resonant frequency, $\Delta f$, and the change in $1 / Q, \Delta(1 / Q)$. Using these quantities together with the calibration constant $C(C=788,249)$ in eq(2a) and eq(2b), the real and imaginary parts of the complex dielectric constant of seawater are calculated. They are compared with the values predicted by the Klein and Swift (KS) model function for seawater in the last two columns. It should be pointed out that in making the calculation of the imaginary part, the measured Q is 
used. It is actually the unloaded Q that should have been used. In Appendix D, it is shown for a representative subset of measurements that there is virtually no difference between $\Delta(1 / Q)$ evaluated with the loaded Q or the unloaded Q. Although the loaded and unloaded Q values differ from each other, the difference cancels out when computing $\Delta(1 / Q)$.

An examination of the seawater measurement tables in Appendix C.2 shows that the change in frequency, $\Delta f$, lies between 58 to $67 \mathrm{KHz}$. These frequency differences must be accurate to three places to give real dielectric values that have errors of less than $0.2 \%$. The data also shows that the empty Q is approximately 7800 while the Q's with the seawater vary from 4000 to 5000 depending on the salinity of the seawater. Similarly, the Q must be accurate to three places to give the required accuracy. The means and standard deviations for each measurement set at a given salinity and temperature are presented in Table 4 . The mean values at a given salinity are then used to construct graphs of real and imaginary parts of the dielectric constant as a function of temperature for a given salinity. The standard deviation of each measurement point is shown by a vertical bar that goes up by $\sigma$ and down by $\sigma$ where $\sigma$ is the standard deviation. The KS model function has been drawn on the same plots for comparison purposes. These plots are shown in Figure 12 for salinity of 30.002 psu, in Figure 13 for salinity of 34.997 psu, and in Figure 14 for salinity of 38.274 psu. It should be pointed out that an average of 3.3 points per measurement has been used to compute these statistics.

\begin{tabular}{|c|c|c|c|c|c|}
\hline \multicolumn{7}{|c|}{ GWU Seawater Data } \\
\hline Temp (Celsius) & psu & $\varepsilon^{\prime}$ (Mean) & $\varepsilon^{\prime}$ (Standard Deviation) & $\varepsilon^{\prime \prime}$ (Mean) & $\varepsilon^{\prime \prime}$ (Standard Deviation) \\
\hline 10 & 30.002 & 75.01 & 0.47 & 49.66 & 0.19 \\
\hline 15 & 30.002 & 73.08 & 0.31 & 53.90 & 0.55 \\
\hline 20 & 30.002 & 72.11 & 0.49 & 58.65 & 0.34 \\
\hline 25 & 30.002 & 70.92 & 0.83 & 63.79 & 0.49 \\
\hline 30 & 30.002 & 69.32 & 0.45 & 68.24 & 0.51 \\
\hline 35 & 30.002 & 68.68 & 1.45 & 73.71 & 0.36 \\
\hline 10 & 34.997 & 74.20 & 0.13 & 55.06 & 0.04 \\
\hline 15 & 34.997 & 71.98 & 0.08 & 60.35 & 0.14 \\
\hline 20 & 34.997 & 70.64 & 0.00 & 65.92 & 0.06 \\
\hline 25 & 34.997 & 69.08 & 0.15 & 71.33 & 0.08 \\
\hline 30 & 34.997 & 68.44 & 1.01 & 76.57 & 0.90 \\
\hline 35 & 34.997 & 67.19 & 0.20 & 82.51 & 0.16 \\
\hline 10 & 38.274 & 73.29 & 0.14 & 59.26 & 0.21 \\
\hline 15 & 38.274 & 71.36 & 0.71 & 64.80 & 0.53 \\
\hline 20 & 38.274 & 70.17 & 0.42 & 69.82 & 0.13 \\
\hline 25 & 38.274 & 69.03 & 0.35 & 76.46 & 0.26 \\
\hline 30 & 38.274 & 67.62 & 0.52 & 82.06 & 0.86 \\
\hline 35 & 38.274 & 66.54 & 0.97 & 88.14 & 1.78 \\
\hline
\end{tabular}

Table 4. Means and standard deviations for complex dielectric constant measurements of seawater for each salinity and temperature. 
An examination of the six graphs shows that the GW data curves fall a little below the KS model results. To assess the amount more accurately, the \% differences are calculated in Table 5. The table values show that on the average the GW data is about $1.5 \%$ below the KS curves for the real part of the dielectric constant. The imaginary curves are overall about $2 \%$ below the KS data. The imaginary data does show an increasing error as the salinity and temperature increases. Looking at the individual curves, the KS curves appear very smooth since they are generated from algebraic expressions whose coefficients have been estimated from various data sources. The GW curves, on the other hand, are not as smooth since straight lines have been used to connect the mean measurement values. Regarding the standard deviations, it appears from examining all six graphs that the $\sigma$ increases with increasing temperature.

\subsubsection{Uncertainty in Seawater Measurements}

In this section, the uncertainty in the seawater measurements will be quantified. The uncertainty comes from three major sources:

- uncertainty in the seawater measurements themselves (Repeated measurements do not give exactly the same value of the complex dielectric constant each time they are performed.)

- uncertainty in the measurement of the calibration constant (There are 11 sets of acceptable measurements of methanol in Table 2. Each gives a slightly different value of the calibration constant, $C$.)

- uncertainty in the reference complex dielectric constant of methanol (The measurements reported by Gregory and Clarke (2009) have an average uncertainty of approximately 0.05 for the range of dielectric constants considered in this report.)

The cumulative effect of all these uncertainties will be computed. Since there are only a few data points for each measurement at a particular value of salinity and temperature, all measurements at the same temperature will be combined in making these calculations. An uncertainty will be found for the real and imaginary parts of the dielectric constants at each of the six temperatures measured.

Consider the uncertainty in the real dielectric constant first. The expression for the real dielectric constant from eq(2a) is:

$$
\varepsilon^{\prime}=2 C \Delta f / f_{o}
$$

The value of $\varepsilon^{\prime}$ can be considered a random variable that depends on the product of two random variables $C$ and $\Delta f$. The variation of $f_{o}$ is very small and can be neglected. If it is assumed that the random variables $C$ and $\Delta f$ are independent then the following expression is obtained for the mean of $\varepsilon^{\prime}$ :

$$
\left\langle\varepsilon^{\prime}\right\rangle=\frac{2\langle C\rangle\langle\Delta f\rangle}{f_{o}}
$$

The fluctuations for $\varepsilon^{\prime}$ can be expressed as $\delta \varepsilon^{\prime}=\varepsilon^{\prime}-\left\langle\varepsilon^{\prime}\right\rangle$. Now taking the fluctuations of eq(9) gives 

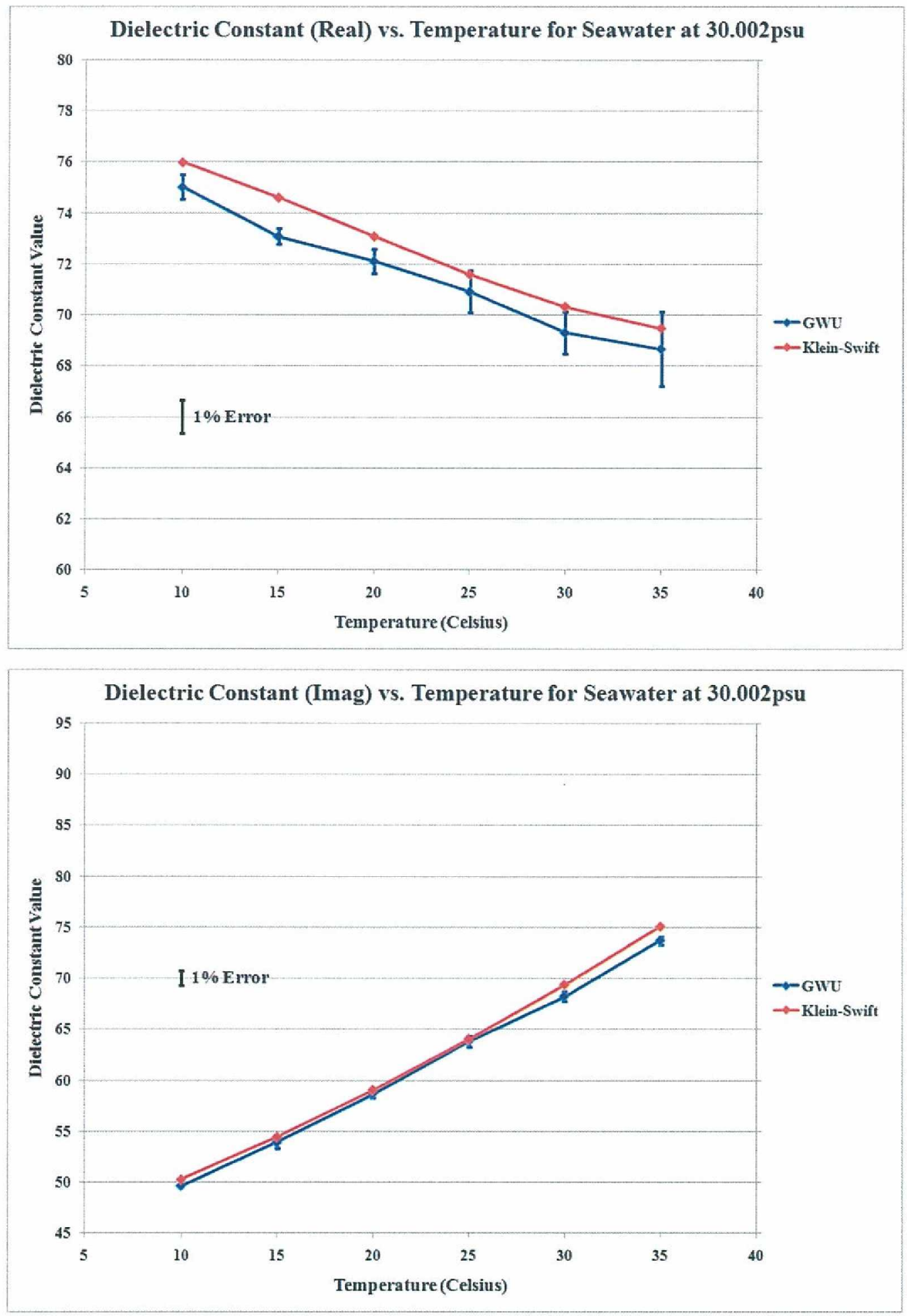

Figure 12. Real and imaginary dielectric constant of 30.002 psu seawater; GW results compared with KS model function. 

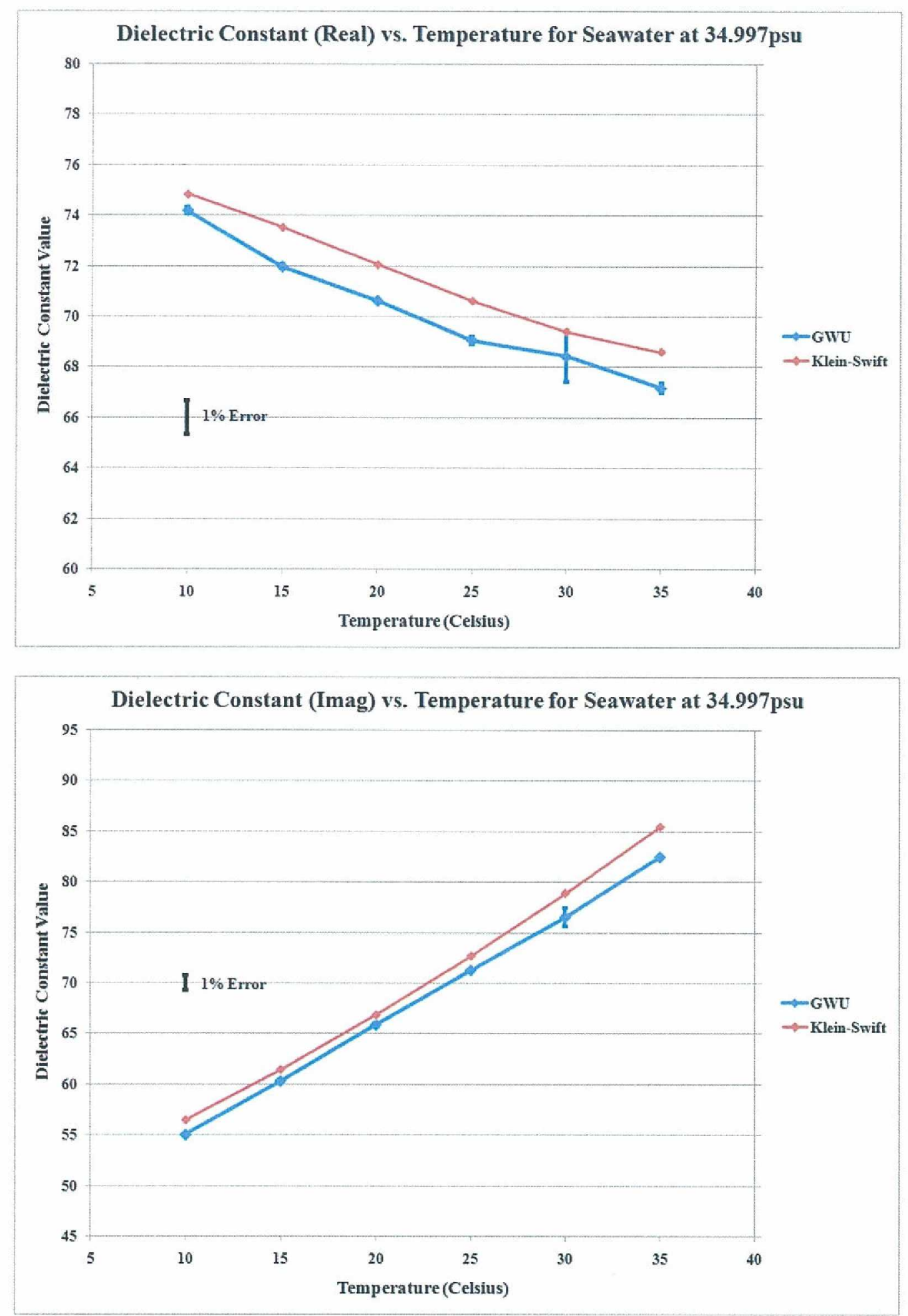

Figure 13. Real and imaginary dielectric constant of 34.999 psu seawater; GW results compared with $\mathrm{KS}$ model function. 

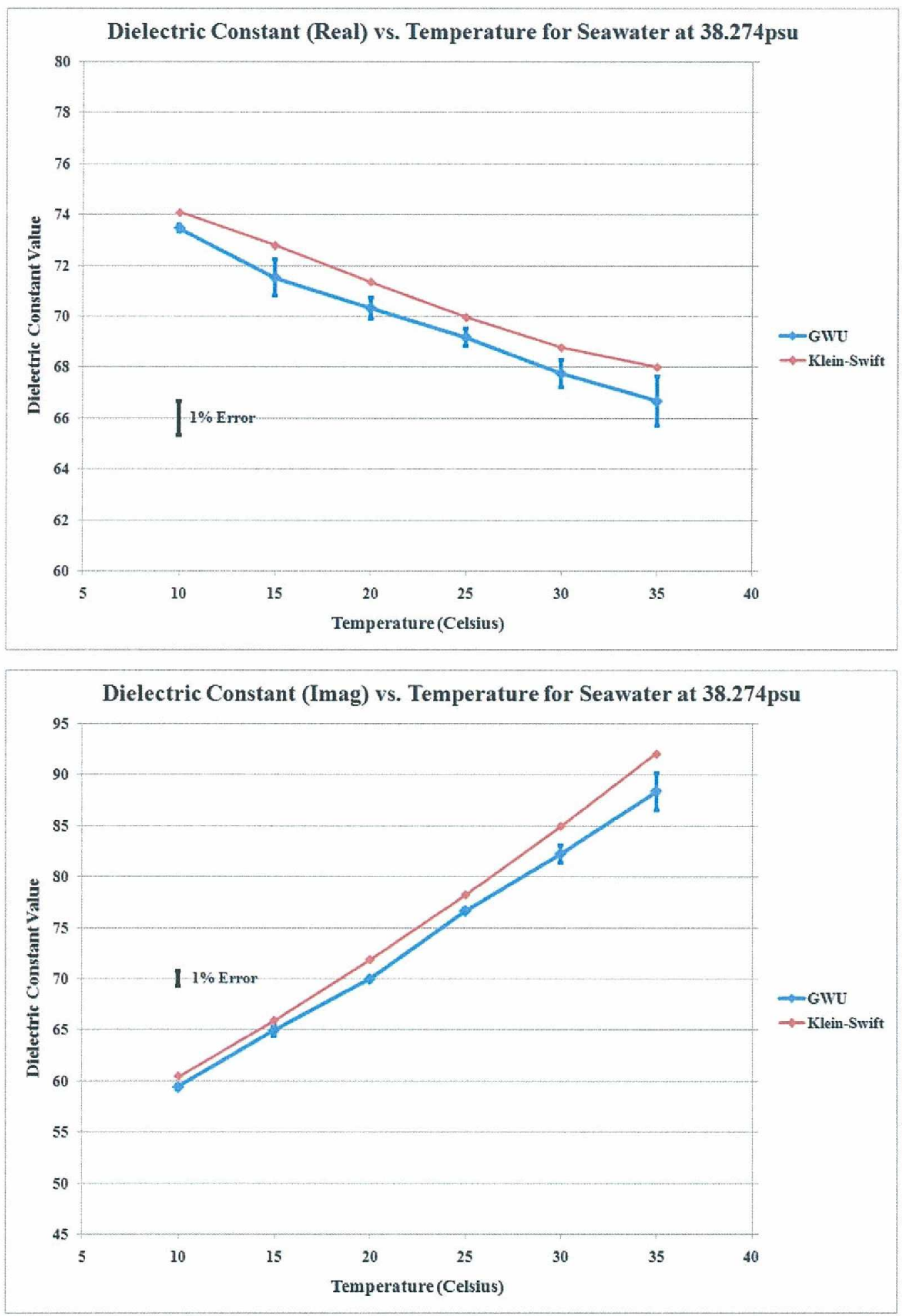

Figure 14. Real and imaginary dielectric constant of 38.274 psu seawater; GW results compared with KS model function. 


\begin{tabular}{|c|c|c|c|c|c|c|c|c|}
\hline \multicolumn{7}{|c|}{ Mean Measured Dielectric Constants vS. KS } \\
\hline & psu & Temp $^{\circ} \mathbf{C}$ & $\left\langle\varepsilon^{\prime}>\right.$ & $\varepsilon^{\prime}($ K\&S) & $\varepsilon^{\prime} \%$ diff & $<\varepsilon^{\prime \prime}>$ & $\varepsilon^{\prime \prime}($ KS) & $\varepsilon^{\prime \prime} \%$ diff \\
\hline & 30.002 & 10 & 75.01 & 75.97 & 1.28 & 49.66 & 50.26 & 1.21 \\
\hline & 30.002 & 15 & 73.08 & 74.60 & 2.08 & 53.90 & 54.43 & 0.98 \\
\hline & 30.002 & 20 & 72.11 & 73.07 & 1.33 & 58.65 & 59.03 & 0.65 \\
\hline & 30.002 & 25 & 70.92 & 71.58 & 0.93 & 63.79 & 64.02 & 0.36 \\
\hline & 30.002 & 30 & 69.32 & 70.33 & 1.46 & 68.24 & 69.38 & 1.67 \\
\hline & 30.002 & 35 & 68.68 & 69.48 & 1.16 & 73.71 & 75.10 & 1.89 \\
\hline Average & 30.002 & & & & 1.37 & & & 1.13 \\
\hline & 34.997 & 10 & 74.20 & 74.83 & 0.85 & 55.06 & 56.43 & 2.49 \\
\hline & 34.997 & 15 & 71.98 & 73.52 & 2.14 & 60.35 & 61.41 & 1.76 \\
\hline & 34.997 & 20 & 70.64 & 72.04 & 1.98 & 65.92 & 66.84 & 1.40 \\
\hline & 34.997 & 25 & 69.08 & 70.61 & 2.21 & 71.33 & 72.68 & 1.89 \\
\hline & 34.997 & 30 & 68.44 & 69.40 & 1.40 & 76.57 & 78.89 & 3.03 \\
\hline & 34.997 & 35 & 67.19 & 68.60 & 2.10 & 82.51 & 85.47 & 3.59 \\
\hline Average & 34.997 & & & & 1.78 & & & 2.36 \\
\hline & 38.274 & 10 & 73.29 & 74.07 & 1.06 & 59.26 & 60.41 & 1.94 \\
\hline & 38.274 & 15 & 71.36 & 72.79 & 2.00 & 64.80 & 65.90 & 1.70 \\
\hline & 38.274 & 20 & 70.17 & 71.35 & 1.68 & 69.82 & 71.86 & 2.92 \\
\hline & 38.274 & 25 & 69.03 & 69.95 & 1.33 & 76.46 & 78.23 & 2.31 \\
\hline & 38.274 & 30 & 67.62 & 68.78 & 1.72 & 82.06 & 84.99 & 3.57 \\
\hline & 38.274 & 35 & 66.54 & 68.01 & 2.21 & 88.14 & 92.11 & 4.50 \\
\hline & 38.274 & & & & 1.67 & & & 2.82 \\
\hline
\end{tabular}

Table 5. Real and imaginary mean dielectric constants; GW results compared with KS model values.

$$
\delta \varepsilon^{\prime}=\frac{2\langle C\rangle \delta(\Delta f)}{f_{o}}+\frac{2\langle\Delta f\rangle \delta C}{f_{o}}
$$

Examining eq(11) it is seen that the uncertainty in $\varepsilon^{\prime}$ is a result of the uncertainty in $\Delta f$ and in $\delta C$. Now the uncertainty in the calibration coefficient comes from two sources: the uncertainty in the methanol measurements and the uncertainty in the Gregory and Clarke's values. This can be expressed as

$$
\delta C=\delta C_{M}+\delta C_{r e f}
$$

where subscript $\mathrm{M}$ stands for methanol measurements and ref stands for reference measurements of Gregory and Clarke. Here, it is assumed that the methanol measurements made by GW are independent of the Gregory and Clarke measurements. Now putting eq(12) into eq(11) and taking the variance of the resulting equations gives

$$
\sigma_{\varepsilon^{\prime}}^{2}=\sigma_{\varepsilon^{\prime}}^{2}(S W)+\sigma_{\varepsilon^{\prime}}^{2}\left(C_{M}\right)+\sigma_{\varepsilon^{\prime}}^{2}\left(C_{r e f}\right)
$$

where 
$\sigma_{\varepsilon^{\prime}}(S W)=\frac{2\langle C\rangle \sigma_{\Delta f}}{f_{o}} \quad \sigma_{\varepsilon^{\prime}}\left(\mathrm{C}_{\mathrm{M}}\right)=\frac{2\langle\Delta f\rangle \sigma_{C_{M}}}{f_{o}} \quad \sigma_{\varepsilon^{\prime}}\left(\mathrm{C}_{r e f}\right)=\frac{2\langle\Delta f\rangle \sigma_{C_{r e f}}}{f_{o}}$

For a given salinity, the standard deviation of $\Delta f$ is computed separately for each temperature value and then the average of these standard deviations is found. This average is denoted by $\sigma_{\Delta f}$. The values of $\Delta f$ used in this calculation can be found in Appendix C.2. The quantity $\sigma_{C_{M}}$ is the standard deviation of the $C$ coefficients given in yellow in Table $3 \mathrm{a}$ and $\sigma_{C_{r f}}$ is the standard deviation of the $C$ constant with respect to an uncertainty of 0.05 in the reference measurements of methanol made by Gregory and Clarke.

\begin{tabular}{|c|c|c|c|c|}
\hline \multicolumn{5}{|c|}{ Real Dielectric Constant Uncertainty } \\
\hline Temp & $\sigma_{\varepsilon}(\mathbf{S W})$ & $\sigma_{\varepsilon}(\mathbf{C})$ & $\sigma_{\varepsilon}($ ref $)$ & $\sigma_{\varepsilon}$ (total) \\
\hline 10 & 0.25 & 0.56 & 0.14 & 0.62 \\
\hline 15 & 0.37 & 0.54 & 0.13 & 0.67 \\
\hline 20 & 0.3 & 0.53 & 0.13 & 0.63 \\
\hline 25 & 0.44 & 0.53 & 0.13 & 0.7 \\
\hline 30 & 0.66 & 0.53 & 0.12 & 0.86 \\
\hline 35 & 0.87 & 0.51 & 0.12 & 1.02 \\
\hline Average & & & & 0.75 \\
\hline
\end{tabular}

Table 6a. Uncertainty in the real part of the dielectric constant.

\begin{tabular}{|c|c|c|c|c|}
\hline \multicolumn{5}{|c|}{ Imaginary Dielectric Constant Uncertainty } \\
\hline Temp & $\sigma_{\varepsilon}(\mathbf{S W})$ & $\sigma_{\varepsilon}(\mathbf{C})$ & $\sigma_{\varepsilon}($ ref $)$ & $\sigma_{\varepsilon}$ (total) \\
\hline 10 & 0.15 & 0.41 & 0.1 & 0.44 \\
\hline 15 & 0.41 & 0.45 & 0.11 & 0.62 \\
\hline 20 & 0.17 & 0.48 & 0.12 & 0.53 \\
\hline 25 & 0.28 & 0.52 & 0.13 & 0.68 \\
\hline 30 & 0.76 & 0.58 & 0.14 & 1.03 \\
\hline 35 & 0.77 & 0.62 & 0.15 & 1.06 \\
\hline Average & & & & 0.73 \\
\hline
\end{tabular}

Table 6 b. Uncertainty in the imaginary part of the dielectric constant. 


\begin{tabular}{|c|c|c|c|c|c|}
\hline \multicolumn{5}{|c|}{ Percent Error in Real Dielectric Constant } \\
\hline Temp & $<\varepsilon^{\prime}>$ & $\%$ err( $\left.\varepsilon^{\prime}\right)$ & $\% \operatorname{err}(\mathbf{C})$ & $\%$ err(epsref) & \%err(total) \\
\hline 10 & 73.33 & 0.34 & 0.76 & 0.19 & 0.85 \\
\hline 15 & 71.12 & 0.51 & 0.76 & 0.19 & 0.94 \\
\hline 20 & 70.2 & 0.43 & 0.76 & 0.19 & 0.89 \\
\hline 25 & 69.14 & 0.64 & 0.76 & 0.19 & 1.01 \\
\hline 30 & 69.14 & 0.96 & 0.77 & 0.18 & 1.24 \\
\hline 35 & 66.41 & 1.31 & 0.76 & 0.19 & 1.53 \\
\hline Average & & & & & 1.08 \\
\hline
\end{tabular}

Table 7a. Total percent error in the real part and its components: variations in $\varepsilon^{\prime}$, in the calibration coefficient and in the reference dielectric.

\begin{tabular}{|c|c|c|c|c|c|}
\hline \multicolumn{5}{|c|}{ Percent Error in Imaginary Dielectric Constant } \\
\hline Temp & $<\varepsilon^{\prime \prime}>$ & $\%$ err( $\left.\varepsilon^{\prime \prime}\right)$ & $\%$ err(C) & \%err(epsref) & $\%$ err(total) \\
\hline 10 & 53.37 & 0.27 & 0.76 & 0.19 & 0.83 \\
\hline 15 & 59.56 & 0.69 & 0.76 & 0.19 & 1.04 \\
\hline 20 & 63.42 & 0.28 & 0.76 & 0.19 & 0.83 \\
\hline 25 & 67.79 & 0.41 & 0.76 & 0.19 & 1.01 \\
\hline 30 & 76.71 & 0.99 & 0.76 & 0.18 & 1.34 \\
\hline 35 & 81.83 & 0.94 & 0.76 & 0.19 & 1.3 \\
\hline Average & & & & & 1.06 \\
\hline
\end{tabular}

Table 7b. Total percent error in the imaginary part and its components: variations in $\varepsilon^{\prime \prime}$, in the calibration coefficient and in the reference dielectric.

Following a similar line of reasoning, $\left\langle\varepsilon^{\prime \prime}\right\rangle$ can be computed from eq(2b) by assuming that $\mathrm{C}$ and $\Delta(1 / Q)$ are independent random variables. The result is:

$$
\left\langle\varepsilon^{\prime \prime}\right\rangle=\langle C\rangle\langle\Delta(1 / Q)\rangle
$$

and the fluctuations of $\Delta(1 / Q)$ are given by

$$
\delta \Delta(1 / Q)=\langle C\rangle \delta \Delta(1 / Q)+\langle\Delta(1 / Q)\rangle \delta C
$$

Now following the reasoning used for the real part, the variance of the imaginary part of the dielectric constant, $\varepsilon^{\prime \prime}$, can be found. The result is: 


$$
\sigma_{\varepsilon^{\prime \prime}}^{2}=\sigma_{\varepsilon^{\prime \prime}}^{2}(S W)+\sigma_{\varepsilon^{\prime \prime}}^{2}\left(C_{M}\right)+\sigma_{\varepsilon^{\prime \prime}}^{2}\left(C_{r e f}\right)
$$

where

$$
\sigma_{\varepsilon^{n}}(S W)=\langle C\rangle \sigma_{\Delta Q} \quad \sigma_{\varepsilon^{n}}\left(\mathrm{C}_{\mathrm{M}}\right)=\langle\Delta(1 / \mathrm{Q})\rangle \sigma_{C_{M}} \quad \sigma_{\varepsilon^{n}}\left(\mathrm{C}_{r e f}\right)=\langle\Delta(1 / \mathrm{Q})\rangle \sigma_{C_{r e f}}
$$

For a given salinity, the standard deviation of $\Delta(1 / \mathrm{Q})$ is computed separately for each temperature value and then the average of these standard deviations is found. This average is denoted by $\sigma_{\Delta Q}$. The values of $\Delta(1 / \mathrm{Q})$ used in this calculation can be found in Appendix C.2.

An examination of the uncertainties, shown in Tables $6 \mathrm{a}$ and $6 \mathrm{~b}$, point out that the values tend to increase with temperature but the increase is not dramatic. It demonstrates that the uncertainty, due to the seawater measurement, increases with temperature. The values in the tables show that the contribution of variations in the reference measurements is small. There also does not appear to be major differences between the behavior of the uncertainty of the real and imaginary parts. The values appearing in Table $6 \mathrm{a}$ can be put in terms of percent error by dividing the standard deviations by $\left\langle\varepsilon^{\prime}\right\rangle$ and multiplying by 100 . The results are shown in Table 7a. The value of $\left\langle\varepsilon^{\prime}\right\rangle$ at each temperature is computed by using eq(10) and averaging $\Delta f$ over all measurements with that temperature. The percent error of the imaginary part is computed in a similar way by using eq(15) to compute $\left\langle\varepsilon^{\prime \prime}\right\rangle$ at each temperature. The percent errors for the imaginary part are shown in Table $7 \mathrm{~b}$. Both tables show that the measurement results, after taking all major sources of error into account, vary about $1 \%$ of their mean value. It is observed that the calibration measurements of methanol made by GW account for about three quarters of the total variation.

\section{Conclusions}

Seawater measurements were made at 30,35 and $38 \mathrm{psu}$ for a temperature range of $10^{\circ} \mathrm{C}$ to $35^{\circ} \mathrm{C}$ in $5^{\circ} \mathrm{C}$ intervals. Several measurements were taken at each salinity and temperature. From these measurements, a mean and a standard deviation were calculated. The standard deviation of the measurements was lowest at low temperatures (approximately $0.3 \%$ ) while it was highest at high temperatures (approximately 1.0\%). In addition to measurement variations, there were variations in measurements used to calculate the calibration coefficient. These measurements were taken using methanol as a reference liquid at a temperature of $20^{\circ} \mathrm{C}$. It was observed that variations in the methanol measurements were a substantial contributor to the overall variance of the measurement data. Total measurement variation in both the real and imaginary parts due to changes in the seawater measurements, in the methanol measurements and in the reference value of methanol was less than or equal to approximately $1.0 \%$ for temperatures of $25^{\circ} \mathrm{C}$ or less. For $30^{\circ} \mathrm{C}$ and $35^{\circ} \mathrm{C}$, the variation was about $1.3 \%$. 
Comparisons of these dielectric measurements with the predictions of the KS model function (Klein and Swift 1977) have been discussed. The GW data for the real part of the dielectric constant is about $1.5 \%$ below the Klein and Swift data. The difference does not appear to be a function of temperature. At present there seems no simple explanation for this difference. The imaginary part of the dielectric constant is on the average about $2.0 \%$ below the Klein and Swift results but the difference tends to increase with both temperature and salinity. This difference may be a result of the microwave heating that occurred in the Ho et al data. This heating increases with seawater conductivity that, in turn, increases with temperature and salinity.

In the future measurements attempts will be made to reduce the measurement variations. One option is to position the matching networks within the coolant liquid to stabilize their temperature. A slightly larger tube radius could be used to increase the frequency difference between empty and sample measurements. Assuming that the noise remains the same, this would improve the accuracy. The measurements of the calibration liquid can be improved by making them at a lower temperature. An examination of the seawater measurements shows that the uncertainty diminishes with decreasing temperatures. It is conjectured that the same would be true when measuring methanol. In the future, we hope to measure other salinities and to measure all salinities at lower temperatures.

\section{References}

Bacon, S., F. Culkin, N. Higgs and P. Rideout (2007), "IAPSO standard seawater: definition of the uncertainty in the calibration, procedure and stability of recent batches," J. Atm and Ocean Technology, Vol. 24, No. 10.

Baker-Jarvis, J., R.G. Geyer, J.H. Grovenor, M.D. Janezic, C.A. Jones B. Riddle, C. M. Weil and J. Krupka (1998), "Dielectric Characterization of Low-Loss Materials - A Comparison of Techniques," IEEE Trans Diel. and Elect. Insul., Vol. 5, pp. 571-577.

Baker-Jarvis, J., M.D. Janezic, B. Riddle, C.L. Holloway, N.G. Paulter and J.E. Blendell (2001), "Dielectric and conductor-loss characterization and measurements on electronic packaging materials," NIST Technical Note 1520, 19.

Blue, M.D.(1980), "Permittivity of Ice and Water at Millimeter Wavelengths," J. of Geophyical Res., Vol 85, C2, pp. 1101-1106.

Blume, H-J. C., B.M. Kendall and J.C. Fedors (1978), "Measurements of Ocean Temperature and Salinity via Microwave Radiometry," Bound. Layer Meteorol., Vol 13, pp 295-308.

Blanch, S. and A. Aguasca (2004), "Seawater dielectric permittivity model from measurements at L band of seas water at L band, "Proceedings of IGARSS 2004, pp. 1362-1365.

Buckmaster, H.A. (1990), "Precision microwave complex permittivity measurements of high loss liquids," J Electromagnetic Waves and Appl., Vol. 4, pp. 645-659. 
Chen,L.F., C.K. Ong, V.V. Varadan and V.K. Varadan (2004), Microwave Electronics, Chapter 6, Publisher Wiley.

Dinnat, E.,J. Boutin, G. Cardal and J. Etcheto (2003), "Issues concerning the sea emissivity modeling at L band for retrieving surface salinity," Radio Science, Vol 38, No 4, doi: $10.1029 / 2002$ RS002637.

Ellison, W., A. Balana, G. Delbos, K. Lamkaouchi, L. Eymard, C. Guillou and C. Prigent (1996), "Study and measurement of the dielectric properties of seawater," Final Report, ESTEC/ESA Contr. 11197/94/NL/CN.

Ellison, W., A. Balana, G. Delbos, K. Lamkaouchi, L. Eymard, C. Guillou and C. Prigent (1998), "New permittivity measurements of seawater," Radio Science, Vol. 33, pp. 639-648.

Estin, A.J. and H.E. Bussey (1960), "Errors in dielectric measurements due to a sample insertion hole in a cavity," IRE Trans. Microwave Theory and tech., Vol 8 p. 650.

Forsch, C., M. Knudsen and S.P. Sorensen, (1902), "Reports on the determination of the Constants for compilation of hydrographic tables," Kgl. Dan. Vidensk. Selsk, Skifier, 6 Raekke Naturvidensk Mat, Copenhagen, Vol.12(1), pp. 1-151.

Goodberlet, M.A., et al, (1997), "Microwave Remote Sensing of Coastal Zone Salinity", J. Coastal Res., Vol 13, pp 363-372.

Gregory, A.P. and R.N. Clarke (2009), "Tables of the Complex Permittivity of Dielectric reference Liquids at frequencies up to $5 \mathrm{GHz}$," National Physical Laboratory Report NPL Report MAT 23, Middlesex, UK

Ho, W.A., and W.F. Hall (1973), "Measurements of the dielectric properties of seawater of seawater and $\mathrm{NaCl}$ solutionsat $2.65 \mathrm{GHz}$, “J. Geophys. Res., Vol. 78, pp. 6301-6315.

Ho, W.A., W. Love and M.J. van Melle (1974) "Measurements of the dielectric properties of sea water at $1.43 \mathrm{GHz}, "$ NASA Contract Rep., CR-2458.

Janiczek, J.(2007) "Design of a Temperature and Microwave Control and Information System for Seawater Analysis," Senior Design Project Final Report, Dept. of ECE, George Washington University, Washington, DC.

Jenkins, S., T.E. Hodgetts, R.N. Clark and A.W. Preece (1990), "Dielectric measurements on reference liquids using automatic network analyzers and calculable geometries," Meas. Sci. Technol., Vol. 1, pp. 691-702.

Kaatze, U., (1989), "Complex Permittivity of Water as a Function of Frequency and Temperature," J. Chem. Eng. Data, Vol 34, pp. 371-374. 
Kajfez,D., S. Chebolu, M.R. Adul-Gaffoor and A.A. Kishk (1999), "Uncertainty Analysis of the Transmission Type Measurement of Q-Factor," IEEE Trans. Microwave Theory and Tech. Vol. 47, pp.367-371.

Klein, L. and C. Swift (1977), "An improved model for the dielectric constant of seawater at microwave frequencies, "IEEE TAP, Vol. 25, pp. 104-111.

Koblinsky, C.J., P. Hildebrand, D. Le Vine, et al, (2003), "Sea Surface Salinity from Space: Science Goals and measurement Approach", Radio Sci., Vol 38 (No. 4), Mar 29-1 to 29-7

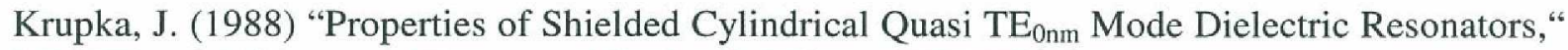
IEEE Trans. Microwave Theory and Tech., Vol 36, pp.774-778.

Lagerloef, G., C. Swift, D. Le Vine, (1995), "Sea Surface Salinity: The Next Remote Sensing Challenge", Oceanogr. Vol 8, pp 44-50.

Lane, J. and J. Saxon (1952), "Dielectric dispersion in pure polar liquids at very high frequencies, "Proc. Roy. Soc., Vol. A213, pp. 531-545.

Lang, R.H., C. Utku and D.M. Le Vine (2003), "Measurement of the dielectric constant of seawater at L-band," IGARSS2003, Vol I, pp.19-21.

Lang, R.H, C, Utku, J, Janiczek, Y. Tarkocin and D.M. Le Vine (2007), "Accurate L-Band Measurements of the Dielectric Constant of Seawater, "Proc IGARSS 2007 pp.5255-5257.

Lang, R.H., Y. Tarkocin, C. Utku and D.M. le Vine (2008a), "Accurate L-Band Dielectric Constant Measurements of Seawater, “ Proc. IEEE MicroRad 2008, pp. 1-4.

Lang, R.H., Y. Tarkocin, C. Utku and D.M. Le Vine (2008b), "Recent results on the accurate measurements of the dielectric constant of seawater at $1.413 \mathrm{GHz}$," Proc. IGARSS 2008, Vol IV, pp.950-953.

Le Vine, D.M., M. Kao, R. Garvine and T. Sanders (1998), "Remote Sensing of Ocean Salinity: Results from the Delaware Coastal Current Experiment", J. Atmos. Oceanic Tech, Vol 15, pp 1478-1484.

Lewis, E.L. (1980), "The Priactical Saliniyt Scale 1978 and Its Antecedents", J. Oceanic Engin, Vol 5 (No. 1), pp3-8.

Lewis, E.L. and R.G. Perkin (1981), "The Practical Salinity Scale 1978: Conversion of Existing Data", Deep-Sea Res., Vol 28A (No. 4), pp 307-328.

Meissner, T. and F. Wentz, (2004), "The Complex Dielectric Constant of Pure and Sea Water from SSM/I, TMI and AMSR Brightness Temperature Observations", Proc. Specialist Meeting on Microwave Radiometry and Remote Sensing Applications, Rome, Italy, February 
Montgomery, C.G. (1947) Techniques of Microwave Measurements, Chapter Ch. 10, McGraw Hill, New York, NY.

Nicolson, A.M. and G.F. Ross (1970) "Measurements of the intrinsic properties of materials by time-domain techniques, “IEEE Trans. Instrum. Meas., IEEE IM Vol.19, pp. 377-82.

Nyshadham, A., S.L. Christopher and S.S. Stuchly (1992), "Permittivity measurements using open-ended sensors and reference liquid calibration - an uncertainty analysis," IEEE MTT, Vol. 40 , pp. 305-314.

Roberts, S. and A. von Hippel (1946),"A new method for measuring dielectric constant and loss in the range of centimeter waves, "J. Appl. Phys., Vol. 17, pp. 610-616.

Saxton, J.A. and J.A. Lane (1952), "Electrical Properties of Seawater," Wireless Eng., vol. 29, pp. 269-275

Sivestrin, J. P., M. Berger, Y. Kerr and J. Font, (2001), "ESA's Second Earth Explorer Opportunity Mission: The Soil Moisture and Ocean Salinity Mission - SMOS", IEEE Geoscience and Remote Sensing Newsletter, Vol. 118, pp 11-14

Stogryn, A. (1971) "Equations for calculating the dielectric constant of saline water," IEEE MTT, Vol. 19, pp. 733-736.

Stogryn, A., H.T. Bull, K. Rubayi and S. Iravanchy (1995), "The microwave permittivity of seas and fresh water," Aerojet Report, Azusa, CA

Swift, C (2006) private communication.

Sucher, M. (1963) Handbook of Microwave Measurements, Edited by H.M. Altschuler, Chapter VIII, Measurement of Q, Publisher: Polytechnic Institute of Brooklyn, Brooklyn, NY 


\section{Appendix A \\ Sensitivity of Salinity to Variations in the Model Function}

The following discussion addresses the issue of how accurate the dielectric measurements of seawater have to be in order to obtain a given level of uncertainty in the salinity measurements from space. Since these dielectric measurements will be used to construct a model function, the model function will be as accurate as these measurements .

To pursue this issue, we start by developing the dependence of the brightness temperature as a function of the temperature and the salinity. Consider the relationship between brightness temperature and dielectric constant of seawater as given in eq(1a) and eq(1b) for the case of a radiometer looking directly down, normal to a flat sea surface. By using the equation for the model function (eq(1c)) along with eq(1a) and eq(1b) we obtain an expression for the brightness temperature in terms of the temperature and the salinity as shown below:

$$
T_{B}=T_{B}(\varepsilon(T, S), T)
$$

Although this relationship has been obtained for a downward pointing radiometer on a flat ocean surface, it is valid in much more general conditions.

It is assumed that the exact model function, $\varepsilon(S, T)$, is unknown. An approximate model function, $\varepsilon_{m}(S, T)$, is known. Now if $T_{B o}$ is the observed brightness temperature for seawater at salinity $S_{o}$ and temperature $T_{o}$, then:

$$
T_{B o}=T_{B}\left(\varepsilon\left(T_{o}, S_{o}\right), T_{o}\right)
$$

Assuming $T_{o}$ is known exactly and since $\varepsilon_{m}$ is known instead of $\varepsilon$ then we naturally understand the above equation as:

$$
T_{B o}=T_{B}\left(\varepsilon_{m}\left(T_{o}, S_{m}\right), T_{o}\right)
$$

for some value of salinity $S_{m}$.

The question that naturally arises is: How different are $S_{o}$ and $S_{m}$ ? A quantitative estimate for this difference can be obtained from eq( $1 \mathrm{~A})$ and eq( $2 \mathrm{~A})$, since both correspond to the same observed brightness temperature. Hence one has:

$$
T_{B}\left(\varepsilon\left(T_{o}, S_{o}\right), T_{o}\right)=T_{B}\left(\varepsilon_{m}\left(T_{o}, S_{m}\right), T_{o}\right) .
$$

Now define the errors $\delta \varepsilon_{m}$ and $\delta S_{m}$ through $\varepsilon=\varepsilon_{m}+\delta \varepsilon_{m}$ and $S_{m}=S_{o}+\delta S_{m}$ respectively. Note that with these definitions $\delta \varepsilon_{m}$ corresponds to the modeling error in $\varepsilon$ and $\delta S_{m}$ corresponds to the error in retrieved salinity

Equation (3A) can now be expressed as: 


$$
T_{B}\left(\varepsilon_{m}\left(T_{o}, S_{o}\right)+\delta \varepsilon_{m}, T_{o}\right)=T_{B}\left(\varepsilon_{m}\left(T_{o}, S_{o}+\delta S_{m}\right), T_{o}\right) .
$$

Expand both sides to a Taylor series in the relevant quantity and assume $\partial\left(\delta \varepsilon_{m}\right) / \partial S$ can be neglected. The expanded equation is:

$$
\begin{aligned}
& T_{B}\left(\varepsilon_{m}\left(T_{o}, S_{o}\right), T_{o}\right)+\frac{\partial T_{B}}{\partial \varepsilon_{m}^{\prime}} \delta \varepsilon_{m}^{\prime}+\frac{\partial T_{B}}{\partial \varepsilon_{m}^{\prime \prime}} \delta \varepsilon_{m}^{\prime \prime}= \\
& T_{B}\left(\varepsilon_{m}\left(T_{o}, S_{o}\right), T_{o}\right)+\left[\frac{\partial T_{B}}{\partial \varepsilon_{m}^{\prime}} \frac{\partial \varepsilon_{m}^{\prime}}{\partial S}+\frac{\partial T_{B}}{\partial \varepsilon_{m}^{\prime \prime}} \frac{\partial \varepsilon_{m}^{\prime \prime}}{\partial S}\right] \delta S_{m}
\end{aligned}
$$

The above expression then yields:

$$
\frac{\partial T_{B}}{\partial \varepsilon_{m}^{\prime}} \delta \varepsilon_{m}^{\prime}+\frac{\partial T_{B}}{\partial \varepsilon_{m}^{\prime \prime}} \delta \varepsilon_{m}^{\prime \prime}=\left[\frac{\partial T_{B}}{\partial \varepsilon_{m}^{\prime}} \frac{\partial \varepsilon_{m}^{\prime}}{\partial S}+\frac{\partial T_{B}}{\partial \varepsilon_{m}^{\prime \prime}} \frac{\partial \varepsilon_{m}^{\prime \prime}}{\partial S}\right] \delta S_{m}
$$

Hence:

$$
\delta S_{m}=\frac{\frac{\partial T_{B}}{\partial \varepsilon_{m}^{\prime}} \delta \varepsilon_{m}^{\prime}+\frac{\partial T_{B}}{\partial \varepsilon_{m}^{\prime \prime}} \delta \varepsilon_{m}^{\prime \prime}}{\frac{\partial T_{B}}{\partial \varepsilon_{m}^{\prime}} \frac{\partial \varepsilon_{m}^{\prime}}{\partial S}+\frac{\partial T_{B}}{\partial \varepsilon_{m}^{\prime \prime}} \frac{\partial \varepsilon_{m}^{\prime \prime}}{\partial S}}
$$

Equations (1) and (2) can be used to calculate the following derivatives $\left(\varepsilon_{m}^{\prime}>>1\right)$ :

$$
\begin{aligned}
& \frac{\partial T_{B}}{\partial \varepsilon_{m}^{\prime}} \approx 2 T|\Gamma|^{2} \operatorname{Re}\left\{\varepsilon_{m}^{-3 / 2}\right\} \\
& \frac{\partial T_{B}}{\partial \varepsilon_{m}^{\prime \prime}} \approx 2 T|\Gamma|^{2} \operatorname{Im}\left\{\varepsilon_{m}^{-3 / 2}\right\}
\end{aligned}
$$

Inserting the above expressions for the derivatives in eq(4A) yields the error in the retrieved salinity as:

$$
\delta S_{m}=\frac{\operatorname{Re}\left\{\varepsilon_{m}^{-3 / 2}\right\} \delta \varepsilon_{m}^{\prime}+\operatorname{Im}\left\{\varepsilon_{m}^{-3 / 2}\right\} \delta \varepsilon_{m}^{\prime \prime}}{\operatorname{Re}\left\{\varepsilon_{m}^{-3 / 2}\right\} \frac{\partial \varepsilon_{m}^{\prime}}{\partial S}+\operatorname{Im}\left\{\varepsilon_{m}^{-3 / 2}\right\} \frac{\partial \varepsilon_{m}^{\prime \prime}}{\partial S}}
$$

A worst case error bound is:

$$
\left|\delta S_{m}\right| \leq \frac{\left|\operatorname{Re}\left\{\varepsilon_{m}^{-3 / 2}\right\} \| \delta \varepsilon_{m}^{\prime}\right|+\left|\operatorname{Im}\left\{\varepsilon_{m}^{-3 / 2}\right\}\right|\left|\delta \varepsilon_{m}^{\prime \prime}\right|}{\left|\operatorname{Re}\left\{\varepsilon_{m}^{-3 / 2}\right\} \frac{\partial \varepsilon_{m}^{\prime}}{\partial S}+\operatorname{Im}\left\{\varepsilon_{m}^{-3 / 2}\right\} \frac{\partial \varepsilon_{m}^{\prime \prime}}{\partial S}\right|}
$$


The Klein/Swift model can used to obtain an estimate of the derivatives $\partial \varepsilon_{m}^{\prime} / \partial S$ and $\partial \varepsilon_{m}^{\prime \prime} / \partial S$. Figure 2 (a) shows the percentage error in retrieved salinity $\left|\delta S_{m}\right|$ as a function of salinity at various temperatures for 0.2 modeling error in both the real and imaginary parts. Figures 2 (b and c) show that an error in the real part has a larger effect than an error in the imaginary part. Although the expression in eq(A5) has been obtained for a special case, the result can to generalized to treat oblique observation angles and polarization effects.

\section{Appendix B}

\section{Derivation of the Perturbations Equations for the Measurement of Dielectric Constants}

In determining the sample dielectric permittivity using the perturbation technique, uncertainties in field quantities established inside the cavity measurement system demand certain approximations to be made to these quantities. These approximations, combined with the uncertainties in the dimensions of the measurement system, reduce the accuracy of the retrieved sample permittivity. Nevertheless the adverse effects of these uncertainties can be mitigated by proper calibration. A common calibration procedure involves the use of a reference liquid of known dielectric permittivity thus eliminating the need to accurately predict the fields inside the cavity as well as the precise knowledge of cavity system dimensions. This calibration procedure however has two inherent drawbacks albeit to lesser extent. These drawbacks are (i) the accuracy of the calibration is limited by the accuracy of reference liquid dielectric permittivity and (ii) the measurement system response is slightly different for the reference liquid and the sample. In this section we investigate the accuracy of the perturbation method when used in conjunction with the reference liquid calibration procedure.

Consider a cylindrical cavity having a height $l$ and radius $a_{3}$ with an empty sample tube placed along its axis. The cavity is of the transmission type and the sample tube has a very small inner radius $a_{1}$ and an outer radius $a_{2}$. This inner cylindrical region inside the sample tube where the liquid sample can be introduced subtends a volume $V_{o}$. Without the liquid sample, the dielectric constant inside the cavity is equal to $\varepsilon_{o}$ i.e. that of air except for the sample tube whose relative dielectric constant is real and is denoted by $\varepsilon_{t}$. The relative dielectric profile for the empty cavity is denoted by the real profile function $\varepsilon_{r e l}(r)$ where $r$ is a radial coordinate.

The cavity is excited through an aperture $A_{1}$ and the output power is withdrawn by a second aperture $A_{2}$. It is assumed that the empty cavity has only wall and end losses and the cavity resonates at the radial frequency $\omega_{o}$. In this case it follows from the complex Poynting theorem that:

$$
\int_{S} \mathfrak{R e}\left\{\boldsymbol{E}_{o} \times \boldsymbol{H}_{o}^{*}\right\} \cdot d s=0
$$


where $\left\{\boldsymbol{E}_{o}, \boldsymbol{H}_{o}\right\}$ are the fields inside the empty cavity and $S$ is the inner surface of the cavity with unit normal $\hat{n}$ and $d s=\hat{n} d s$ is the vector differential surface element. Note that the normal points out of the cavity. If we denote the total aperture surface by $A=A_{1} \cup A_{2}$ and the cavity walls and ends by $S-A$ i.e. the cavity inner surface minus the total aperture opening, then eq(1) can be expressed as:

$$
\int_{S=A} \Re \mathrm{e}\left\{\boldsymbol{E}_{o} \times \boldsymbol{H}_{o}^{*}\right\} \cdot d \boldsymbol{s}=-\int_{A} \Re \mathrm{Re}\left\{\boldsymbol{E}_{o} \times \boldsymbol{H}_{o}^{*}\right\} \cdot d \boldsymbol{s}
$$

This equation expresses the fact that the real power input to the cavity minus the output from the cavity through the apertures is dissipated by the lossy walls and ends of the cavity.

Next consider the cavity with the sample liquid introduced into the sample tube. In this case, the relative dielectric profile is the same everywhere except inside the sample tube where the relative dielectric constant is now equal to the complex relative dielectric constant of the sample i.e. $\varepsilon_{s}=\varepsilon_{s}^{\prime}+i \varepsilon_{s}^{\prime \prime}$. This new relative dielectric profile will be denoted by $\tilde{\varepsilon}_{r e l}(r)$. Hence with this notation, $\widetilde{\varepsilon}_{r e l}=\varepsilon_{s}$ in $V_{o}$ and $\widetilde{\varepsilon}_{r e l}=\varepsilon_{r e l}$ outside $V_{o}$. If the fields inside the cavity with the sample are now denoted by the pair $\{\boldsymbol{E}, \boldsymbol{H}\}$, then again by the complex Poynting theorem it follows that:

$$
\int_{S-A} \Re\left(\left\{\boldsymbol{E} \times \boldsymbol{H}^{*}\right\} \cdot d s+\omega \varepsilon_{o} \varepsilon_{s}^{\prime \prime} \int_{V_{o}} \boldsymbol{E} \cdot \boldsymbol{E}^{*} d v=-\int_{\boldsymbol{A}} \Re \mathrm{e}\left\{\boldsymbol{E} \times \boldsymbol{H}^{*}\right\} \cdot d s\right.
$$

where $\omega$ is the resonance frequency of the cavity with the sample. Note that $\varepsilon_{s}^{\prime \prime}$ is the imaginary part of the sample relative dielectric constant at $\omega$ and $V_{o}$ is the volume of the sample. The above equation now expresses the fact that the real power input to the cavity through the aperture is dissipated by the lossy walls of the cavity and the loss in the sample.

To a very good approximation we can assume that the wall losses of the cavity do not change when the small amount of sample liquid is introduced into the cavity. Formally we can write:

$$
\int_{S \cdot A} \Re \mathrm{e}\left\{\boldsymbol{E} \times \boldsymbol{H}^{*}\right\} \cdot d s \approx \int_{S \cdot A} \Re \mathrm{e}\left\{\boldsymbol{E}_{o} \times \boldsymbol{H}_{o}^{*}\right\} \cdot d s
$$

Then using this approximation in eq(B3) along with eq(B2) leads to the result:

$$
\omega \varepsilon_{o} \varepsilon_{s}^{\prime \prime} \int_{V_{o}} \boldsymbol{E} \cdot \boldsymbol{E}^{*} d v=-\left\{\int_{\boldsymbol{A}} \Re \mathrm{R}\left\{\boldsymbol{E} \times \boldsymbol{H}^{*}\right\} \cdot d \boldsymbol{s}-\int_{A} \Re \mathrm{R}\left\{\boldsymbol{E}_{o} \times \boldsymbol{H}_{o}^{*}\right\} \cdot d \boldsymbol{s}\right\}
$$

implying that the additional real power input to the cavity after the sample is introduced, compensates the loss in the sample. 
Let us now introduce the perturbation fields $\boldsymbol{e}=\boldsymbol{E}-\boldsymbol{E}_{0}$ and $\boldsymbol{h}=\boldsymbol{H}-\boldsymbol{H}_{0}$, the norms of which are small compared to the norms of $\boldsymbol{E}_{o}$ and $\boldsymbol{H}_{o}$. We can then rewrite (B4) as:

$$
\omega \varepsilon_{o} \varepsilon_{s}^{\prime \prime} \int_{V_{o}} \boldsymbol{E} \cdot \boldsymbol{E}^{*} d v \approx-\int_{A} \Re \mathrm{e}\left\{\boldsymbol{E}_{o} \times \boldsymbol{h}^{*}+\boldsymbol{e} \times \boldsymbol{H}_{o}^{*}\right\} \cdot d s
$$

where we have ignored the second order term $e \times h^{*}$.

Now let us obtain relations for retrieving the dielectric constant of the sample. For this purpose we make use of the vector expression:

$$
\nabla \cdot\left(\boldsymbol{E}_{o}^{*} \times \boldsymbol{H}+\boldsymbol{E} \times \boldsymbol{H}_{o}^{*}\right)=i\left(\omega-\omega_{o}\right) \mu_{o} \boldsymbol{H} \cdot \boldsymbol{H}_{o}^{*}+i\left(\omega \tilde{\varepsilon}_{r e l} \boldsymbol{E} \cdot \boldsymbol{E}_{o}^{*}-\omega_{o} \varepsilon_{r e l} \boldsymbol{E} \cdot \boldsymbol{E}_{o}^{*}\right)
$$

where $\mu_{o}$ is the magnetic permeability of air. Using the divergence theorem in eq(B6) and rearranging yields:

$$
\begin{aligned}
\int_{S}\left(\boldsymbol{E}_{o}^{*} \times \boldsymbol{H}+\boldsymbol{E} \times \boldsymbol{H}_{o}^{*}\right) \cdot d s+\omega \varepsilon_{o} \varepsilon_{s}^{\prime \prime} \int_{V_{o}} \boldsymbol{E} \cdot \boldsymbol{E}_{o}^{*} d v & =i \omega \varepsilon_{o} \Delta \varepsilon \int_{V_{o}} \boldsymbol{E} \cdot \boldsymbol{E}_{o}^{*} d v \\
& -i \Delta \omega \int_{V}\left(\varepsilon_{o} \varepsilon_{r e l} \boldsymbol{E} \cdot \boldsymbol{E}_{o}^{*}+\mu_{o} \boldsymbol{H} \cdot \boldsymbol{H}_{o}^{*}\right) d v
\end{aligned}
$$

where $\Delta \omega=\omega_{o}-\omega$ and $\Delta \varepsilon=\varepsilon_{s}^{\prime}-1$. If we now consider the fact that the perturbation fields are small, then we can neglect the imaginary parts of all the integrands in the above. Consequently, taking the real and imaginary parts of the above yields the following relations respectively:

$$
\int_{S} \Re \mathrm{Re}\left\{\boldsymbol{E}_{o}^{*} \times \boldsymbol{H}+\boldsymbol{E} \times \boldsymbol{H}_{o}^{*}\right\} \cdot d s+\omega \varepsilon_{o} \varepsilon_{s}^{\prime \prime} \int_{V_{o}} \mathfrak{R e}\left\{\boldsymbol{E} \cdot \boldsymbol{E}_{o}^{*}\right\} d v=0
$$

and

$$
\frac{\Delta \omega}{\omega}=\frac{\varepsilon_{o} \Delta \varepsilon \int_{V_{o}} \Re \mathrm{e}\left\{\boldsymbol{E} \cdot \boldsymbol{E}_{o}^{*}\right\} d v}{\int_{V} \Re \mathrm{e}\left\{\varepsilon_{o} \varepsilon_{r e l} \boldsymbol{E} \cdot \boldsymbol{E}_{o}^{*}+\mu_{o} \boldsymbol{H} \cdot \boldsymbol{H}_{o}^{*}\right\} d v}
$$

Equation (B8) is a common relation in literature. The real part of the relative dielectric constant for the sample can be found by inverting eq(B8) as:

$$
\Delta \varepsilon=2 C \frac{\Delta \omega}{\omega}
$$

and 


$$
C=\frac{1}{2} \frac{\int_{V} \Re \mathrm{e}\left\{\varepsilon_{o} \varepsilon_{r e l} \boldsymbol{E} \cdot \boldsymbol{E}_{o}^{*}+\mu_{o} \boldsymbol{H} \cdot \boldsymbol{H}_{o}^{*}\right\} d v}{\varepsilon_{o} \int_{V_{o}} \mathfrak{R e}\left\{\boldsymbol{E} \cdot \boldsymbol{E}_{o}^{*}\right\} d v}
$$

The constant $C$ is dependent on the dielectric constant of the sample itself. We can write it as the product of a term that depends only the empty cavity features and a term that is also dependent on the sample dielectric. For this purpose we make use of the perturbation fields to obtain:

$$
C=\frac{1}{2} \frac{\int_{V} \operatorname{Re}\left\{\varepsilon_{o} \varepsilon_{r e l} \boldsymbol{E} \cdot \boldsymbol{E}_{o}^{*}+\mu_{o} \boldsymbol{H} \cdot \boldsymbol{H}_{o}^{*}\right\} d v}{\varepsilon_{o} \int_{V_{o}} \operatorname{Re}\left\{\boldsymbol{E} \cdot \boldsymbol{E}_{o}^{*}\right\} d v}=C_{o} \frac{(1+q)}{(1+p)}
$$

where

$$
\begin{gathered}
C_{o}=\frac{1}{2} \frac{\int_{V}\left(\varepsilon_{o} \varepsilon_{r e l} \boldsymbol{E}_{o} \cdot \boldsymbol{E}_{o}^{*}+\mu_{o} \boldsymbol{H}_{o} \cdot \boldsymbol{H}_{o}^{*}\right) d v}{\varepsilon_{o} \int_{V_{o}} \boldsymbol{E}_{o} \cdot \boldsymbol{E}_{o}^{*} d v} \\
p=\frac{\int_{V_{o}} \Re \mathrm{Re}\left\{\boldsymbol{e} \cdot \boldsymbol{E}_{o}^{*}\right\} d v}{\int_{V_{o}} \boldsymbol{E}_{o} \cdot \boldsymbol{E}_{o}^{*} d v}
\end{gathered}
$$

and

$$
q=\frac{\int_{V} \operatorname{Re}\left\{\varepsilon_{o} \varepsilon_{r e l} \boldsymbol{e} \cdot \boldsymbol{E}_{o}^{*}+\mu_{o} \boldsymbol{h} \cdot \boldsymbol{H}_{o}^{*}\right\} d v}{\int_{V}\left(\varepsilon_{o} \varepsilon_{r e l} \boldsymbol{E}_{o} \cdot \boldsymbol{E}_{o}^{*}+\mu_{o} \boldsymbol{H}_{o} \cdot \boldsymbol{H}_{o}^{*}\right) d v}
$$

In the above, the constant $C_{o}$ is a characteristic of the cavity and is independent of the sample. We recognize that $\left\langle W_{E}\right\rangle=(1 / 4) \varepsilon_{o} \int_{V} \varepsilon_{r e l} \boldsymbol{E}_{o} \cdot \boldsymbol{E}_{o}^{*} d v$ and $\left\langle W_{M}\right\rangle=(1 / 4) \mu_{o} \int_{V} \boldsymbol{H}_{o} \cdot \boldsymbol{H}_{o}^{*} d v$ are real quantities (assuming negligible loss in the sample tube i.e. $\epsilon_{t}$ is real) and respectively correspond to time average electrical and magnetic energies stored in the cavity. Since at resonance $\left\langle W_{E}\right\rangle=\left\langle W_{M}\right\rangle$, we can write $\int_{V}\left(\varepsilon_{o} \varepsilon_{r e l} \boldsymbol{E}_{o} \cdot \boldsymbol{E}_{o}^{*}+\mu_{o} \boldsymbol{H}_{o} \cdot \boldsymbol{H}_{o}^{*}\right) d v=8\left\langle W_{E}\right\rangle$. Similarly, the quantity $\left\langle W_{E o}\right\rangle=(1 / 4) \varepsilon_{o} \int_{V_{o}} \boldsymbol{E}_{o} \cdot \boldsymbol{E}_{o}^{*} d v$ corresponds to the time average electrical energy stored in the empty sample tube. Then the constant $C_{o}=\left\langle W_{E}\right\rangle /\left\langle W_{E o}\right\rangle$ is the ratio of the electric energy stored inside the sample tube volume to the electric energy stored in the cavity.

Summarizing the above, we have the relation $C=\chi C_{o}$. Now we can write $\Delta \varepsilon_{r}$ as: 


$$
\Delta \varepsilon=2 \chi C_{o} \frac{\Delta \omega}{\omega}
$$

In eq(B11), $\chi=(1+q) /(1+p)$ is an 'energy storage factor' that reflects the ratio of the electric energy stored inside the sample tube volume with the sample present to the total energy stored in the cavity relative to the same ratio when the tube is empty.

Having obtained the real part of the sample dielectric constant we now turn to the imaginary part. For this purpose we make use of eq(B7) and put it in a form that involves measured quantities. First we rewrite the surface integral in eq(B7) using the perturbation fields as:

$$
2 \int_{S} \Re \mathrm{e}\left\{\boldsymbol{E}_{o} \times \boldsymbol{H}_{o}^{*}\right\} \cdot d s+\int_{S} \Re \mathrm{R}\left\{\boldsymbol{E}_{o}^{*} \times \boldsymbol{h}+\boldsymbol{e} \times \boldsymbol{H}_{o}^{*}\right\} \cdot d \boldsymbol{s}+\omega \varepsilon_{o} \varepsilon_{s}^{\prime \prime} \int_{V_{o}} \Re \mathrm{e}\left\{\boldsymbol{E} \cdot \boldsymbol{E}_{o}^{*}\right\} d v=0
$$

Realizing from eq(B1) that the first term on the left disappears and using eq(B5) in the above, we recognize that

$$
\int_{V_{o}} \Re \mathrm{Re}\left\{\boldsymbol{E} \cdot \boldsymbol{E}_{o}^{*}\right\} d v \approx \int_{V_{o}} \Re \mathrm{Re}\left\{\boldsymbol{E} \cdot \boldsymbol{E}^{*}\right\} d v
$$

to the first order. Then using eq(B4) we can write

$$
\omega \varepsilon_{o} \varepsilon_{s}^{\prime \prime} \int_{V_{o}} \Re \mathrm{e}\left\{\boldsymbol{E} \cdot \boldsymbol{E}_{o}^{*}\right\} d v=-\left\{\int_{A} \Re \mathrm{e}\left\{\boldsymbol{E} \times \boldsymbol{H}^{*}\right\} \cdot d s-\int_{A} \Re \mathrm{e}\left\{\boldsymbol{E}_{o} \times \boldsymbol{H}_{o}^{*}\right\} \cdot d s\right\}
$$

Note that we can express the integrals on the right hand side of the above in terms of the Qfactor. The Q-factor is defined as:

$$
Q=\omega \frac{\text { Time AverageEnergy Stored }}{\text { Time Average Energy Loss / second }}
$$

Using this definition, we can write the second term on the right hand side of eq(B12) with the help of eq(B2) as:

$$
\begin{aligned}
-\frac{1}{2} \int_{A} \Re \mathrm{Re}\left\{\boldsymbol{E}_{o} \times \boldsymbol{H}_{o}^{*}\right\} \cdot d s & =\frac{\omega_{o}}{4 Q_{o}} \int_{V}\left(\varepsilon_{o} \varepsilon_{r e l} \boldsymbol{E}_{o} \cdot \boldsymbol{E}_{o}^{*}+\mu_{o} \boldsymbol{H}_{o} \cdot \boldsymbol{H}_{o}^{*}\right) d v \\
& =2 \frac{\omega_{o}}{Q_{o}}\left\langle W_{E}\right\rangle
\end{aligned}
$$

Similarly with the help of eq(B3), the first term on the right hand side of eq(B12) can be written as:

$$
-\frac{1}{2} \int_{A} \Re \mathrm{e}\left\{\boldsymbol{E} \times \boldsymbol{H}^{*}\right\} \cdot d s=\frac{\omega}{4 Q} \int_{V}\left(\varepsilon_{o} \Re \mathrm{Re}\left\{\tilde{\varepsilon}_{r e l}\right\} \boldsymbol{E} \cdot \boldsymbol{E}^{*}+\mu_{o} \boldsymbol{H} \cdot \boldsymbol{H}^{*}\right) d v
$$


Note that

$$
\int_{V}\left(\varepsilon_{o} \Re \mathrm{e}\left\{\widetilde{\boldsymbol{\varepsilon}}_{r e l}\right\} \boldsymbol{E} \cdot \boldsymbol{E}^{*}+\mu_{o} \boldsymbol{H} \cdot \boldsymbol{H}^{*}\right) d v=\int_{V}\left(\varepsilon_{o} \varepsilon_{r e l} \boldsymbol{E} \cdot \boldsymbol{E}^{*}+\mu_{o} \boldsymbol{H} \cdot \boldsymbol{H}^{*}\right) d v+\varepsilon_{o} \Delta \varepsilon \int_{V_{o}} \boldsymbol{E} \cdot \boldsymbol{E}^{*} d v
$$

Using the perturbation fields and eq(B9) and eq(B10), to the first order, the integrals on the right hand side of this expression can be written as:

$$
\int_{V_{o}} \boldsymbol{E} \cdot \boldsymbol{E}^{*} d v \approx(1+2 p) \int_{V_{o}} \boldsymbol{E}_{o} \cdot \boldsymbol{E}_{o}^{*} d v
$$

and

$$
\int_{V}\left(\varepsilon_{o} \varepsilon_{r e l} \boldsymbol{E} \cdot \boldsymbol{E}^{*}+\mu_{o} \boldsymbol{H} \cdot \boldsymbol{H}^{*}\right) d v \approx(1+2 q) \int_{V}\left(\varepsilon_{o} \varepsilon_{r e l} \boldsymbol{E}_{o} \cdot \boldsymbol{E}_{o}^{*}+\mu_{o} \boldsymbol{H}_{o} \cdot \boldsymbol{H}_{o}^{*}\right) d v
$$

Using these results along with eq(B11), we can write eq(B14) as:

$$
-\frac{1}{2} \int_{\boldsymbol{A}} \Re \mathrm{R}\left\{\boldsymbol{E} \times \boldsymbol{H}^{*}\right\} \cdot d s=2 \frac{\omega}{Q}\left\langle W_{E}\right\rangle\left(1+2 q+(1+2 p) \chi \frac{\Delta \omega}{\omega}\right)
$$

Since $\Delta \omega / \omega<10^{-4}$, we can ignore the third term in the brackets on the right hand side:

$$
-\frac{1}{2} \int_{A} \Re\left(\boldsymbol{E} \times \boldsymbol{H}^{*}\right\} \cdot d s \approx 2 \frac{\omega}{Q}\left\langle W_{E}\right\rangle(1+2 q)
$$

Using eq(B13) and eq(B15) in eq(B12) we obtain:

$$
\varepsilon_{o} \varepsilon_{s}^{\prime \prime} \int_{V_{o}} \Re \mathrm{e}\left\{\boldsymbol{E} \cdot \boldsymbol{E}_{o}^{*}\right\} d v \approx 4\left[\left(\frac{1}{Q}-\frac{1}{Q_{o}}\right)+\frac{2 q}{Q}\right]\left\langle W_{E}\right\rangle .
$$

In the above we have once again neglected a term of order $\Delta \omega / \omega$. Recognizing that $\varepsilon_{o} \int_{V_{o}} \boldsymbol{E} \cdot \boldsymbol{E}_{o}^{*} d v=4(1+p)\left\langle W_{E o}\right\rangle$, we obtain:

$$
\varepsilon_{s}^{\prime \prime} \approx \frac{C_{o}}{(1+p)}\left[\left(\frac{1}{Q}-\frac{1}{Q_{o}}\right)+\frac{2 q}{Q}\right]
$$

If we add and subtract $q / Q_{o}$ to the right hand side, we finally obtain

$$
\varepsilon_{s}^{\prime \prime} \approx \chi C_{o}\left[\left(\frac{1}{Q}-\frac{1}{Q_{o}}\right)+\frac{q}{(1+q)}\left(\frac{1}{Q}+\frac{1}{Q_{o}}\right)\right]
$$


This equation is more accurate than the commonly recognized form which can be obtained by ignoring the second term in $\mathrm{eq}(\mathrm{B} 16)$ as:

$$
\varepsilon_{s}^{\prime \prime} \approx \chi C_{o} \Delta\left(\frac{1}{Q}\right)
$$

where $\Delta(1 / Q)=\left(1 / Q-1 / Q_{o}\right)$.

\section{ERROR ANALYSIS}

The constant $C_{o}$ is very sensitive to the inner volume of the sample tube and is thus hard to predict accurately. However by using a reference liquid with known dielectric constant $\varepsilon_{r}=\varepsilon_{r}^{\prime}+i \varepsilon_{r}^{\prime \prime}$, the constant $C$ may be eliminated as follows:

$$
\frac{\varepsilon_{s}^{\prime}-1}{\varepsilon_{r}^{\prime}-1}=\frac{\chi}{\chi_{r}} \frac{\omega_{r}}{\omega} \frac{\Delta \omega}{\Delta \omega_{r}}
$$

where $\chi_{r}=\left(1+q_{r}\right) /\left(1+p_{r}\right)$ and $p_{r}$ and $q_{r}$ are obtained from eq(B3) and eq(B4) respectively when the reference liquid is used, and $\Delta \omega_{r}$ is the frequency shift corresponding to the reference liquid. Similarly assuming we can suffice with the approximate expression given by eq(B17), we can write for $\varepsilon_{s}^{\prime \prime}$ :

$$
\frac{\varepsilon_{s}^{\prime \prime}}{\varepsilon_{r}^{\prime \prime}}=\frac{\chi}{\chi_{r}} \frac{\Delta(1 / Q)}{\Delta\left(1 / Q_{r}\right)}
$$

Where $\Delta\left(1 / Q_{r}\right)=\left(1 / Q_{r}-1 / Q_{o}\right)$ and $Q_{r}$ is the Q-factor of the cavity when the reference liquid is introduced. In practice, instead of estimating $\chi$ and $\chi_{r}$, the approximations

$$
\frac{\varepsilon_{s}^{\prime}-1}{\varepsilon_{r}^{\prime}-1} \approx \frac{\omega_{r}}{\omega} \frac{\Delta \omega}{\Delta \omega_{r}}
$$

and

$$
\frac{\varepsilon_{s}^{\prime \prime}}{\varepsilon_{r}^{\prime \prime}} \approx \frac{\Delta(1 / Q)}{\Delta\left(1 / Q_{r}\right)}
$$

are used. This can be justified on the basis of the fact that $|p| \ll<1,|q| \ll<1,\left|p_{r}\right| \ll<1$ and $\left|q_{r}\right| \ll<1$. The above approximation yields better results if the dielectric constant of the reference liquid is close to that of the sample liquid. The dimensionless quantity $\left|\chi / \chi_{r}-1\right|$ is a direct measure of the fractional error in sample dielectric permittivity incurred in the perturbation approximations of eq(B20) and eq(B21). In the following we estimate this error and present its dependence on the sample tube diameter. 
In order to estimate the error we have to determine the constants $p, q, p_{r}$ and $q_{r}$. For this purpose we begin with the expression of the fields inside the cavity given as:

$$
\boldsymbol{E}= \begin{cases}A_{o 1} J_{0}\left(k \sqrt{\varepsilon_{r e l}} \rho\right) \hat{z} & 0 \leq \rho<a_{1} \\ {\left[A_{o 2} J_{0}\left(k \sqrt{\varepsilon_{\imath}} \rho\right)+B_{o 2} N_{0}\left(k \sqrt{\varepsilon_{t}} \rho\right)\right] \hat{z}} & a_{1}<\rho<a_{2} \\ {\left[A_{o 3} J_{0}(k \rho)+B_{o 3} N_{0}(k \rho)\right] \hat{z}} & a_{2}<\rho<a_{3}\end{cases}
$$

and

$$
\boldsymbol{H}=\left\{\begin{array}{lr}
\eta_{o}^{-1} \eta_{r e l}^{-1} A_{o 1} J_{1}\left(k \sqrt{\varepsilon_{r e l}} \rho\right) \hat{\phi} & 0 \leq \rho<a_{1} \\
\eta_{t}^{-1}\left[A_{o 2} J_{1}\left(k \sqrt{\varepsilon_{t}} \rho\right)+B_{o 2} N_{1}\left(k \sqrt{\varepsilon_{i}} \rho\right)\right] \hat{\phi} & a_{1}<\rho<a_{2} \\
\eta_{o}^{-1}\left[A_{o 3} J_{1}(k \rho)+B_{o 3} N_{1}(k \rho)\right] \hat{\phi} & a_{2}<\rho<a_{3}
\end{array}\right.
$$

where $\eta_{r e l}=1 / \sqrt{\varepsilon_{r e l}}$. For the empty cavity one has $k=k_{o}=\omega_{o} \sqrt{\varepsilon_{o} \mu_{o}}$ and $\varepsilon_{\text {rel }}=1$. Similar expressions are valid when the sample liquid is inserted into the sample tube. In this case, $k=k_{o}+\Delta \omega /\left(c \sqrt{\varepsilon_{s}}\right), \varepsilon_{r e l}=\varepsilon_{s}$ and the coefficients $A_{o i}$ and $B_{o i}$ are replaced by $A_{s i}$ and $B_{s i}$ respectively for $i=\{1,2,3\}$. In the case of the reference liquid, $k=k_{o}+\Delta \omega_{r} /\left(c \sqrt{\varepsilon_{r}}\right), \varepsilon_{r e l}=\varepsilon_{r}$, and the coefficients $A_{o i}$ and $B_{o i}$ are replaced by $A_{r i}$ and $B_{r i}$ respectively for $i=\{1,2,3\}$. The coefficients are found by applying the boundary conditions i.e. the continuity of $\boldsymbol{E}$ and $\boldsymbol{H}$ fields at $\rho=a_{1}, \rho=a_{2}$ and $\rho=a_{3}$ (note that both field have only tangential components to the boundaries). It is assumed that the walls and end plates are perfectly conducting. This leads to the following relations

$$
\left[\begin{array}{c}
A_{o i} \\
B_{o i}
\end{array}\right]=\left[\Gamma_{o i}\right]\left[\begin{array}{l}
A_{o 1} \\
B_{o 1}
\end{array}\right], \quad\left[\begin{array}{l}
A_{s i} \\
B_{s i}
\end{array}\right]=\left[\Gamma_{s i}\right]\left[\begin{array}{c}
A_{s 1} \\
B_{s 1}
\end{array}\right] \quad \text { and } \quad\left[\begin{array}{c}
A_{r i} \\
B_{r i}
\end{array}\right]=\left[\Gamma_{r i}\right]\left[\begin{array}{c}
A_{r 1} \\
B_{r 1}
\end{array}\right]
$$

where $\left[\Gamma_{o i}\right],\left[\Gamma_{s i}\right]$ and $\left[\Gamma_{r i}\right]$ are the transition matrices for the empty cavity, cavity with sample liquid and cavity with reference liquid respectively, relating the coefficients of the $i$ 'th layer to those of the first layer. The generic transition matrix is given by

$$
\left[\Gamma_{i}\right]=\prod_{p=1}^{i-1}\left[T_{p}\right]
$$

where 


$$
\begin{aligned}
& \frac{2}{\pi X_{p+1, p}}\left[T_{p}\right] \\
& =\left[\begin{array}{ll}
n_{p} N_{0}\left(X_{p+1, p}\right) J_{1}\left(X_{p, p}\right)-N_{1}\left(X_{p+1, p}\right) J_{0}\left(X_{p, p}\right) & n_{p} N_{0}\left(X_{p+1, p}\right) N_{1}\left(X_{p, p}\right)-N_{1}\left(X_{p+1, p}\right) N_{0}\left(X_{p, p}\right) \\
-n_{p} J_{0}\left(X_{p+1, p}\right) J_{1}\left(X_{p, p}\right)+J_{1}\left(X_{p+1, p}\right) J_{0}\left(X_{p, p}\right) & -n_{p} J_{0}\left(X_{p+1, p}\right) N_{1}\left(X_{p, p}\right)+J_{1}\left(X_{p+1, p}\right) N_{0}\left(X_{p, p}\right)
\end{array}\right]
\end{aligned}
$$

with $X_{p, q}=k \sqrt{\varepsilon_{p}} a_{q}, n_{p}=\sqrt{\varepsilon_{p} / \varepsilon_{p+1}}$ and $\varepsilon_{p}$ is the relative dielectric permittivity of the $p$ 'th layer.

Using the field expressions, we can now obtain explicit expressions for the constants $p, q, p_{r}$ and $q_{r}$. However, in order to obtain simple expressions we make several approximations. Since $a_{1}$ is so small that $k \sqrt{\varepsilon_{r e l}} a_{1} \ll 1$, we can write $J_{0}\left(\left\{k_{o}+\Delta \omega /\left(c \sqrt{\varepsilon_{s}}\right)\right\} \sqrt{\varepsilon_{s}} \rho\right) \approx J_{0}\left(k_{o} \rho\right) \approx 1$. Hence, in the region $0 \leq \rho<a_{1}$ we have $e=A_{s 1} J_{0}\left(k_{s} \rho\right) \hat{z}-A_{o 1} J_{0}\left(k_{o} \rho\right) \hat{z} \approx\left(A_{s 1}-A_{o 1}\right) \hat{z}$. Thus from eq(B9)

$$
p \approx \frac{\varepsilon_{o}\left(\Re \mathrm{e}\left\{A_{s 1}\right\} / A_{o 1}-1\right) V_{1}}{\varepsilon_{o} V_{1}}=\frac{\Re \mathrm{e}\left\{A_{s 1}\right\}}{A_{o 1}}-1
$$

and consequently $1+p \approx \operatorname{Re}\left\{A_{s 1}\right\} / A_{o 1}$ for the sample liquid. Similarly, for the reference liquid we obtain $1+p_{r} \approx \Re \in \mathcal{R}\left\{A_{r 1}\right\} / A_{o 1}$. For the quantity $q$ given in eq(B10), we first note that $a_{1}, a_{2} \ll a_{3}$, hence eq(B10) can be approximated by

$$
q \approx \frac{\int_{V_{3}} \Re \mathrm{e}\left\{\varepsilon_{o} \varepsilon_{r e l} \boldsymbol{e} \cdot \boldsymbol{E}_{o}^{*}+\mu_{o} \boldsymbol{h} \cdot \boldsymbol{H}_{o}^{*}\right\} d v}{\int_{V_{3}}\left(\varepsilon_{o} \varepsilon_{r e l} \boldsymbol{E}_{o} \cdot \boldsymbol{E}_{o}^{*}+\mu_{o} \boldsymbol{H}_{o} \cdot \boldsymbol{H}_{o}^{*}\right) d v}
$$

Since $\Delta \omega a_{3} / c \ll 1$, we can write $J_{n}\left(\left[k_{o}+\Delta \omega / c\right] \rho\right) \approx J_{n}\left(k_{o} \rho\right)$ in the region $a_{2}<\rho<a_{3}$. This approximation leads to $\boldsymbol{e} \approx\left(A_{s 3}-A_{o 3}\right) J_{0}\left(k_{o} \rho\right) \hat{z}$ and $\boldsymbol{h} \approx \eta_{o}^{-1}\left(A_{s 3}-A_{o 3}\right) N_{1}\left(k_{o} \rho\right) \hat{\phi}$ in the pertinent region. Thus

$$
q \approx \frac{\left(\Re \operatorname{Re}\left\{A_{s 3}\right\}-A_{o 3}\right) I}{A_{o 3} I}=\operatorname{Re}\left\{A_{s 3}\right\} / A_{o 3}-1
$$

where $I=\int_{a_{2}}^{a_{3}} \rho\left(\varepsilon_{o} J_{0}^{2}\left(k_{o} \rho\right)+\eta_{o}^{-1} \mu_{o} J_{1}^{2}\left(k_{o} \rho\right)\right) d \rho$. With this final expression we obtain the result $1+q \approx \operatorname{Re}\left\{A_{s 3}\right\} / A_{o 3}$. Note that $A_{o 3} / A_{o 1}=\left[\Gamma_{o 3}\right]_{11} \dot{=} \gamma_{o}$ (real quantity) and $A_{s 3} / A_{s 1}=\left[\Gamma_{s 3}\right]_{11} \dot{\dot{ }}=\gamma_{s}$. Hence with the help these relations, we finally obtain $\chi \approx\left(\Re \mathrm{Re}\left\{A_{s 3}\right\} / \Re \mathrm{e}\left\{A_{s 1}\right\}\right)\left(A_{o 1} / A_{o 3}\right)$ $\approx \Re \mathrm{Re}\left\{A_{s 3} / A_{s 1}\right\}\left(A_{o 1} / A_{o 3}\right)=\operatorname{Re}\left\{\gamma_{s}\right\} / \gamma_{o}$. In this final expression, the second approximation is based on the premise that in $V_{3}$ the fields are negligibly perturbed when the sample is introduced so 
that these fields are almost real. Similarly, defining $\gamma_{r} \dot{=} A_{r 3} / A_{r 1}=\left[\Gamma_{r 3}\right]_{11}$ for the reference liquid we obtain $\chi_{r} \approx \operatorname{Re}\left\{\gamma_{r}\right\} / \gamma_{o}$. With this final, relation the fractional error reduces to $\left|\chi / \chi_{r}-1\right| \approx\left|\Re \mathrm{e}\left\{\gamma_{s}\right\} / \Re \mathrm{e}\left\{\gamma_{r}\right\}-1\right|$. 


\section{Appendix C Measurement Data}

This appendix contains three subsections. In the first subsection, the network configuration (shown in Figure 7) is given for the methanol and seawater measurements in four separate tables. In subsection C.2 the seawater measurement data is given is three tables. Finally, in subsection C.3, a table of the frequency drift over the time span of an experiment is given for several representative cases.

\section{C.1 Configuration Data}

\begin{tabular}{|c|c|c|c|c|c|c|c|}
\hline \multicolumn{7}{|c|}{ Methanol Measurement Configuration } \\
\hline Date & Exp\# & Methanol & Tube & Temp & Isolator & Attenuator & Amplifier \\
\hline $01 / 26 / 07$ & 1 & New & $\# 12$ & $20^{\circ} \mathrm{C}$ & Yes & No & Yes \\
\hline $01 / 26 / 07$ & 2 & New & $\# 12$ & $20^{\circ} \mathrm{C}$ & Yes & No & Yes \\
\hline $01 / 26 / 07$ & 3 & Old & $\# 12$ & $20^{\circ} \mathrm{C}$ & Yes & No & Yes \\
\hline $01 / 27 / 07$ & 1 & New & $\# 12$ & $20^{\circ} \mathrm{C}$ & Yes & No & Yes \\
\hline $02 / 01 / 08$ & 1 & New & $\# 12$ & $20^{\circ} \mathrm{C}$ & Yes & No & Yes \\
\hline $02 / 04 / 08$ & 2 & New & $\# 12$ & $20^{\circ} \mathrm{C}$ & Yes & No & Yes \\
\hline $04 / 10 / 08$ & 1 & New & $\# 12$ & $20^{\circ} \mathrm{C}$ & Yes & Yes & No \\
\hline $04 / 19 / 08$ & 1 & New & $\# 12$ & $20^{\circ} \mathrm{C}$ & Yes & Yes & No \\
\hline $04 / 24 / 08$ & 1 & New & $\# 12$ & $20^{\circ} \mathrm{C}$ & Yes & Yes & No \\
\hline $04 / 24 / 08$ & 2 & New & $\# 12$ & $20^{\circ} \mathrm{C}$ & Yes & Yes & No \\
\hline $04 / 25 / 08$ & 1 & New & $\# 12$ & $20^{\circ} \mathrm{C}$ & Yes & Yes & No \\
\hline $04 / 29 / 08$ & 1 & New & $\# 12$ & $20^{\circ} \mathrm{C}$ & Yes & Yes & No \\
\hline $04 / 30 / 08$ & 1 & New & $\# 12$ & $20^{\circ} \mathrm{C}$ & Yes & Yes & No \\
\hline $04 / 30 / 08$ & 2 & New & $\# 12$ & $20^{\circ} \mathrm{C}$ & Yes & Yes & No \\
\hline $05 / 13 / 08$ & 1 & New & $\# 12$ & $20^{\circ} \mathrm{C}$ & Yes & Yes & No \\
\hline
\end{tabular}

Table C.1-1. Configuration for Methanol Measurements 


\begin{tabular}{|c|c|c|c|c|c|c|c|}
\hline \multicolumn{7}{|c|}{ Seawater Measurement Configuration (30.002 psu) } \\
\hline Date & Tube & Temp & Exp \# & Isolators & Attenuators & Amplifier & Freq Stabilizer \\
\hline $12 / 14 / 07$ & $\# 12$ & 10 & 1 & Yes & No & Yes & Yes \\
\hline $12 / 28 / 07$ & $\# 12$ & 10 & 1 & Yes & No & Yes & Yes \\
\hline $12 / 28 / 07$ & $\# 12$ & 10 & 2 & Yes & No & Yes & Yes \\
\hline $02 / 08 / 08$ & $\# 12$ & 10 & 1 & Yes & No & Yes & Yes \\
\hline $12 / 29 / 07$ & $\# 12$ & 15 & 1 & Yes & No & Yes & Yes \\
\hline $12 / 29 / 07$ & $\# 12$ & 15 & 2 & Yes & No & Yes & Yes \\
\hline $02 / 07 / 08$ & $\# 12$ & 15 & 1 & Yes & No & Yes & Yes \\
\hline $12 / 09 / 07$ & $\# 12$ & 20 & 1 & Yes & No & Yes & Yes \\
\hline $12 / 12 / 07$ & $\# 12$ & 20 & 1 & Yes & No & Yes & Yes \\
\hline $12 / 29 / 07$ & $\# 12$ & 20 & 1 & Yes & No & Yes & Yes \\
\hline $12 / 30 / 07$ & $\# 12$ & 20 & 1 & Yes & No & Yes & Yes \\
\hline $12 / 30 / 07$ & $\# 12$ & 20 & 2 & Yes & No & Yes & Yes \\
\hline $02 / 07 / 08$ & $\# 12$ & 20 & 1 & Yes & No & Yes & Yes \\
\hline $12 / 26 / 07$ & $\# 12$ & 25 & 1 & Yes & No & Yes & Yes \\
\hline $12 / 28 / 07$ & $\# 12$ & 25 & 1 & Yes & No & Yes & Yes \\
\hline $12 / 30 / 07$ & $\# 12$ & 25 & 1 & Yes & No & Yes & Yes \\
\hline $02 / 05 / 08$ & $\# 12$ & 25 & 1 & Yes & No & Yes & Yes \\
\hline $02 / 05 / 08$ & $\# 12$ & 25 & 2 & Yes & No & Yes & Yes \\
\hline $02 / 06 / 08$ & $\# 12$ & 25 & 1 & Yes & No & Yes & Yes \\
\hline $12 / 26 / 07$ & $\# 12$ & 30 & 1 & Yes & No & Yes & Yes \\
\hline $12 / 27 / 07$ & $\# 12$ & 30 & 1 & Yes & No & Yes & Yes \\
\hline $02 / 10 / 08$ & $\# 12$ & 30 & 1 & Yes & No & Yes & Yes \\
\hline $12 / 15 / 07$ & $\# 12$ & 35 & 1 & Yes & No & Yes & Yes \\
\hline $12 / 27 / 07$ & $\# 12$ & 35 & 1 & Yes & No & Yes & Yes \\
\hline $12 / 27 / 07$ & $\# 12$ & 35 & 2 & Yes & No & Yes & Yes \\
\hline $02 / 10 / 08$ & $\# 12$ & 35 & 1 & Yes & No & Yes & Yes \\
\hline
\end{tabular}

Table C.1-2. Network Configuration for Seawater Measurements with Salinity 30.002 psu

\begin{tabular}{|c|c|c|c|c|c|c|c|}
\hline \multicolumn{7}{|c|}{ Seawater Measurement Configuration (34.997 psu) } \\
\hline Date & Tube & Temp & Exp \# & Isolators & Attenuators & Amplifier & Freq Stabilizer \\
\hline $05 / 20 / 08$ & $\# 12$ & 10 & 1 & Yes & Yes & No & Yes \\
\hline $05 / 30 / 08$ & $\# 12$ & 10 & 1 & Yes & Yes & No & Yes \\
\hline $05 / 20 / 08$ & $\# 12$ & 15 & 1 & Yes & Yes & No & Yes \\
\hline $05 / 30 / 08$ & $\# 12$ & 15 & 1 & Yes & Yes & No & Yes \\
\hline $05 / 19 / 08$ & $\# 12$ & 20 & 1 & Yes & Yes & No & Yes \\
\hline $05 / 29 / 08$ & $\# 12$ & 20 & 1 & Yes & Yes & No & Yes \\
\hline $05 / 19 / 08$ & $\# 12$ & 25 & 1 & Yes & Yes & No & Yes \\
\hline $05 / 28 / 09$ & $\# 12$ & 25 & 1 & Yes & Yes & No & Yes \\
\hline $05 / 27 / 09$ & $\# 12$ & 30 & 1 & Yes & Yes & No & Yes \\
\hline $05 / 28 / 08$ & $\# 12$ & 30 & 1 & Yes & Yes & No & Yes \\
\hline $06 / 02 / 08$ & $\# 12$ & 30 & 1 & Yes & Yes & No & Yes \\
\hline $06 / 04 / 08$ & $\# 12$ & 30 & 1 & Yes & Yes & No & Yes \\
\hline $05 / 27 / 08$ & $\# 12$ & 35 & 1 & Yes & Yes & No & Yes \\
\hline $05 / 29 / 08$ & $\# 12$ & 35 & 1 & Yes & Yes & No & Yes \\
\hline
\end{tabular}

Table C.1-3. Network Configuration for Seawater Measurements with Salinity 34.997 psu 


\begin{tabular}{|c|c|c|c|c|c|c|c|}
\hline \multicolumn{7}{|c|}{ Seawater Measurement Configuration (38.274 psu) } \\
\hline Date & Tube & Temp & Exp \# & Isolators & Attenuators & Amplifier & Freq Stabilizer \\
\hline $03 / 05 / 08$ & $\# 12$ & 10 & 1 & Yes & No & No & Yes \\
\hline $05 / 02 / 08$ & $\# 12$ & 10 & 1 & Yes & Yes & No & Yes \\
\hline $03 / 04 / 08$ & $\# 12$ & 15 & 1 & Yes & No & No & Yes \\
\hline $03 / 05 / 08$ & $\# 12$ & 15 & 1 & Yes & No & No & Yes \\
\hline $05 / 02 / 08$ & $\# 12$ & 15 & 1 & Yes & Yes & No & Yes \\
\hline $03 / 05 / 08$ & $\# 12$ & 20 & 1 & Yes & No & No & Yes \\
\hline $05 / 01 / 08$ & $\# 12$ & 20 & 1 & Yes & Yes & No & Yes \\
\hline $05 / 08 / 08$ & $\# 12$ & 20 & 1 & Yes & Yes & No & Yes \\
\hline $03 / 03 / 08$ & $\# 12$ & 25 & 1 & Yes & No & No & Yes \\
\hline $05 / 01 / 08$ & $\# 12$ & 25 & 1 & Yes & Yes & No & Yes \\
\hline $03 / 03 / 08$ & $\# 12$ & 30 & 1 & Yes & No & No & Yes \\
\hline $05 / 05 / 08$ & $\# 12$ & 30 & 1 & Yes & Yes & No & Yes \\
\hline $05 / 06 / 08$ & $\# 12$ & 30 & 1 & Yes & Yes & No & Yes \\
\hline $05 / 14 / 09$ & $\# 12$ & 30 & 1 & Yes & Yes & No & Yes \\
\hline $05 / 23 / 08$ & $\# 12$ & 30 & 1 & Yes & Yes & No & Yes \\
\hline $03 / 04 / 08$ & $\# 12$ & 35 & 1 & Yes & No & No & Yes \\
\hline $05 / 05 / 08$ & $\# 12$ & 35 & 1 & Yes & Yes & No & Yes \\
\hline $05 / 06 / 08$ & $\# 12$ & 35 & 1 & Yes & Yes & No & Yes \\
\hline $05 / 15 / 08$ & $\# 12$ & 35 & 1 & Yes & Yes & No & Yes \\
\hline $05 / 23 / 08$ & $\# 12$ & 35 & 1 & Yes & Yes & No & Yes \\
\hline
\end{tabular}

Table C.1-4. Network Configuration for Seawater Measurements with Salinity 38.274 psu 


\section{C.2 Seawater Dielectric Constant Data}

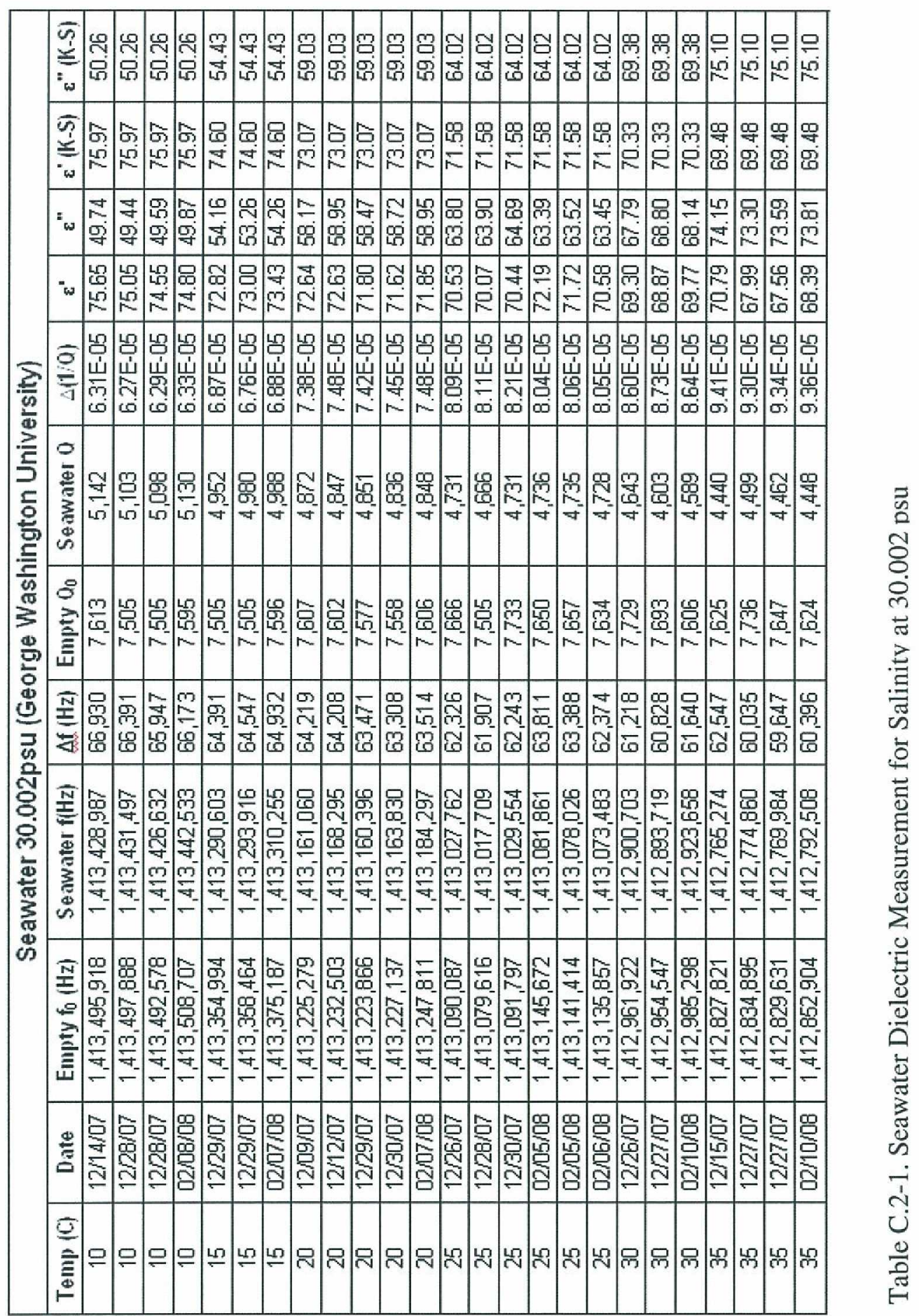



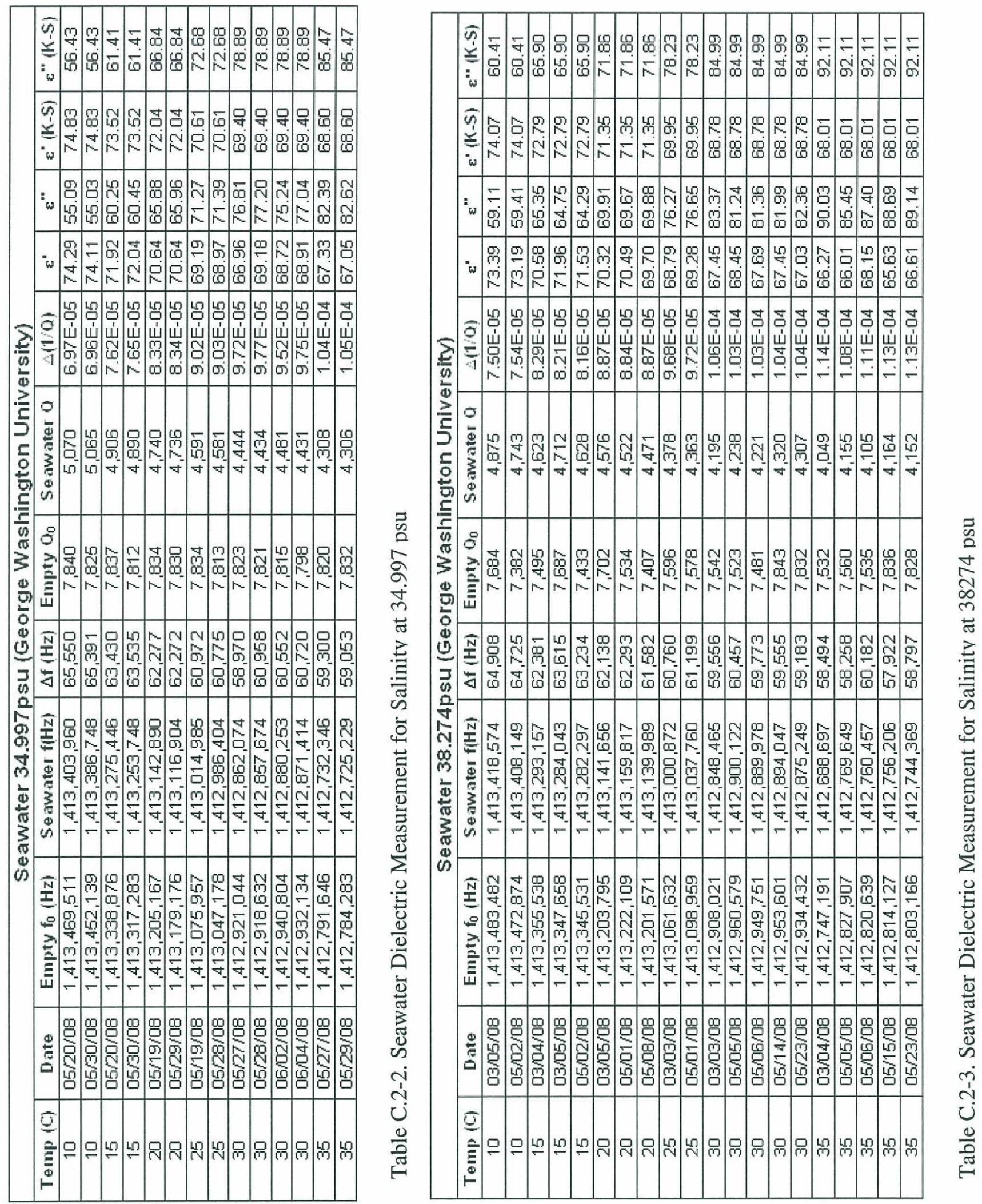


\section{C.3 Frequency Stability}

In this subsection of Appendix C, the long term frequency stability of the system is discussed. To help with the discussion, a frequency stability table has been constructed. In this table the difference between the start resonant frequency and the stop resonant frequency is recorded for 18 different experiments. The time difference is also recorded. Here it is seen that the average frequency drift over one and a half hours is approximately $1 \mathrm{KHz}$. Since the cavity is held at a stable temperature $\left( \pm 0.02^{\circ} \mathrm{C}\right)$, it is believed the reasons for this drift are changes is the room temperature. There is, however, no observable correlation between changes in the room temperature and changes in the cavity resonant frequency. This is because the temperature changes in the room have an integrated effect on the network external to the liquid bath. Although these resonant frequency changes occur, they do not have a noticeable effect on the measurements since they occur slowly. They do however, make it necessary to do the measurements one at a time rather than automating the system. This has seriously slowed down the amount of data we could collect. One proposed solution has been to put the isolators and attenuators in the liquid bath. Their temperatures would be held constant and thus the effects of external temperature changes would be much reduced.

\begin{tabular}{|c|c|c|c|c|c|c|c|c|c|}
\hline \multicolumn{10}{|c|}{ Frequency Stability (Seawater) } \\
\hline Date & psu & $\begin{array}{c}\text { Temp } \\
\left({ }^{\circ} \mathrm{C}\right)\end{array}$ & Att. & Start Freq.(Hz) & Start Time & End Freq.(Hz) & End Time & $\Delta f(H z)$ & $\Delta t$ \\
\hline $12 / 28 / 07$ & 30.002 & 10 & $\mathrm{~N}$ & $1,413,492,578$ & $10: 49$ & $1,413,491,691$ & $13: 03$ & 887 & $2: 13$ \\
\hline $12 / 29 / 07$ & 30.002 & 15 & $\mathrm{~N}$ & $1,413,492,578$ & $4: 24$ & $1,413,357,723$ & $7: 22$ & 2729 & $2: 58$ \\
\hline $02 / 07 / 08$ & 30.002 & 20 & $\mathrm{~N}$ & $1,413,492,578$ & $14: 45$ & $1,413,249,334$ & $18: 05$ & 1523 & $3: 19$ \\
\hline $12 / 30 / 07$ & 30.002 & 25 & $\mathrm{~N}$ & $1,413,492,578$ & $18: 48$ & $1,413,092,123$ & $20: 35$ & 326 & $1: 46$ \\
\hline $12 / 26 / 07$ & 30.002 & 30 & $\mathrm{~N}$ & $1,413,492,578$ & $9: 38$ & $1,412,961,884$ & $12: 25$ & 38 & $2: 47$ \\
\hline $12 / 27 / 07$ & 30.002 & 35 & $\mathrm{~N}$ & $1,413,492,578$ & $4: 25$ & $1,412,832,612$ & $6: 55$ & 2283 & $2: 29$ \\
\hline $05 / 30 / 08$ & 34.997 & 10 & $\mathrm{Y}$ & $1,413,492,578$ & $16: 44$ & $1,413,450,930$ & $18: 55$ & 1209 & $2: 11$ \\
\hline $05 / 20 / 08$ & 34.997 & 15 & $\mathrm{Y}$ & $1,413,492,578$ & $10: 58$ & $1,413,339,361$ & $12: 33$ & 485 & $1: 35$ \\
\hline $05 / 29 / 08$ & 34.997 & 20 & $\mathrm{Y}$ & $1,413,492,578$ & $12: 57$ & $1,412,781,762$ & $14: 36$ & 2521 & $1: 38$ \\
\hline $05 / 19 / 08$ & 34.997 & 25 & $\mathrm{Y}$ & $1,413,492,578$ & $11: 49$ & $1,413,076,114$ & $12: 57$ & 157 & $1: 07$ \\
\hline $05 / 27 / 08$ & 34.997 & 30 & $\mathrm{Y}$ & $1,413,492,578$ & $12: 57$ & $1,412,920,989$ & $14: 10$ & 55 & $1: 13$ \\
\hline $05 / 27 / 08$ & 34.997 & 35 & $\mathrm{Y}$ & $1,413,492,578$ & $15: 09$ & $1,412,789,412$ & $16: 36$ & 2234 & $1: 27$ \\
\hline $05 / 02 / 08$ & 38.274 & 10 & $\mathrm{Y}$ & $1,413,492,578$ & $16: 57$ & $1,413,473,408$ & $18: 44$ & 534 & $1: 46$ \\
\hline $03 / 04 / 08$ & 38.274 & 15 & $\mathrm{~N}$ & $1,413,492,578$ & $10: 56$ & $1,413,357,029$ & $12: 05$ & 1491 & $1: 09$ \\
\hline $05 / 01 / 08$ & 38.274 & 20 & $\mathrm{Y}$ & $1,413,492,578$ & $10: 52$ & $1,413,221,754$ & $12: 36$ & 355 & $1: 43$ \\
\hline $05 / 01 / 08$ & 38.274 & 25 & $\mathrm{Y}$ & $1,413,492,578$ & $15: 05$ & $1,413,098,226$ & $16: 35$ & 733 & $1: 30$ \\
\hline $05 / 06 / 08$ & 38.274 & 30 & $\mathrm{Y}$ & $1,413,492,578$ & $11: 20$ & $1,412,949,791$ & $12: 56$ & 40 & $1: 35$ \\
\hline $05 / 06 / 08$ & 38.274 & 35 & $\mathrm{Y}$ & $1,413,492,578$ & $14: 49$ & $1,412,818,111$ & $16: 31$ & 2528 & $1: 42$ \\
\hline Average & & & & & & & & 1118 & $1: 54$ \\
\hline $\begin{array}{l}\text { Standard } \\
\text { Deviation }\end{array}$ & & & & & & & & 970 & $0: 38$ \\
\hline
\end{tabular}

Table C.3-1 Frequency Stability Table. Start and end times occur on the same day. 


\section{Appendix D \\ Loaded and Unloaded Q}

In this appendix, the subject of loaded and unloaded $\mathrm{Q}$ will be discussed. The formulas for the imaginary dielectric constant given in eq(2a) assume that the Q used is the unloaded $Q$. It will be denoted by $Q_{\mathrm{UL}}$. The Q measurement taken by the NA is the loaded Q that will be denoted by $Q_{\mathrm{L}}$. For transmission cavities the two are related by

$$
\mathrm{Q}_{\mathrm{UL}}=\mathrm{Q}_{\mathrm{L}}\left(1+\beta_{1}+\beta_{2}\right)
$$

where $\beta_{1}$ and $\beta_{2}$ are the input and output coupling coefficients. For a discussion of these quantities, please refer to the "Measurement of Q" by M. Sucher (1963) or Kajfez et al. (1999). If the cavity is viewed as a reciprocal two port, its scattering parameters are $S_{11}, S_{21}$ and $S_{22}$. The scattering parameter $S_{21}$ is directly related to the coupling coefficients. At the resonant frequency of the cavity, the relationship is given by

$$
S_{21}=\frac{2 \sqrt{\beta_{1} \beta_{2}}}{1+\beta_{1}+\beta_{2}}
$$

If it is assumed that the cavity coupling coefficients are the same (approximately true for our cavity) then $\beta=\beta_{1}=\beta_{2}$ and eq(D2) can be solved for $\beta$, thus

$$
2 \beta=\frac{S_{21}}{1-S_{21}}
$$

Equations (D1) and (D3) can be applied to our Q measurements to determine $Q_{\mathrm{UL}}$ from $Q_{\mathrm{L}}$. First, it is noted that the magnitude of $S_{21}$ is measured by the NA between terminals a and $b$ of Figure 7. This is not the $S_{21}$ of the cavity, but knowing the network configuration given in Appendix C.1, the cavity $S_{21}$ can be calculated. When only isolators are used, $1 \mathrm{~dB}$ is subtracted from the $\mathrm{S}_{21}$ computed by the NA while when both isolators and attenuators are used, $11 \mathrm{~dB}$ is subtracted.

Some results of these calculations are presented in Table D-1 for the empty cavity Q and for the Q when the cavity has a sample. The columns marked $\left(S_{21}^{E}\right)_{d B}$ and $\left(S_{21}^{S}\right)_{d B}$ are the cavity values of $\mathrm{S}_{21}$ in $\mathrm{dB}$ for the empty cavity and for the cavity with a sample in it respectively. An examination of the table shows that the loaded and unloaded Q's can differ by as much 300 to 500 . However, when $\Delta(1 / Q)$ is computed for the loaded and unloaded cases, there is very little difference as demonstrated by the \%Error column. The errors are under $0.1 \%$ in general and thus there is no need to compute the unloaded Q when calculating the imaginary part of the sample dielectric constant from eq(2a). 


\begin{tabular}{|c|c|c|c|c|c|c|c|c|c|c|c|c|c|}
\hline \multicolumn{14}{|c|}{ LOADED and UNLOADED $Q$} \\
\hline Date & psu/Meth. & Temp (C) & Att & $\left(\mathrm{L}_{\mathrm{NW}}\right)_{\mathrm{dB}}$ & $Q_{L}{ }^{E}$ & $\left(S_{21}{ }^{E}\right)_{d B}$ & $Q_{U L}{ }^{E}$ & $Q_{L}{ }^{\mathbf{S}}$ & $\left(S_{21}\right)_{d B}$ & $\mathbf{Q}_{\mathrm{UL}}{ }^{\mathrm{S}}$ & $\Delta\left(1 / Q_{L}\right)$ & $\Delta\left(1 / Q_{U L}\right)$ & $\%$ Error \\
\hline $02 / 08 / 08$ & 30.002 & 10 & $\mathrm{~N}$ & 1 & 7595 & -26.15 & 8039 & 5130 & -29.54 & 5329 & 6.327E-05 & $6.325 \mathrm{E}-05$ & $0.02 \%$ \\
\hline $12 / 29 / 07$ & 30.002 & 15 & $\mathrm{~N}$ & 1 & 7504 & -24.99 & 8010 & 4980 & -28.66 & 5195 & 6.754E-05 & $6.765 \mathrm{E}-05$ & $0.16 \%$ \\
\hline $12 / 30 / 07$ & 30.002 & 20 & $\mathrm{~N}$ & 1 & 7577 & -27.35 & 7960 & 4851 & -31.24 & 5005 & $7.416 \mathrm{E}-05$ & $7.417 \mathrm{E}-05$ & $0.01 \%$ \\
\hline $12 / 30 / 07$ & 30.002 & 25 & $\mathrm{~N}$ & 1 & 7733 & -29.65 & 8030 & 4731 & -33.84 & 4841 & 8.206E-05 & $8.201 \mathrm{E}-05$ & $0.05 \%$ \\
\hline $12 / 27 / 07$ & 30.002 & 30 & $\mathrm{~N}$ & 1 & 7693 & -28.51 & 8031 & 4603 & -33.03 & 4721 & 8.726E-05 & 8.729E-05 & $0.04 \%$ \\
\hline $12 / 28 / 07$ & 30.002 & 35 & $\mathrm{~N}$ & 1 & 7647 & -26.02 & 8101 & 4462 & -30.73 & 4613 & $9.334 \mathrm{E}-05$ & 9.337E-05 & $0.02 \%$ \\
\hline $05 / 20 / 08$ & 34.997 & 10 & $Y$ & 11 & 7840 & -30.14 & 8813 & 5070 & -33.94 & 5459 & 6.969E-05 & $6.972 \mathrm{E}-05$ & $0.05 \%$ \\
\hline $05 / 20 / 08$ & 34.997 & 15 & $\mathrm{Y}$ & 11 & 7837 & -30.33 & 8786 & 4906 & -34.41 & 5261 & 7.623E-05 & $7.625 \mathrm{E}-05$ & $0.02 \%$ \\
\hline $05 / 29 / 08$ & 34.997 & 20 & $\mathrm{Y}$ & 11 & 7830 & -31.29 & 8669 & 4736 & -35.63 & 5031 & 8.343E-05 & $8.340 \mathrm{E}-05$ & $0.04 \%$ \\
\hline 05/19/08 & 34.997 & 25 & $Y$ & 11 & 7834 & -31.16 & 8687 & 4591 & -35.80 & 4871 & $9.017 \mathrm{E}-05$ & $9.017 \mathrm{E}-05$ & $0.00 \%$ \\
\hline $05 / 28 / 08$ & 34.997 & 30 & $\mathrm{Y}$ & 11 & 7821 & -31.54 & 8632 & 4434 & -36.40 & 4686 & 9.767E-05 & E-05 & $0.10 \%$ \\
\hline $05 / 27 / 08$ & 34.997 & 35 & $Y$ & 11 & 7820 & \begin{tabular}{|l|}
-32.62 \\
\end{tabular} & 8528 & 4308 & -37.90 & 4512 & E-04 & $=-04$ & $0.12 \%$ \\
\hline $05 / 02 / 08$ & 38.274 & 10 & $Y$ & 11 & 7382 & -29.98 & 8317 & 4743 & -33.84 & 5112 & 7.537E-05 & $7.539 \mathrm{E}-05$ & $0.02 \%$ \\
\hline $05 / 02 / 08$ & 38.274 & 15 & $\mathrm{Y}$ & 11 & 7433 & -29.80 & 8397 & 4628 & -33.93 & 4984 & E-05 & E-05 & $0.02 \%$ \\
\hline $05 / 01 / 08$ & 38.274 & 20 & $\mathrm{Y}$ & 11 & 7534 & -29.80 & 8512 & 4522 & -34.28 & 4855 & E-05 & E-05 & $0.10 \%$ \\
\hline $03 / 05 / 08$ & 38.274 & 10 & $\mathrm{~N}$ & 1 & 7684 & -34.28 & 7854 & 4875 & -38.21 & 4943 & 7.499E-05 & $7.498 \mathrm{E}-05$ & $0.01 \%$ \\
\hline $03 / 05 / 08$ & 38.274 & 20 & $\mathrm{~N}$ & 1 & 7702 & -34.25 & 7873 & 4576 & -38.79 & 4636 & 8.870E-05 & $8.870 \mathrm{E}-05$ & $0.01 \%$ \\
\hline $03 / 03 / 08$ & 38.274 & 25 & $\mathrm{~N}$ & 1 & 7596 & -31.10 & 7841 & 4378 & -35.82 & 4459 & 9.677E-05 & $9.674 \mathrm{E}-05$ & $0.03 \%$ \\
\hline $03 / 03 / 08$ & 38.274 & 30 & $\mathrm{~N}$ & 1 & 7542 & -30.52 & 7803 & 4195 & -35.60 & 4275 & $1.058 \mathrm{E}-04$ & $1.058 \mathrm{E}-04$ & $0.01 \%$ \\
\hline $01 / 26 / 08$ & Meth. & 20 & $\mathrm{~N}$ & 1 & 7741 & -25.25 & 8246 & 6979 & -26.17 & 7386 & 1.409E-05 & 1.412E-05 & $0.16 \%$ \\
\hline $01 / 26 / 08$ & Meth. & 20 & $\mathrm{~N}$ & 1 & 7752 & -25.41 & 8249 & 6981 & -26.34 & 7381 & 1.424E-05 & $1.426 \mathrm{E}-05$ & $0.11 \%$ \\
\hline $01 / 27 / 08$ & Meth. & 20 & $\mathrm{~N}$ & 1 & 7779 & -26.14 & 8234 & 7004 & -27.07 & 7370 & 1.423E-05 & $1.424 \mathrm{E}-05$ & $0.10 \%$ \\
\hline $04 / 30 / 08$ & Meth. & 20 & $Y$ & 11 & 7569 & -29.74 & 7857 & 6838 & -30.60 & 7073 & 1.412E-05 & 1.411E-05 & $0.07 \%$ \\
\hline $04 / 30 / 08$ & Meth. & 20 & $Y$ & 11 & 7573 & -29.76 & 7860 & 6833 & -30.64 & 7066 & $1.430 \mathrm{E}-05$ & 1.429E-05 & $0.04 \%$ \\
\hline $05 / 13 / 08$ & Meth. & 20 & $Y$ & 11 & 7846 & -30.50 & 8118 & 7068 & -31.40 & 7288 & $1.404 \mathrm{E}-05$ & 1.404E-05 & $0.03 \%$ \\
\hline
\end{tabular}

Table D-1 Loaded and Unloaded Q values 



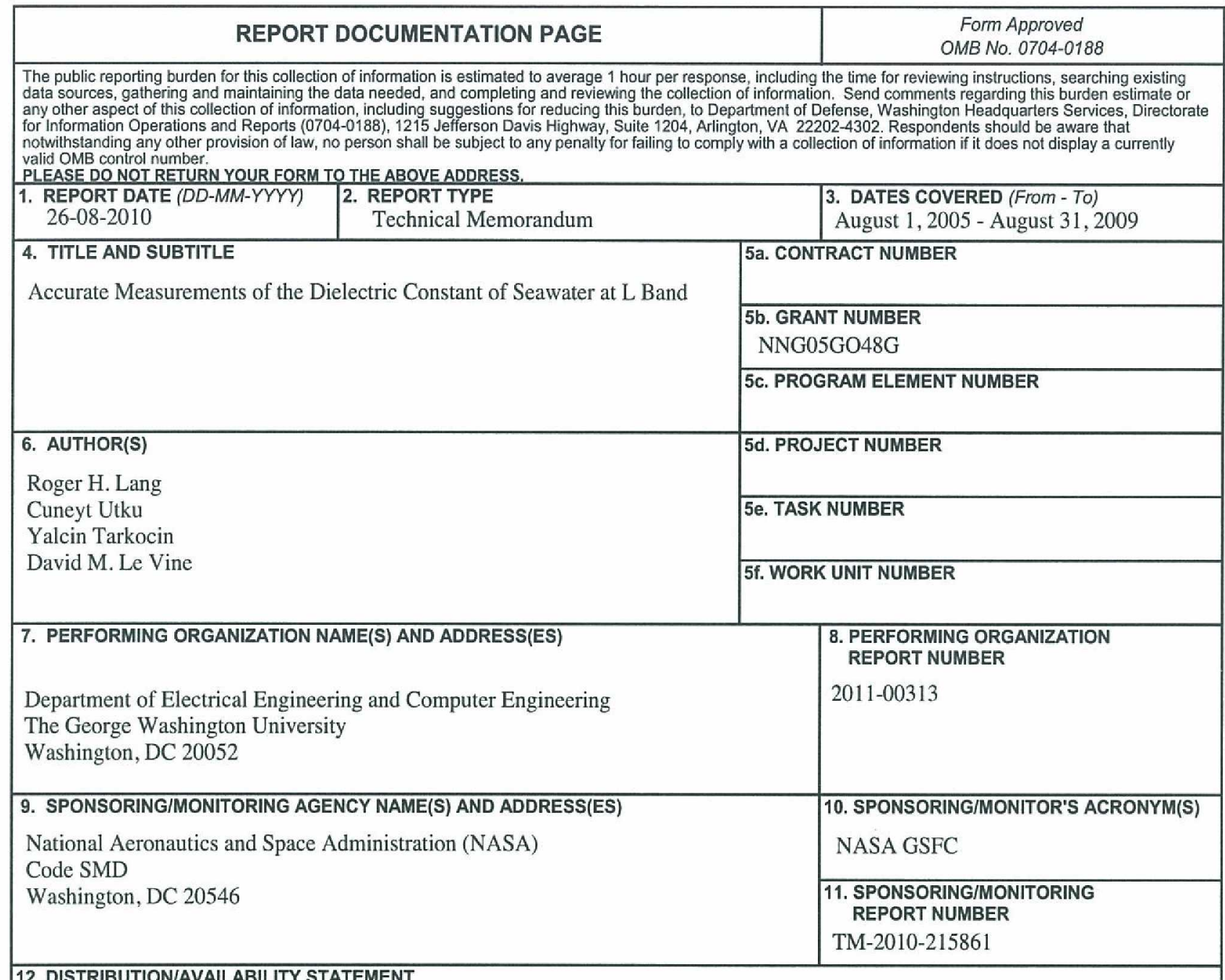

\section{DISTRIBUTION/AVAILABILITY STATEMENT}

Unclassified-Unlimited, Subject Category: 48

Report available from the NASA Center for Aerospace Information, 7115 Standard Drive, Hanover, MD 21076. (443)757-5802

\section{SUPPLEMENTARY NOTES}

\section{ABSTRACT}

This report describes measurements of the dielectric constant of seawater at a frequency of $1.413 \mathrm{GHz}$ that is at the center of the L-Band radiometric protected frequency spectrum. Aquarius will be sensing the sea surface salinity from space in this band. The objective of the project is to refine the model function for the dielectric constant as a function of salinity and temperature so that remote sensing measurements can be made with the accuracy needed to meet the measurement goals ( 0.2 psu) of the Aquarius mission. The measurements were made, using a microwave cavity operated in the transmission configuration. The cavity's temperature was accurately regulated to $0.02^{\circ} \mathrm{C}$ by immersing it in a temperature controlled bath of distilled water and ethanol glycol. Seawater had been purchased from Ocean Scientific International Limited (OSIL) at salinities of 30, 35 and 38 psu. Measurements of these seawater samples were then made over a range of temperatures, from $10^{\circ} \mathrm{C}$ to $35^{\circ} \mathrm{C}$ in $5^{\circ} \mathrm{C}$ intervals. Repeated measurements were made at each temperature and salinity. Mean values and standard deviations were then computed. Total error budgets indicated that the real and imaginary parts of the dielectric constant had a relative accuracy of about $1 \%$. 15. SUBJECT TERMS

Oceanography, Aquarius, L Band, salinity, sampling

\begin{tabular}{|l|l|l|l|l|l|}
\hline \multicolumn{2}{|l|}{ 16. SECURITY CLASSIFICATION OF: } & $\begin{array}{l}\text { 17. LIMITATION OF } \\
\text { ABSTRACT }\end{array}$ & $\begin{array}{l}\text { 18. NUMBER } \\
\text { OF } \\
\text { PAGES }\end{array}$ & $\begin{array}{l}\text { 19b. NAME OF RESPONSIBLE PERSON } \\
\text { D. M. Le Vine }\end{array}$ \\
\cline { 1 - 2 } $\begin{array}{l}\text { a. REPORT } \\
\text { U }\end{array}$ & $\begin{array}{l}\text { b. ABSTRACT } \\
\end{array}$ & $\begin{array}{l}\text { c. THIS PAGE } \\
\text { UU }\end{array}$ & $\begin{array}{l}\text { 19b. TELEPHONE NUMBER (Include area code) } \\
\text { (301) } 614-5640\end{array}$ \\
\hline
\end{tabular}



